\title{
Planck intermediate results. XXII. Frequency dependence of thermal emission from Galactic dust in intensity and polarization $\star$
}

\author{
Planck Collaboration: P. A. R. Ade ${ }^{75}$, M. I. R. Alves ${ }^{51}$, G. Aniano ${ }^{51}$, C. Armitage-Caplan ${ }^{78}$, M. Arnaud ${ }^{64}$, \\ F. Atrio-Barandela ${ }^{17}$, J. Aumont ${ }^{51}$, C. Baccigalupi ${ }^{74}$, A. J. Banday ${ }^{80,10}$, R. B. Barreiro ${ }^{58}$, E. Battaner ${ }^{82,83}$, K. Benabed ${ }^{52,79}$, \\ A. Benoit-Lévy $23,52,79$, J.-P. Bernard ${ }^{80,10}$, M. Bersanelli3 ${ }^{31,44}$, P. Bielewicz ${ }^{80,10,74}$, J. J. Bock ${ }^{59,11}$, J. R. Bond ${ }^{8}$, J. Borrill ${ }^{13,76}$, \\ F. R. Bouchet ${ }^{52,79}$, F. Boulanger ${ }^{51}$, C. Burigana ${ }^{43,29}$, J.-F. Cardoso ${ }^{65,1,52}$, A. Catalano ${ }^{66,63}$, A. Chamballu ${ }^{64,14,51}$, \\ H. C. Chiang ${ }^{25,6}$, L. P. L. Colombo 22,59 , C. Combet ${ }^{66}$, F. Couchot ${ }^{62}$, A. Coulais ${ }^{63}$, B. P. Crill ${ }^{59,72}$, A. Curto ${ }^{5,58}$, F. Cuttaia ${ }^{43}$, \\ L. Danese ${ }^{74}$, R. D. Davies ${ }^{60}$, R. J. Davis ${ }^{60}$, P. de Bernardis ${ }^{30}$, G. de Zotti ${ }^{40,74}$, J. Delabrouille ${ }^{1}$, F.-X. Désert ${ }^{48}$, \\ C. Dickinson ${ }^{60}$, J. M. Diego ${ }^{58}$, S. Donzelli ${ }^{44}$, O. Doré ${ }^{59,11}$, M. Douspis ${ }^{51}$, J. Dunkley ${ }^{78}$, X. Dupac ${ }^{37}$, T. A. Enßlin ${ }^{69}$,
} H. K. Eriksen ${ }^{55}$, E. Falgarone ${ }^{63}$, F. Finelli ${ }^{43,45}$, O. Forni ${ }^{80,10}$, M. Frailis ${ }^{42}$, A. A. Fraisse ${ }^{25}$, E. Franceschi ${ }^{43}$, S. Galeotta ${ }^{42}$, K. Ganga ${ }^{1}$, T. Ghosh ${ }^{51, \star \star}$, M. Giard ${ }^{80,10}$, J. González-Nuevo ${ }^{58,74}$, K. M. Górski ${ }^{59,84}$, A. Gregorio ${ }^{32,42,47}$, A. Gruppuso ${ }^{43}$, V. Guillet ${ }^{51}$, F. K. Hansen ${ }^{55}$, D. L. Harrison ${ }^{54,61}$, G. Helou ${ }^{11}$, C. Hernández-Monteagudo ${ }^{12,69}$, S. R. Hildebrandt ${ }^{11}$, E. Hivon ${ }^{52,79}$, M. Hobson ${ }^{5}$, W. A. Holmes ${ }^{59}$, A. Hornstrup ${ }^{15}$, A. H. Jaffe ${ }^{49}$, T. R. Jaffe ${ }^{80,10}$, W. C. Jones ${ }^{25}$, E. Keihänen ${ }^{24}$, R. Keskitalo ${ }^{13}$, T. S. Kisner ${ }^{68}$, R. Kneiss ${ }^{36,7}$, J. Knoche ${ }^{69}$, M. Kunz ${ }^{16,51,2}$, H. Kurki-Suonio ${ }^{24,39}$, G. Lagache ${ }^{51}$, J.-M. Lamarre ${ }^{63}$, A. Lasenby ${ }^{5,61}$, C. R. Lawrence ${ }^{59}$, J. P. Leahy ${ }^{60}$, R. Leonardi ${ }^{37}$, F. Levrier ${ }^{63}$, M. Liguori2 ${ }^{28}$, P. B. Lilje ${ }^{55}$, M. Linden-Vørnle ${ }^{15}$, M. López-Caniego ${ }^{58}$, P. M. Lubin ${ }^{26}$, J. F. Macías-Pérez ${ }^{66}$, B. Maffei ${ }^{60}$, A. M. Magalhães ${ }^{57}$, D. Maino ${ }^{31,44}$, N. Mandolesi' ${ }^{43,4,29}$, M. Maris ${ }^{42}$, D. J. Marshall ${ }^{64}$, P. G. Martin ${ }^{8}$, E. Martínez-González ${ }^{58}$, S. Masi ${ }^{30}$, S. Matarrese ${ }^{28}$, P. Mazzotta ${ }^{33}$, A. Melchiorri ${ }^{30,46}$, L. Mendes ${ }^{37}$, A. Mennella ${ }^{31,44}$, M. Migliaccio ${ }^{54,61}$, M.-A. Miville-Deschênes ${ }^{51,8}$, A. Moneti ${ }^{52}$, L. Montier ${ }^{80,10}$, G. Morgante ${ }^{43}$, D. Mortlock ${ }^{49}$, D. Munshi' ${ }^{75}$, J. A. Murphy ${ }^{70}$,

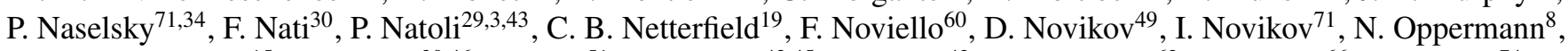
C. A. Oxborrow ${ }^{15}$, L. Pagano ${ }^{30,46}$, F. Pajot ${ }^{51}$, D. Paoletti ${ }^{43,45}$, F. Pasian ${ }^{42}$, O. Perdereau ${ }^{62}$, L. Perotto ${ }^{66}$, F. Perrotta ${ }^{74}$, F. Piacentini ${ }^{30}$, D. Pietrobon ${ }^{59}$, S. Plaszczynski ${ }^{62}$, E. Pointecouteau ${ }^{80,10}$, G. Polenta ${ }^{3,41}$, L. Popa ${ }^{53}$, G. W. Pratt ${ }^{64}$, J. P. Rachen ${ }^{20,69}$, W. T. Reach ${ }^{81}$, M. Reinecke ${ }^{69}$, M. Remazeilles ${ }^{60,51,1}$, C. Renault ${ }^{66}$, S. Ricciardi ${ }^{43}$, T. Riller ${ }^{69}$, I. Ristorcelli ${ }^{80,10}$, G. Rocha ${ }^{59,11}$, C. Rosset $^{1}$, G. Roudier ${ }^{1,63,59}$, J. A. Rubiño-Martín ${ }^{56,35}$, B. Rusholme ${ }^{50}$, E. Salerno ${ }^{9}$, M. Sandri ${ }^{43}$, G. Savini ${ }^{73}$, D. Scott ${ }^{21}$, L. D. Spencer ${ }^{75}$, V. Stolyarov ${ }^{5,61,77}$, R. Stompor ${ }^{1}$, R. Sudiwala ${ }^{75}$, D. Sutton ${ }^{54,61}$, A.-S. Suur-Uski ${ }^{24,39}$, J.-F. Sygnet ${ }^{52}$, J. A. Tauber ${ }^{38}$, L. Terenzi ${ }^{43}$, L. Toffolatti ${ }^{18,58}$, M. Tomasi ${ }^{31,44}$, M. Tristram ${ }^{62}$, M. Tucci ${ }^{16,62}$, L. Valenziano ${ }^{43}$, J. Valiviita ${ }^{24,39}$, B. Van Tent ${ }^{67}$, P. Vielva ${ }^{58}$, F. Villa ${ }^{43}$, B. D. Wandelt ${ }^{52,79,27}$, A. Zacchei ${ }^{42}$, and A. Zonca ${ }^{26}$

(Affiliations can be found after the references)

Received 28 April 2014 / Accepted 9 December 2014

\section{ABSTRACT}

Planck has mapped the intensity and polarization of the sky at microwave frequencies with unprecedented sensitivity. We use these data to characterize the frequency dependence of dust emission. We make use of the Planck $353 \mathrm{GHz} I, Q$, and $U$ Stokes maps as dust templates, and cross-correlate them with the Planck and WMAP data at 12 frequencies from 23 to $353 \mathrm{GHz}$, over circular patches with $10^{\circ}$ radius. The cross-correlation analysis is performed for both intensity and polarization data in a consistent manner. The results are corrected for the chance correlation between the templates and the anisotropies of the cosmic microwave background. We use a mask that focuses our analysis on the diffuse interstellar medium at intermediate Galactic latitudes. We determine the spectral indices of dust emission in intensity and polarization between 100 and $353 \mathrm{GHz}$, for each sky patch. Both indices are found to be remarkably constant over the sky. The mean values, $1.59 \pm 0.02$ for polarization and $1.51 \pm 0.01$ for intensity, for a mean dust temperature of $19.6 \mathrm{~K}$, are close, but significantly different $(3.6 \sigma)$. We determine the mean spectral energy distribution (SED) of the microwave emission, correlated with the $353 \mathrm{GHz}$ dust templates, by averaging the results of the correlation over all sky patches. We find that the mean SED increases for decreasing frequencies at $v<60 \mathrm{GHz}$ for both intensity and polarization. The rise of the polarization SED towards low frequencies may be accounted for by a synchrotron component correlated with dust, with no need for any polarization of the anomalous microwave emission. We use a spectral model to separate the synchrotron and dust polarization and to characterize the spectral dependence of the dust polarization fraction. The polarization fraction $(p)$ of the dust emission decreases by $(21 \pm 6) \%$ from 353 to $70 \mathrm{GHz}$. We discuss this result within the context of existing dust models. The decrease in $p$ could indicate differences in polarization efficiency among components of interstellar dust (e.g., carbon versus silicate grains). Our observational results provide inputs to quantify and optimize the separation between Galactic and cosmological polarization.

Key words. polarization - ISM: general - Galaxy: general - radiation mechanisms: general - submillimeter: ISM - infrared: ISM

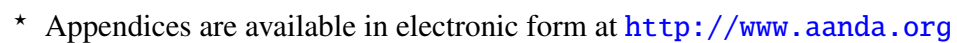

$\star \star$ Corresponding author: T. Ghosh, e-mail: tuhin.ghosh@ias.u-psud.fr
} 


\section{Introduction}

Planck $^{1}$ (Tauber et al. 2010; Planck Collaboration I 2011) has mapped the polarization of the sky emission in seven channels at microwave frequencies from 30 to $353 \mathrm{GHz}$. The data open new opportunities for investigating the astrophysics of Galactic polarization. In this paper, we use these data to characterize the frequency dependence of dust polarization from the diffuse interstellar medium (ISM).

At microwave frequencies, dust emission components include the long-wavelength tail of thermal dust emission (Draine \& Li 2007; Meny et al. 2007; Compiègne et al. 2011; Jones et al. 2013), the anomalous microwave emission (AME, Kogut et al. 1996; Leitch et al. 1997; de Oliveira-Costa et al. 1999; Banday et al. 2003; Lagache 2003; Davies et al. 2006; Dobler \& Finkbeiner 2008; Miville-Deschênes et al. 2008; Ysard et al. 2010; Planck Collaboration XX 2011), and possibly dipolar magnetic emission of ferromagnetic particles (Draine \& Lazarian 1999; Draine \& Hensley 2013).

Thermal dust emission is known to be polarized, but to a different degree for each dust component, owing to differences in the shape and alignment efficiency of grains (Hildebrand et al. 1999; Martin 2007; Draine \& Fraisse 2009). The polarization of the $9.7 \mu \mathrm{m}$ absorption feature from silicates is direct evidence that silicate grains are aligned (Smith et al. 2000). The lack of polarization of the $3.4 \mu \mathrm{m}$ absorption feature from aliphatic hydrocarbons (along lines of sight towards the Galactic centre with strong polarization in the $9.7 \mu \mathrm{m}$ silicate absorption) indicates that dust comprises carbon grains that are much less efficient at producing interstellar polarization than silicates (Chiar et al. 2006). Observational signatures of these differences in polarization efficiency among components of interstellar dust are expected to be found in the polarization fraction $(p)$ of the far infrared (FIR) and sub-mm dust emission. Spectral variations of polarization fraction have been reported from observations of star-forming molecular clouds (Hildebrand et al. 1999; Vaillancourt 2002; Vaillancourt et al. 2008; Vaillancourt $\&$ Matthews 2012). However, these data cannot be unambiguously interpreted as differences in the intrinsic polarization of dust components (Vaillancourt 2002); they can also be interpreted as correlated changes in grain temperature and alignment efficiency across the clouds. The sensitivity of Planck to lowbrightness extended-emission allows us to carry out this investigation for the diffuse ISM, where the heating and alignment efficiency of grains are far more homogeneous than in star-forming regions.

AME is widely interpreted as dipole radiation from small carbon dust particles. This interpretation, first proposed by Erickson (1957) and modelled by Draine \& Lazarian (1998), has been developed into detailed models (Ali-Haïmoud et al. 2009; Silsbee et al. 2011; Hoang et al. 2011) that provide a good spectral fit to the data (Planck Collaboration XX 2011; Planck Collaboration Int. XV 2014). The intrinsic polarization of this emission must be low, owing to the weakness or absence of polarization of the $220 \mathrm{~nm}$ bump in the UV extinction curve (Wolff et al. 1997), which is evidence of the poor alignment of small carbon particles. The polarization fraction of the AME could be up to a few

\footnotetext{
1 Planck (http://www.esa.int/Planck) is a project of the European Space Agency (ESA) with instruments provided by two scientific consortia funded by ESA member states (in particular the lead countries France and Italy), with contributions from NASA (USA) and telescope reflectors provided by a collaboration between ESA and a scientific consortium led and funded by Denmark.
}

percentage (Lazarian \& Draine 2000; Hoang et al. 2013). The Wilkinson Microwave Anisotropy Probe (WMAP) data have been used to search for polarization in a few sources with bright AME, for example the $\rho$ Ophichus and Perseus molecular clouds (Dickinson et al. 2011; López-Caraballo et al. 2011), yielding upper limits in the range of $1.5 \%$ to a few percent on polarization fraction (Rubiño-Martín et al. 2012).

Magnetic dipolar emission (MDE) from magnetic grains was first proposed by Draine \& Lazarian (1999) as a possible interpretation of the AME. Draine \& Hensley (2013) have recently revived this idea with a new model where the MDE could be a significant component of dust emission at frequencies from 50 to a few hundred $\mathrm{GHz}$ (Planck Collaboration Int. XIV 2014) and Planck Collaboration Int. XVII (2014), relevant to cosmic microwave background (CMB) studies. Recently, Liu et al. (2014) have argued that MDE may be contributing to the microwave emission of Galactic radio loops, in particular Loop I. This hypothesis may be tested with the Planck polarization observations. The polarization fraction of MDE is expected to be high for magnetic grains. If the magnetic particles are inclusions within silicates, the polarization directions of the dipolar magnetic and electric emissions are orthogonal. In this case the models predict a significant decrease in the polarization fraction of dust emission at frequencies below $350 \mathrm{GHz}$.

WMAP provided the first all-sky survey of microwave polarization. Galactic polarization was detected on large angular scales at all frequencies from 23 to $94 \mathrm{GHz}$. The data have been shown to be consistent with a combination of synchrotron and dust contributions (Kogut et al. 2007; Page et al. 2007; MivilleDeschênes et al. 2008; Macellari et al. 2011), but they do not constrain the spectral dependence of dust polarization.

The spectral dependence of the dust emission at Planck frequencies has been determined in the Galactic plane and at high Galactic latitudes by Planck Collaboration Int. XIV (2014) and Planck Collaboration Int. XVII (2014). In this paper, we use the high signal-to-noise $353 \mathrm{GHz}$ Planck Stokes $I, Q, U$ maps as templates to characterize the spectral dependence of dust emission in both intensity and polarization. Our analysis also includes the separation of dust emission from CMB anisotropies. We extract the dust-correlated emission in intensity $(I)$ and polarization $(P)$ by cross-correlating the $353 \mathrm{GHz}$ maps with both the Planck and WMAP data. For the intensity, we also use the $\mathrm{H} \alpha$ and $408 \mathrm{MHz}$ maps as templates of the free-free and synchrotron emission. The $P$ and $I$ spectra are compared and discussed in light of the present understanding and questions about microwave dust emission components introduced in Planck Collaboration Int. XVII (2014). We aim to characterize the spectral shape and the relative amplitude of Galactic emission components in polarization. In doing so we test theoretical predictions about the nature of the dust emission in intensity and polarization. We also provide information that is key to designing and optimizing the separation of the polarized CMB signal from the polarized Galactic dust emission.

The paper is organised as follows. In Sect. 2, we introduce the data sets used in this paper. Our methodology for the data analysis is described in the following three sections. We define the part of the sky we analyse in Sect. 3. We describe how we apply the cross-correlation (CC) analysis to the intensity and polarization data in Sect. 4. Section 5 explains the separation of the dust and CMB emission after data correlation. The scientific results are presented in Sects. 6 and 7 for intensity, and Sects. 8 and 9 for polarization. The dust SEDs, $I$ and $P$, are compared and discussed with relation to models of dust emission in Sect. 10. Section 11 summarizes the main results of our 
Planck Collaboration: Frequency dependence of thermal emission from Galactic dust in intensity and polarization

Table 1. Summary of Planck, WMAP and ancillary data used in this paper for both intensity and polarization.

\begin{tabular}{|c|c|c|c|}
\hline Telescope/survey & $\begin{array}{c}\text { Frequency } \\
\quad[\mathrm{GHz}]\end{array}$ & $\begin{array}{l}\text { Resolution } \\
\text { [arcmin] }\end{array}$ & Reference \\
\hline Haslam . . . . . . & 0.408 & 60 & Haslam et al. (1982) \\
\hline WMAP 9-year . . . & 23 & 48.42 & Bennett et al. (2013) \\
\hline Planck......... . & 28.4 & 32.23 & Planck Collaboration I (2014) \\
\hline WMAP 9-year . . . & 33 & 37.44 & Bennett et al. (2013) \\
\hline WMAP 9-year . . . & 41 & 28.62 & Bennett et al. (2013) \\
\hline Planck. . . . . . . . & 44.1 & 27.01 & Planck Collaboration I (2014) \\
\hline WMAP 9-year . . . & 61 & 19.56 & Bennett et al. (2013) \\
\hline Planck. . . . . . . . & 70.4 & 13.25 & Planck Collaboration I (2014) \\
\hline WMAP 9-year . . . & 94 & 12.30 & Bennett et al. (2013) \\
\hline Planck... . . . . . . & 100 & 9.65 & Planck Collaboration I (2014) \\
\hline Planck... . . . . . & 143 & 7.25 & Planck Collaboration I (2014) \\
\hline Planck. . . . . . . . & 217 & 4.99 & Planck Collaboration I (2014) \\
\hline Planck. . . . . . . . & 353 & 4.82 & Planck Collaboration I (2014) \\
\hline Planck. . . . . . . . & 545 & 4.68 & Planck Collaboration I (2014) \\
\hline Planck... . . . . . & 857 & 4.32 & Planck Collaboration I (2014) \\
\hline DIRBE $\ldots \ldots \ldots$ & 3000 & 50 & Hauser et al. (1998) \\
\hline $\mathrm{H} \alpha \ldots \ldots \ldots$ & & 60 & Dickinson et al. (2003) \\
\hline LAB HI $\ldots \ldots \ldots$ & & 36 & Kalberla et al. (2005) \\
\hline
\end{tabular}

work. We detail the derivation of the correlation coefficients in Appendix A. Appendix B describes the Monte Carlo simulations we have performed to show that our data analysis is unbiased. Appendix $\mathrm{C}$ describes the dependence of the dust $I$ SED on the correction of the $\mathrm{H} \alpha$ map, used as template of the free-free emission, for dust extinction and scattering. The power spectra of the maps used as templates of dust, free-free and synchrotron emission are presented in Appendix D for a set of Galactic masks.

\section{Data sets used}

Here we discuss the Planck, WMAP, and ancillary data used in the paper and listed in Table 1.

\subsection{Planck data}

\subsubsection{Sky maps}

Planck is the third generation space mission to characterize the anisotropies of the CMB. It observed the sky in seven frequency bands from 30 to $353 \mathrm{GHz}$ for polarization, and in two additional bands at 545 and $857 \mathrm{GHz}$ for intensity, with an angular resolution from $31^{\prime}$ to $5^{\prime}$ (Planck Collaboration I 2014). The in-flight performance of the two focal plane instruments, the HFI (High Frequency Instrument) and the LFI (Low Frequency Instrument), are given in Planck HFI Core Team (2011) and Mennella et al. (2011), respectively. The data processing and calibration of the HFI and LFI data used here are described in Planck Collaboration VIII (2014) and Planck Collaboration II (2014), respectively. The data processing specific to polarization is given in Planck Collaboration VI (2014) and Planck Collaboration III (2014).

For intensity, we use the full Planck mission (five full-sky surveys for HFI and eight full-sky surveys for LFI) data sets between 30 and $857 \mathrm{GHz}$. The LFI and HFI frequency maps are provided in HEALPix ${ }^{2}$ format (Górski et al. 2005) with resolution parameters $N_{\text {side }}=1024$ and 2048, respectively. The Planck sky maps between 30 and $353 \mathrm{GHz}$ are calibrated in CMB temperature units, $\mathrm{K}_{\mathrm{CMB}}$, so that the $\mathrm{CMB}$ anisotropies have a constant spectrum across frequencies. The two high frequency maps

\footnotetext{
2 http://healpix.jpl.nasa.gov
}

of Planck, 545 and $857 \mathrm{GHz}$, are expressed in $\mathrm{MJy} \mathrm{sr}^{-1}$, calibrated for a power-law spectrum with a spectral index of -1 , following the IRAS convention. We use Planck maps with the zodiacal light emission (ZLE) subtracted (Planck Collaboration XIV 2014) at frequencies $v \geq 353 \mathrm{GHz}$, but maps not corrected for ZLE at lower frequencies because the extrapolation of the ZLE model is uncertain at microwave frequencies. Further it has not been estimated at frequencies smaller than $100 \mathrm{GHz}$. We do not correct for the zero offset, nor for the residual dipole identified by Planck Collaboration XI (2014) at HFI frequencies because it is not necessary for our analysis based on local correlations of data sets.

For polarization, we use the same full Planck mission data sets, as used for intensity, between 30 and $353 \mathrm{GHz}$. The Planck polarization that we use in this have been generated in exactly the same manner as the data publicly released in March 2013, described in Planck Collaboration I (2014) and associated papers. Note, however, that the publicly available data includes data include only temperature maps based on the first two surveys. Planck Collaboration XVI (2014) shows the very good consistency of cosmological models derived from intensity only with polarization data at small scale scales (high CMB multipoles). However, as detailed in Planck Collaboration VI (2014, see their Fig. 27), the 2013 polarization data are known to be affected by systematic effects at low multipoles which were not yet fully corrected, and thus these data were not used for cosmology. In this paper, we use the latest Planck polarization maps (internal data release "DX11d"), which are corrected from known systematics. The full mission maps for intensity as well as for polarization will be described and made publicly available in early 2015.

\subsubsection{Systematic effects in polarization}

Current Planck polarization data are contaminated by a small amount of leakage from intensity to polarization, mainly due to bandpass mismatch (BPM) and calibration mismatch between detectors (Planck Collaboration Int. XIX 2015; Planck Collaboration VI 2014; Planck Collaboration III 2014). The BPM results from slight differences in the spectral response to Galactic emission of the polarization sensitive bolometers (PSB; Planck Collaboration VI 2014). In addition, the signal 
differences leak into polarization. The calibration uncertainties translate into a small mismatch in the response of the detectors, which produces a signal leakage from intensity to polarization. As the microwave sky is dominated by the large scale emission from the Galaxy and the CMB dipole, systematics affect the polarization maps mainly on large angular scales. We were only able to correct the maps for leakage of Galactic emission due to bandpass mismatch.

The observed Stokes $Q_{v}^{\text {obs }}$ and $U_{v}^{\text {obs }}$ maps at a given frequency $v$ can be written as,

$Q_{v}^{\mathrm{obs}}=Q^{\mathrm{c}}+Q_{v}^{\mathrm{G}}+Q_{v}^{\mathrm{n}}+L_{v}(I \rightarrow Q)$,

$U_{v}^{\mathrm{obs}}=U^{\mathrm{c}}+U_{v}^{\mathrm{G}}+U_{v}^{\mathrm{n}}+L_{v}(I \rightarrow U)$,

where the term $L$ corresponds to the BPM leakage map for Galactic emission, offset, and residual dipole. All of them are computed using the coupling coefficient of each detector to the sky emission spectrum together with the actual sky scanning strategy. The superscript $\mathrm{c}$ represents the CMB polarization, $\mathrm{n}$ represents the noise and the index $\mathrm{G}$ incorporates all the Galactic emission components in intensity at Planck frequencies. We restrict our analysis to intermediate Galactic latitudes where the dominant Galactic emission at HFI frequencies is dust emission. The polarized HFI maps we used are corrected for the dust, $\mathrm{CO}$, offset and residual dipole, to a first approximation, using sky measurements of the spectral transmission of each bolometer (Planck Collaboration IX 2014). At LFI frequencies, we correct for BPM coming from the low frequency Galactic components, i.e., the AME, synchrotron and free-free emission (Planck Collaboration III 2014), using sky measurements of the spectral transmission of each bolometer.

To test the results presented in this paper for systematic effects, we use multiple data sets that include the maps made with two independent groups of four PSBs (detector sets "DS1" and "DS2", see Table 3 in Planck Collaboration VI 2014), the halfring maps (using the first or second halves of the data from each stable pointing period, "HR1" and "HR2") and maps made with yearly surveys ("YR1", "YR2", etc.). The HR1 and HR2 maps are useful to assess the impact on our data analysis of the noise and systematic effects on scales smaller than $20^{\prime}$. The YR1 map is a combination of first two surveys $\mathrm{S} 1$ and S2, and YR2 is a combination of surveys S3 and S4, and so on. The maps made with individual sky surveys are useful to quantify the impact of systematic effects on larger angular scales, particularly from beam ellipticity and far sidelobes (Planck Collaboration III 2014; Planck Collaboration VI 2014). For the intensity and polarization HFI data, we use the two yearly maps YR1 and YR2, whereas for LFI data, we use the four yearly maps grouped into odd (YR1+YR3) and even (YR2+YR4) pairs because they share the same scanning strategy.

The different data sets are independent observations of the same sky that capture noise and systematic effects. They provide means to assess the validity and self-consistency of our analysis of the Planck data. The different map combinations highlight different systematic effects on various timescales and across different dimensions.

- Half-ring maps share the same scanning strategy and detectors so they have the same leakage from intensity to polarization. The difference between these two maps shows the noise that is not correlated. The removal of glitches induce some noise correlation between the two half-ring maps that affects the data at all multipoles.
- The differences between two yearly maps is used to check the consistency of the data over the full duration of the Planck mission.

- Detector set maps have the same combination of scans. The difference between detector set maps show all systematic effects associated with specific detectors.

\subsection{WMAP data}

We use the WMAP nine year data (Bennett et al. 2013) from the Legacy Archive for Microwave Background Data Analysis $(\text { LAMBDA })^{3}$ provided in the HEALPix pixelization scheme with a resolution $N_{\text {side }}=512$. WMAP observed the sky in five frequency bands, denoted $K, K a, Q, V$, and $W$, centred at the frequencies $23,33,41,61$, and $94 \mathrm{GHz}$, respectively. WMAP has ten differencing assemblies (DAs), one for both $K$ and $K a$ bands, two for $Q$ band, two for $V$ band, and four for $W$ band. WMAP has frequency-dependent resolution, ranging from $52^{\prime}$ ( $K$ band) to $12^{\prime}$ ( $W$ band). Multiple DAs at each frequency for $Q, V$ and $W$ bands are combined using simple average to generate a single map per frequency band.

\subsection{Ancillary data}

We complement the Planck and WMAP data with several ancillary sky maps. We use the $408 \mathrm{MHz}$ map from Haslam et al. (1982), and H $\alpha$ map from Dickinson et al. (2003, DDD) as tracers of synchrotron and free-free emission, respectively. No dust extinction correction $\left(f_{\mathrm{d}}=0.0\right)$ has been applied to the DDD $\mathrm{H} \alpha$ map, which is expressed in units of Rayleigh (R). For our simulations we use the Leiden/Argentine/Bonn (LAB) survey of Galactic HI column density (Kalberla et al. 2005) as a tracer of dust emission (Planck Collaboration XXIV 2011; Planck Collaboration Int. XVII 2014). Finally, we use the DIRBE $100 \mu \mathrm{m}$ sky map to determine the dust temperature, like in Planck Collaboration XI (2014).

The $408 \mathrm{MHz}$, LAB HI, and DIRBE $100 \mu \mathrm{m}$ data are downloaded from LAMBDA. We use the DIRBE data corrected for ZLE. We project the DIRBE $100 \mu \mathrm{m}$ map on a HEALPix grid at $N_{\text {side }}=512$ with a Gaussian interpolation kernel that reduces the angular resolution to $50^{\prime}$. Both the $408 \mathrm{MHz}$ and the DDD $\mathrm{H} \alpha$ maps are provided at $1^{\circ}$ resolution. The LAB HI survey and DIRBE $100 \mu \mathrm{m}$ data have angular resolutions of $36^{\prime}$ and $50^{\prime}$, respectively.

\section{Global mask}

In the data analysis we use a global mask, shown in Fig. 1, which selects regions of dust emission from the ISM at intermediate Galactic latitudes. We only want to study polarization in regions where thermal dust emission dominates. This means that we need to remove the area around the Galactic plane, where other Galactic contributions are significant, and remove the high latitude regions, where the anisotropies of the cosmic infrared background (CIB) are important with respect to dust emission. The global mask combines thresholds on several sky emission components (in intensity): carbon monoxide (CO) line emission; free-free; synchrotron; the CIB anisotropies; and point sources. We now detail how the global mask is defined.

In the regions of lowest dust column density at high Galactic latitudes, brightness fluctuations from the CIB are significant. To define the CIB mask, we apply a threshold on the ratio between

3 http://lambda.gsfc.nasa.gov 


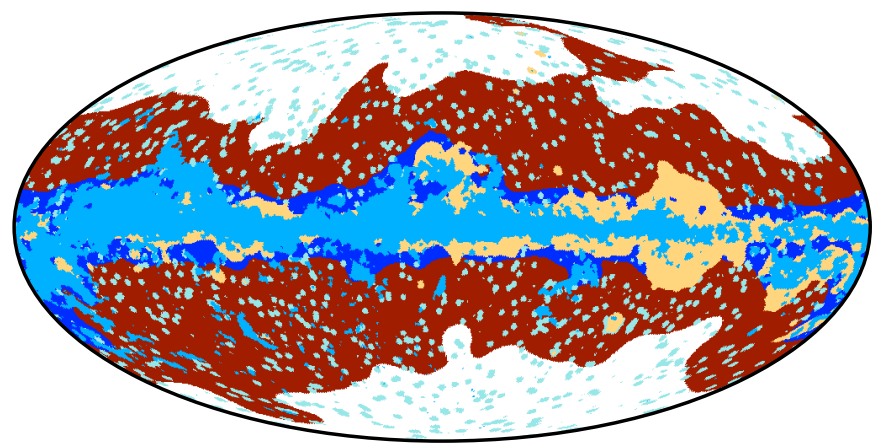

Fig. 1. Global mask used in the cross-correlation (CC) analysis (Mollweide projection in Galactic coordinates). It comprises the CIB mask (white region), the CO mask (light-blue), the free-free mask (beige), the Galactic mask (deep-blue), and the mask of point sources (turquoise). We use the red regions of the sky. We refer readers to Sect. 3 for a detailed description of how the global mask is defined.

the root mean square (rms) of the total Galactic emission and of the $\mathrm{CIB}$ at $353 \mathrm{GHz}$ :

$\left|\frac{\sigma_{\mathrm{G}}}{\sigma_{\mathrm{CIB}}}\right|<9$

where $\sigma_{\mathrm{G}}$ and $\sigma_{\mathrm{CIB}}$ are defined as

$\sigma_{\mathrm{CIB}}^{2}=\sum_{\ell} \frac{2 \ell+1}{4 \pi} C_{\ell}^{\mathrm{CIB}} b_{\ell}^{2} w_{\ell}^{2}$,

$\sigma_{\mathrm{c}}^{2}=\sum_{\ell} \frac{2 \ell+1}{4 \pi} C_{\ell}^{\mathrm{c}} b_{\ell}^{2} w_{\ell}^{2}$,

$\sigma_{\mathrm{G}}^{2}=\sigma^{2}\left(I_{353}\right)-\sigma_{\mathrm{c}}^{2}-\sigma_{\mathrm{CIB}}^{2}$

For this threshold, the CIB contribution to the CC coefficients in Sect. 4.2 is smaller than about $1 \%\left(1 / 9^{2}\right)$ of that of the total Galactic emission at $353 \mathrm{GHz}$. The summation is over the multipole range $15<\ell<300$ (corresponding to an effective range of angular scales from $1^{\circ}$ to $\left.10^{\circ}\right) . C_{\ell}^{\mathrm{CIB}}$ is the best-fit CIB power spectrum at $353 \mathrm{GHz}$ (Planck Collaboration XXX 2014), $C_{\rho}^{\mathrm{c}}$ is the best-fit $\mathrm{CMB}$ power spectrum (Planck Collaboration XV 2014), $I_{353}$ represents the Planck $353 \mathrm{GHz}$ map, $b_{\ell}$ is the beam function and $w_{\ell}$ is the HEALPix pixel window function. We measure the Galactic to CIB emission ratio over patches with $10^{\circ}$ radius centred on HEALPix pixels at a resolution $N_{\text {side }}=32$.

The CO, free-free, and synchrotron emission are more important close to the Galactic plane. The first three $\mathrm{CO}$ line transitions $J=1 \rightarrow 0, J=2 \rightarrow 1$, and $J=3 \rightarrow 2$ at 115, 230, and $345 \mathrm{GHz}$, respectively, are significant emission components in the Planck intensity maps (Planck Collaboration XIII 2014). The CO mask is defined by applying a threshold of $0.5 \mathrm{~K} \mathrm{~km} \mathrm{~s}^{-1}$ on the "Type 2" CO $J=1 \rightarrow 0$, which is extracted using the Planck data between 70 and $353 \mathrm{GHz}$ (Planck Collaboration XIII 2014). The free-free emission is weak compared to the CO line emission at $100 \mathrm{GHz}$ for most molecular clouds. In massive star-forming regions and for the diffuse Galactic plane emission, free-free emission is significant (Planck Collaboration Int. XIV 2014). We take the WMAP maximum entropy method free-free map (Bennett et al. 2013) at $94 \mathrm{GHz}$ and apply a threshold of $10 \mu \mathrm{K}_{\mathrm{RJ}}$ (in Rayleigh-Jeans temperature units) to define the free-free mask. In addition, we use the Galactic mask (CS-CR75) from the Planck component separation results (Planck Collaboration XII 2014) to exclude the synchrotron emission from the Galactic plane and the Galactic "haze"
(Planck Collaboration Int. IX 2013). We also apply the Planck point source mask (Planck Collaboration XV 2014).

Our mask focuses on the part of the sky where dust is the dominant emission component at HFI frequencies. This choice makes the spectral leakage from free-free and CO line emissions to polarization maps negligible. After masking we are left with $39 \%$ of the sky at intermediate Galactic latitudes $\left(10^{\circ}<\right.$ $|b|<60^{\circ}$ ). The same global mask is used for both intensity and polarization correlation analysis to compare results over the same sky.

\section{Cross-correlation method}

We use the CC analysis adopted in many studies (Banday et al. 1996; Gorski et al. 1996; Davies et al. 2006; Page et al. 2007; Ghosh et al. 2012; Planck Collaboration Int. XII 2013) to extract the signal correlated with the $353 \mathrm{GHz}$ template in intensity and polarization. The only underlying assumption is that the spatial structure in the $353 \mathrm{GHz}$ template and in the map under analysis are locally correlated. To reduce this assumption, we apply the $\mathrm{CC}$ analysis locally over patches of sky of $10^{\circ}$ radius (Sect. 4.4). Our choice for the dust template is presented in Sect. 4.1. The methodology is introduced for intensity in Sect. 4.2 and for polarization in Sect. 4.3. The practical implementation of the method is outlined in Sect. 4.4.

\section{1. $353 \mathrm{GHz}$ template}

We perform the $\mathrm{CC}$ analysis locally in the pixel domain using the Planck $353 \mathrm{GHz}$ maps of Stokes parameters as representative internal templates for dust emission in intensity ( $I$ with the ZLE subtracted) and polarization $(Q$ and $U)$. Our choice of a Planck map as a dust template addresses some of the issues plaguing alternative choices. First, unlike the HI map, the $353 \mathrm{GHz}$ map traces the dust in both $\mathrm{HI}$ and $\mathrm{H}_{2}$ gas (Reach et al. 1998; Planck Collaboration XXIV 2011; Planck Collaboration Int. XVII 2014). Second, unlike the full-sky Finkbeiner et al. (1999, hereafter FDS) $94 \mathrm{GHz}$ map, the $353 \mathrm{GHz}$ map does not rely on an extrapolation over a large frequency range, from $100 \mu \mathrm{m}$ to the Planck bands. The main drawback of the $353 \mathrm{GHz}$ template is that it includes CMB and CIB anisotropies. By introducing the global mask, we work with the sky region where the CIB anisotropies are small compared to dust emission. However, the contribution of the CMB to the CC coefficients, most significant at microwave frequencies, needs to be subtracted.

\subsection{Intensity}

\subsubsection{Correlation with the $353 \mathrm{GHz}$ template}

For the intensity data, the CC coefficient $\left(\alpha_{v}^{\mathrm{I}}\right)$ is obtained by minimizing the $\chi_{\mathrm{I}}^{2}$ expression given by,

$\chi_{\mathrm{I}}^{2}=\sum_{k=1}^{N_{\text {pix }}}\left[I_{v}(k)-\left[\alpha_{v}^{\mathrm{I}}\right]_{353}^{1 \mathrm{~T}} I_{353}(k)-a\right]^{2}$,

where $I_{v}$ and $I_{353}$ denote the data and the $353 \mathrm{GHz}$ template maps, respectively. This is a linear fit and the solution is computed analytically. Here the $\mathrm{CC}$ coefficient is a number in $\mathrm{K}_{\mathrm{CMB}}$ $\mathrm{K}_{\mathrm{CMB}}{ }^{-1}$, as both $I_{v}$ and $I_{353}$ are expressed in $\mathrm{K}_{\mathrm{CMB}}$ units. The constant offset, $a$, takes into account the local mean present in the template as well as in the data. The sum is over the unmasked pixels, $k$, within a given sky patch. We are insensitive 
to the residual dipole present at Planck frequencies because we perform local correlation over $10^{\circ}$ radius patches. The index "1T" represents the $353 \mathrm{GHz}$ correlated coefficient at a given frequency $v$ that we obtained using one template only.

The CC coefficient at a given frequency includes the contribution from all the emission components that are correlated with the $353 \mathrm{GHz}$ template (Appendix A). It can be decomposed into the following terms:

$$
\begin{aligned}
{\left[\alpha_{v}^{\mathrm{I}}\right]_{353}^{1 \mathrm{~T}}=} & \alpha^{\mathrm{I}}\left(c_{353}^{1}\right)+\alpha_{v}^{\mathrm{I}}\left(d_{353}\right)+\alpha_{v}^{\mathrm{I}}\left(s_{353}\right) \\
& +\alpha_{v}^{\mathrm{I}}\left(f_{353}\right)+\alpha_{v}^{\mathrm{I}}\left(a_{353}\right)
\end{aligned}
$$

where $c_{353}^{1}, d_{353}, s_{353}, f_{353}$, and $a_{353}$ refer to the CMB, dust, synchrotron, free-free, and AME signals that are correlated with the $353 \mathrm{GHz}$ template, respectively. The $\mathrm{CMB} \mathrm{CC}$ coefficient term is achromatic because Eq. (8) is expressed in $\mathrm{K}_{\mathrm{CMB}}$ units. We neglect the contributions of the three CO lines, point sources, and the CIB anisotropies, since these are subdominant within our global mask (Sect. 3). We also neglect the cross-correlation of the ZLE with the dust template. The chance correlations between the emission components we neglect and the dust template contribute to the statistical uncertainties on the dust SED, but do not bias it. We checked this with Monte Carlo simulations (Appendix B) and repeated our analysis on HFI maps with the ZLE subtracted. The correlation terms of the synchrotron and AME components are negligible at $v \geq 100 \mathrm{GHz}$, as synchrotron and AME both have a steep spectrum that falls off fast at high frequencies. The free-free emission is weak outside the Galactic plane at high frequencies and does not contribute significantly to the $\mathrm{CC}$ coefficients. The synchrotron, AME and free-free terms only become significant at $v<100 \mathrm{GHz}$ inside our global mask.

\subsubsection{Correlation with two and three templates}

To remove $\alpha_{v}^{\mathrm{I}}\left(s_{353}\right)$ and $\alpha_{v}^{\mathrm{I}}\left(f_{353}\right)$ in Eq. (8), we cross-correlate the Planck and WMAP data with either two or three templates (including the dust template). We use the $353 \mathrm{GHz}$ and the $408 \mathrm{MHz}$ maps for the fit with two templates, and add the DDD H $\alpha$ map for the three-template fit. The $\chi_{\mathrm{I}}^{2}$ expressions that we minimize for these two cases are

$$
\begin{aligned}
\chi_{\mathrm{I}}^{2}= & \sum_{k=1}^{N_{\text {pix }}}\left[I_{v}(k)-\left[\alpha_{v}^{\mathrm{I}}\right]_{353}^{2 \mathrm{~T}} I_{353}(k)-\left[\alpha_{v}^{\mathrm{I}}\right]_{0.408}^{2 \mathrm{~T}} I_{0.408}(k)-a\right]^{2}, \\
\chi_{\mathrm{I}}^{2}= & \sum_{k=1}^{N_{\mathrm{pix}}}\left[I_{v}(k)-\left[\alpha_{v}^{\mathrm{I}}\right]_{353}^{3 \mathrm{~T}} I_{353}(k)-\left[\alpha_{v}^{\mathrm{I}}\right]_{0.408}^{3 \mathrm{~T}} I_{0.408}(k)\right. \\
& \left.-\left[\alpha_{v}^{\mathrm{I}}\right]_{\mathrm{H} \alpha}^{3 \mathrm{~T}} I_{\mathrm{H} \alpha}(k)-a\right]^{2},
\end{aligned}
$$

where $I_{v}, I_{353}, I_{0.408}$, and $I_{\mathrm{H} \alpha}$ denote the data at a frequency $v$, the Planck $353 \mathrm{GHz}$, Haslam $408 \mathrm{MHz}$, and DDD H $\alpha$ maps, respectively. For these multiple template fits, the CC coefficients are given by

$$
\begin{aligned}
& {\left[\alpha_{v}^{\mathrm{I}}\right]_{353}^{2 \mathrm{~T}}=\alpha^{\mathrm{I}}\left(c_{353}^{2}\right)+\alpha_{v}^{\mathrm{I}}\left(d_{353}\right)+\alpha_{v}^{\mathrm{I}}\left(f_{353}\right)+\alpha_{v}^{\mathrm{I}}\left(a_{353}\right)} \\
& {\left[\alpha_{v}^{\mathrm{I}}\right]_{353}^{3 \mathrm{~T}}=\alpha^{\mathrm{I}}\left(c_{353}^{3}\right)+\alpha_{v}^{\mathrm{I}}\left(d_{353}\right)+\alpha_{v}^{\mathrm{I}}\left(a_{353}\right) .}
\end{aligned}
$$

The indices " $2 \mathrm{~T}$ " and " $3 \mathrm{~T}$ " are used here to distinguish the CC coefficients for the fit with two and three templates, respectively. The use of additional templates removes the corresponding terms from the right hand side of these equations. Equation (12) is used to derive the mean dust SED in intensity. Equations (8), (11), and (12) may be combined to derive $\alpha_{v}^{\mathrm{I}}\left(s_{353}\right)$ and $\alpha_{v}^{\mathrm{I}}\left(f_{353}\right)$.

\subsection{Polarization}

For the polarization data, we cross-correlate both the Stokes $Q$ and $U 353 \mathrm{GHz}$ templates with the $Q$ and $U$ maps for all Planck and WMAP frequencies. Ideally in CC analysis, the template is free from noise, but the Planck $353 \mathrm{GHz}$ polarization templates do contain noise, which may bias the $\mathrm{CC}$ coefficients. To circumvent this problem, we use two independent $Q$ and $U$ maps made with the two detector sets DS1 and DS2 at $353 \mathrm{GHz}$ as templates (Sect. 2.1.2). The maps made with each of the two detector sets have independent noise and dust BPM. Using two polarization detector sets at $353 \mathrm{GHz}$ with independent noise realizations reduces the noise bias in the determination of the $353 \mathrm{GHz}$ $\mathrm{CC}$ coefficients. We use the detector set maps rather than the half-ring maps because the removal of glitches induces some noise correlation between the two half-ring maps that affects the data at all multipoles (Planck Collaboration VI 2014; Planck Collaboration X 2014).

The polarization CC coefficient $\left(\alpha_{v}^{\mathrm{P}}\right)$ is derived by minimizing the $\chi_{\mathrm{P}}^{2}$ expression given by

$$
\begin{aligned}
\chi_{\mathrm{P}}^{2}= & \sum_{i=1}^{2} \sum_{k=1}^{N_{\text {pix }}}\left[Q_{v}(k)-\left[\alpha_{v}^{\mathrm{P}}\right]_{353}^{1 \mathrm{~T}} Q_{353}^{i}(k)-a\right]^{2} \\
& +\left[U_{v}(k)-\left[\alpha_{v}^{\mathrm{P}}\right]_{353}^{1 \mathrm{~T}} U_{353}^{i}(k)-b\right]^{2},
\end{aligned}
$$

where the index $i$ takes the values 1 and 2, which correspond to the DS1 and DS2 maps at $353 \mathrm{GHz}$. The summation $k$ is over the unmasked pixels within a given sky patch. The constant offsets $a$ and $b$ take into account the local mean present in the template as well as in the data Stokes $Q$ and $U$ maps, respectively. At $353 \mathrm{GHz}$, we cross-correlate the DS1 and DS2 maps of $Q$ and $U$ among themselves, minimizing

$$
\begin{aligned}
\chi_{\mathrm{P}}^{2}= & \sum_{\substack{i=1 \\
i \neq j}}^{2} \sum_{k=1}^{N_{\mathrm{pix}}}\left[Q_{353}^{j}(k)-\left[\alpha_{353}^{\mathrm{P}}\right]_{353}^{1 \mathrm{~T}} Q_{353}^{i}(k)-a\right]^{2} \\
& +\left[U_{353}^{j}(k)-\left[\alpha_{353}^{\mathrm{P}}\right]_{353}^{1 \mathrm{~T}} U_{353}^{i}(k)-b\right]^{2} .
\end{aligned}
$$

The CC coefficients, $\alpha_{v}^{\mathrm{P}}$, comprise the contributions of CMB, dust, synchrotron and possibly AME polarization. The free-free polarization is expected to be negligible theoretically (Rybicki \& Lightman 1979) and has been constrained to a few percent observationally (Macellari et al. 2011). The polarization decomposition is given by

$\left[\alpha_{v}^{\mathrm{P}}\right]_{353}^{1 \mathrm{~T}}=\alpha^{\mathrm{P}}\left(c_{353}^{1}\right)+\alpha_{v}^{\mathrm{P}}\left(d_{353}\right)+\alpha_{v}^{\mathrm{P}}\left(s_{353}\right)+\alpha_{v}^{\mathrm{P}}\left(a_{353}\right)$.

The polarized CMB CC coefficient, $\alpha^{\mathrm{P}}\left(c_{353}^{1}\right)$, is achromatic because Eq. (15) is expressed in $\mathrm{K}_{\mathrm{CMB}}$ units. Unlike for intensity, due to the absence of any polarized synchrotron template free from Faraday rotation (Gardner \& Whiteoak 1966), we cannot perform a fit with two templates to remove $\alpha_{v}^{\mathrm{P}}\left(s_{353}\right)$ in Eq. (15).

We have performed Monte Carlo simulations at the HFI frequencies in order to estimate the uncertainty on the CC coefficient induced by the noise and other Galactic emission present in the data (see Appendix B).

\subsection{Implementation}

Here we describe how we implement the CC method. The Planck, WMAP, HI and DIRBE sky maps are smoothed to a 
common resolution of $1^{\circ}$, taking into account the effective beam response of each map, and reduced to a HEALPix resolution $N_{\text {side }}=128$. For the Planck and WMAP maps, we use the effective beams defined in multipole space that are provided in the Planck Legacy Archive ${ }^{4}$ (PLA) and LAMBDA website (Planck Collaboration VII 2014; Planck Collaboration IV 2014; Bennett et al. 2013). The Gaussian approximation of the average beam widths for Planck and WMAP maps are quoted in Table 1. For the HI and DIRBE maps, we also use Gaussian beams with the widths given in Table 1. For the polarization data, we use the "ismoothing" routine of HEALPix that decomposes the $Q$ and $U$ maps into $E$ and $B a_{\ell m} \mathrm{~s}$, applies Gaussian smoothing of $1^{\circ}$ in harmonic space (after deconvolving the effective azimuthally symmetric beam response for each map), and transforms the smoothed $E$ and $B a_{\ell m}$ s back into $Q$ and $U$ maps at $N_{\text {side }}=128$ resolution.

We divide the intermediate Galactic latitudes into sky patches with $10^{\circ}$ radius centred on HEALPix pixels for $N_{\text {side }}=$ 8 . For a much smaller radius we would have too few independent sky pixels within a given sky patch to measure the mean dust SED. For a much larger radius we would have too few sky patches to estimate the statistical uncertainty on the computation of the mean dust SED. Each sky patch contains roughly 1500 pixels at $N_{\text {side }}=128$ resolution. We only consider 400 sky patches $\left(N_{\text {bins }}\right)$, which have 500 or more unmasked pixels. We then cross-correlate the $353 \mathrm{GHz}$ Planck internal template with the WMAP and Planck maps between 23 and $353 \mathrm{GHz}$, locally in each sky patch to extract the $353 \mathrm{GHz}$ correlated emission in intensity, along with its polarization counterpart. The sky patches used are not strictly independent. Each sky pixel is part of a few sky patches, which is required to sample properly the spatial variations of the $\mathrm{CC}$ coefficients. The mean number of times each pixel is used in CC coefficients $\left(N_{\text {visit }}\right)$ is estimated with the following formula:

$N_{\text {visit }}=\frac{N_{\text {bins }} \times\left\langle N_{\text {pixels }}\right\rangle}{0.39 \times N_{\text {total }}} \sim 5$,

where $N_{\text {total }}=12 \times N_{\text {side }}^{2}$ is the total number of pixels at $1^{\circ}$ resolution, 0.39 is the fraction of the sky used in our analysis and $\left\langle N_{\text {pixels }}\right\rangle=1000$ is the average number of pixels per sky patch after masking.

\section{Component separation methodology}

At the highest frequencies $(v \geq 100 \mathrm{GHz})$ within our mask, the two main contributors to the CC coefficient are the CMB and dust emission. In this section, we detail how we separate them and estimate the spectral index of the dust emission $\left(\beta_{\mathrm{d}}\right)$ in intensity and polarization.

\subsection{Separation of dust emission for intensity}

The CC coefficients at $v \geq 100 \mathrm{GHz}$ can be written as

$\left[\alpha_{v}^{\mathrm{I}}\right]_{353}^{3 \mathrm{~T}}=\alpha^{\mathrm{I}}\left(c_{353}^{3}\right)+\alpha_{v}^{\mathrm{I}}\left(d_{353}\right)$,

where $c_{353}^{3}$ and $d_{353}$ are the $353 \mathrm{GHz}$ correlated CMB and dust emission, respectively. The CMB CC coefficient is achromatic in $\mathrm{K}_{\mathrm{CMB}}$ units, i.e., in temperature units relative to the CMB blackbody spectrum. To remove the CMB contribution, we work with the differences of $\mathrm{CC}$ coefficients between two given frequencies. To measure the dust spectral index both in intensity and

\footnotetext{
4 http://archives.esac.esa.int
}

polarization, we choose to work with colour ratios defined between two given frequencies $v_{2}$ and $v_{1}$ as

$R_{v_{0}}^{\mathrm{I}}\left(v_{2}, v_{1}\right)=\frac{\left[\alpha_{v_{2}}^{\mathrm{I}}\right]_{353}^{3 \mathrm{~T}}-\left[\alpha_{v_{0}}^{\mathrm{I}}\right]_{353}^{3 \mathrm{~T}}}{\left[\alpha_{v_{1}}^{\mathrm{I}}\right]_{353}^{3 \mathrm{~T}}-\left[\alpha_{v_{0}}^{\mathrm{I}}\right]_{353}^{3 \mathrm{~T}}}=\frac{\alpha_{v_{2}}^{\mathrm{I}}\left(d_{353}\right)-\alpha_{v_{0}}^{\mathrm{I}}\left(d_{353}\right)}{\alpha_{v_{1}}^{\mathrm{I}}\left(d_{353}\right)-\alpha_{v_{0}}^{\mathrm{I}}\left(d_{353}\right)}$,

where $v_{0}$ represents the reference CMB frequency which is chosen to be $100 \mathrm{GHz}$ in the present analysis. To convert the measured colour ratio into $\beta_{\mathrm{d}}$ we follow earlier studies (Planck Collaboration Int. XVII 2014; Planck Collaboration XI 2014) by approximating the SED of the dust emission with a modified blackbody (MBB) spectrum (Planck Collaboration XXV 2011; Planck Collaboration XXIV 2011; Planck Collaboration XI 2014) given by

$\alpha_{v}^{\mathrm{I}}\left(d_{353}\right)=F_{v} C_{v} A_{\mathrm{d}} v^{\beta_{\mathrm{d}}} B_{v}\left(T_{\mathrm{d}}\right)$,

where $T_{\mathrm{d}}$ is the colour temperature and $\beta_{\mathrm{d}}$ is the spectral index of the dust emission. The factor $F_{v}$ takes into account the conversion from $\mathrm{MJy} \mathrm{sr}^{-1}$ (with the photometric convention $v I_{v}=$ constant) to $\mathrm{K}_{\mathrm{CMB}}$ units, while $C_{v}$ is the colour correction that depends on the value of $\beta_{\mathrm{d}}$ and $T_{\mathrm{d}}$. The colour correction is computed knowing the bandpass filters at the HFI frequencies (Planck Collaboration IX 2014) and the spectrum of the dust emission. Using Eq. (19), the colour ratio can be written as a function of $\beta_{\mathrm{d}}$ and $T_{\mathrm{d}}$ :

$$
\begin{aligned}
R_{v_{0}}^{\mathrm{I}}\left(v_{2}, v_{1}\right) & =\frac{F_{v_{2}} C_{v_{2}} v_{2}^{\beta_{\mathrm{d}}} B_{v_{2}}\left(T_{\mathrm{d}}\right)-F_{v_{0}} C_{v_{0}} v_{0}^{\beta_{\mathrm{d}}} B_{v_{0}}\left(T_{\mathrm{d}}\right)}{F_{v_{1}} C_{v_{1}} v_{1}^{\beta_{\mathrm{d}}} B_{v_{1}}\left(T_{\mathrm{d}}\right)-F_{v_{0}} C_{v_{0}} v_{0}^{\beta_{\mathrm{d}}} B_{v_{0}}\left(T_{\mathrm{d}}\right)} \\
& =g\left(\beta_{\mathrm{d}}, T_{\mathrm{d}}\right) .
\end{aligned}
$$

In Sect. 6.1, we use the three Planck maps, at 100, 217, and $353 \mathrm{GHz}$, to compute $R_{100}^{\mathrm{I}}(353,217)$ and measure the dust spectral index $\left(\beta_{\mathrm{d}, \mathrm{mm}}^{\mathrm{I}}\right)$ at microwave frequencies (or $\mathrm{mm}$ wavelengths), for each sky patch. In the next section, we explain how we determine $T_{\mathrm{d}}$.

\subsection{Measuring colour temperatures in intensity}

The dust temperatures inferred from an MBB fit of the Planck at $v \geq 353 \mathrm{GHz}$ and the IRAS $100 \mu \mathrm{m}$ sky maps at 5' resolution (Planck Collaboration XI 2014) cannot be used to compute mean temperatures within each sky patch because the fits are nonlinear. The two frequencies, Planck $857 \mathrm{GHz}$ and DIRBE $100 \mu \mathrm{m}(3000 \mathrm{GHz})$, which are close to the dust emission peak, are well suited to measure $T_{\mathrm{d}}$ for each sky patch. We use the Planck $353 \mathrm{GHz}$ map as a template to compute the colour ratio $R^{\mathrm{I}}(3000,857)$ over each sky patch, as described in Eq. (18). The superscript I on the colour ratio and $\beta_{\mathrm{d}}$ denote intensity. As the CMB signal is negligible at these frequencies, we work directly with the ratio $R^{\mathrm{I}}(3000,857)$, without subtracting the $100 \mathrm{GHz} \mathrm{CC}$ measure. We assume a mean dust spectral index at submm frequencies, $\beta_{\mathrm{d}, \mathrm{I} u b m m}$, of 1.50 . The choice of $\beta_{\mathrm{d}, \mathrm{submm}}^{\mathrm{I}}$ value is based on the MBB fit to the dust emissivities at $100 \mu \mathrm{m}$ and the Planck 353, 545 and $857 \mathrm{GHz}$ frequencies, for each sky patch. Due to the $\beta_{\mathrm{d} \text {,submm }}^{\mathrm{I}}-T_{\mathrm{d}}$ anti-correlation, the variations of the $\beta_{\mathrm{d} \text {,submm }}^{\mathrm{I}}$ values just increases the scatter of the $T_{\mathrm{d}}$ values by about $20 \%$ as compared to $T_{\mathrm{d}}$ values derived using fixed $\beta_{\mathrm{d}, \text { submm }}^{\mathrm{I}}$. However, the $T_{\mathrm{d}}$ values from the MBB fits are closely correlated with $T_{\mathrm{d}}$ values determined using the ratio $R^{\mathrm{I}}(3000,857)$ and a fixed spectral index. We use the colour ratio $R^{\mathrm{I}}(3000,857)$ and 


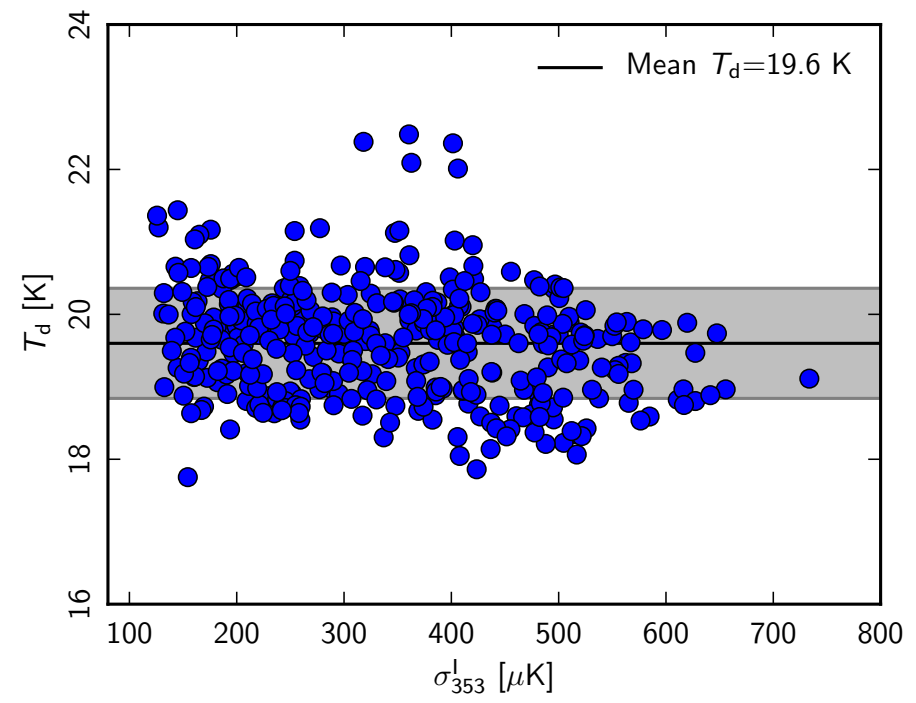

Fig. 2. Dust colour temperatures, $T_{\mathrm{d}}$, computed from $R^{\mathrm{I}}(3000,857)$, are plotted versus the local dispersion of the $353 \mathrm{GHz}$ intensity template, $\sigma_{353}^{\mathrm{I}}$. The mean $T_{\mathrm{d}}$ is $19.6 \mathrm{~K}$, with the $1 \sigma$ dispersion of $0.8 \mathrm{~K}$ across sky patches (shaded area).

mean $\beta_{\mathrm{d} \text {,submm }}^{\mathrm{I}}=1.50$ to estimate $T_{\mathrm{d}}$ values for each sky patch by inverting the relation given in Eq. (20).

In Fig. 2, we plot the derived $T_{\mathrm{d}}$ versus the local brightness dispersion of the Planck $353 \mathrm{GHz}$ template in intensity $\left(\sigma_{353}^{\mathrm{I}}\right)$. We point out that $\sigma_{353}^{\mathrm{I}}$ is not an uncertainty in the $353 \mathrm{GHz}$ intensity template. The mean value of $T_{\mathrm{d}}$ over our mask at intermediate Galactic latitudes is $19.6 \mathrm{~K}$. The $1 \sigma$ dispersion of $T_{\mathrm{d}}$ over the $400 \mathrm{sky}$ patches is $0.8 \mathrm{~K}$. This value is slightly smaller that the mean value at high Galactic latitudes, $20.4 \pm 1.1 \mathrm{~K}$ for $\beta_{\mathrm{d} \text {,submm }}^{\mathrm{I}}=1.57 \pm 0.11^{5}$, we obtained repeating the dust-HI correlation analysis of Planck Collaboration Int. XVII (2014) on the same full-mission Planck data.

The choice of $\beta_{\mathrm{d} \text {,submm }}^{\mathrm{I}}$ used in this paper is different from the one derived from the analysis of high Galactic latitude data (Planck Collaboration Int. XVII 2014) and the analysis of the whole sky (Planck Collaboration XI 2014) using public release Planck 2013 data. This difference results from a change in the photometric calibration by $1.9 \%,-2.2 \%,-3.5 \%$, at 353,545 and $857 \mathrm{GHz}$, between the DX11d and the Planck 2013 data. The new calibration factors make the mean $\beta_{\mathrm{d} \text {,submm }}^{\mathrm{I}}$ slightly smaller and $T_{\mathrm{d}}$ slightly higher. To estimate uncertainties on $\beta_{\mathrm{d}, \text { submm }}^{\mathrm{I}}$, we run a set of Monte-Carlo simulations that take into account the absolute and relative calibration uncertainties present in the DIRBE and the Planck full-mission HFI data at $v \geq 353 \mathrm{GHz}$. We assume that the MBB spectrum is a good fit to the data and apply $1 \sigma$ photometric uncertainties of $1 \%, 7 \%, 7 \%$, and $13 \%$ at $353,545,857$, and $3000 \mathrm{GHz}$ respectively. To get multiple SED realizations, we vary the MBB spectrum within the photometric uncertainty at each frequency used for the fit, independently of others. Then we perform the MBB SED fit and find that the $1 \sigma$ dispersion on the mean value of $\beta_{\text {d,submm }}^{\mathrm{I}} 0.16$ (syst.). The new value of $\beta_{\mathrm{d}, \mathrm{submm}}^{\mathrm{I}}=1.50$ is well within the range of values and systematic uncertainties quoted in Table 3 of Planck Collaboration XI (2014, using 2013 Planck data) for the same region of the sky.

\footnotetext{
These values are derived from a grey-body fit of dust SED at $v \geq$ $353 \mathrm{GHz}$. The error-bar on the dust temperature is associated with that on the spectral index.
}

\subsection{Separation of $C M B$ emission in intensity}

The CC coefficient, derived in Eq. (8), contains the CMB contribution that is achromatic in $\mathrm{K}_{\mathrm{CMB}}$ units. We determine this CMB contribution assuming that the dust emission is well approximated by a MBB spectrum from 100 to $353 \mathrm{GHz}$. For each sky patch, we use the values of $\beta_{\mathrm{d}, \mathrm{mm}}^{\mathrm{I}}$ and $T_{\mathrm{d}}$ from Sects. 6.1 and 5.2. We solve for two parameters, the CMB contribution, $\alpha^{\mathrm{I}}\left(c_{353}^{3}\right)$, and the dust amplitude, $A_{\mathrm{d}}^{\mathrm{I}}$, by minimizing

$\chi_{\mathrm{s}}^{2}=\sum_{v}\left(\frac{\left[\alpha_{v}^{\mathrm{I}}\right]_{353}^{3 \mathrm{~T}}-\alpha^{\mathrm{I}}\left(c_{353}^{3}\right)-F_{v} C_{v} A_{\mathrm{d}}^{\mathrm{I}} v^{\beta_{\mathrm{d}, \mathrm{mm}}^{\mathrm{I}}} B_{v}\left(T_{\mathrm{d}}\right)}{\sigma_{\alpha_{v}^{\mathrm{I}}}}\right)^{2}$,

where $\sigma_{\alpha_{v}^{\mathrm{I}}}$ is the uncertainty on the CC coefficient, determined using the Monte Carlo simulations (Appendix B). The joint spectral fit of $\alpha^{\mathrm{I}}\left(c_{353}^{3}\right), \beta_{\mathrm{d}, \mathrm{mm}}^{\mathrm{I}}, T_{\mathrm{d}}$, and $A_{\mathrm{d}}^{\mathrm{I}}$ leads to a degeneracy between the fitted parameters. To avoid this problem, we fix the values of $\beta_{\mathrm{d}, \mathrm{mm}}^{\mathrm{I}}$ and $T_{\mathrm{d}}$ for each sky patch based on the colour ratios, independent of the value of $\alpha^{\mathrm{I}}\left(c_{353}^{3}\right)$. The CMB contributions are subtracted from the $\mathrm{CC}$ coefficients at all frequencies, including the LFI and WMAP data not used in the fit. After CMB subtraction, the CC coefficient $\left(\tilde{\alpha}_{v}^{\mathrm{I}}\right)$ for the $353 \mathrm{GHz}$ template is

$\left[\tilde{\alpha}_{v}^{\mathrm{I}}\right]_{353}^{3 \mathrm{~T}}=\left[\alpha_{v}^{\mathrm{I}}\right]_{353}^{3 \mathrm{~T}}-\alpha^{\mathrm{I}}\left(c_{353}^{3}\right)=\alpha_{v}^{\mathrm{I}}\left(d_{353}\right)$.

We perform the same exercise on the one- and two-template fits to derive the $\mathrm{CMB}$ subtracted $\mathrm{CC}$ coefficients.

\subsection{Separation of dust emission for polarization}

As for our analysis of the intensity data, we write the $353 \mathrm{GHz}$ correlated polarized CC coefficients at $v \geq 100 \mathrm{GHz}$ as

$\left[\alpha_{v}^{\mathrm{P}}\right]_{353}^{1 \mathrm{~T}}=\alpha^{\mathrm{P}}\left(c_{353}^{1}\right)+\alpha_{v}^{\mathrm{P}}\left(d_{353}\right)$,

where $c_{353}^{1}$ and $d_{353}$ are the CMB and dust polarized emission correlated with the 353 polarization templates. The contributions from synchrotron and AME to the polarized $\mathrm{CC}$ coefficients are assumed to be negligible at HFI frequencies. Like for intensity in Eq. (20), we compute $R_{100}^{\mathrm{P}}(353,217)$ combining the three polarized CC coefficients at 100, 217 and $353 \mathrm{GHz}$. We assume that the temperature of the dust grains contributing to the polarization is the same as that determined for the dust emission in intensity (Sect. 5.2), and derive $\beta_{\mathrm{d}, \mathrm{mm}}^{\mathrm{P}}$ at microwave frequencies.

To separate the contribution of dust and the CMB to the polarized CC coefficients, we follow the method described in Sect. 5.3, and rely on the Monte Carlo simulations described in Appendix B to estimate uncertainties. The CMB contribution is subtracted at all frequencies, including the LFI and WMAP data.

\section{Dust spectral index for intensity}

Here we estimate the dust spectral index $\beta_{\mathrm{d}, \mathrm{mm}}^{\mathrm{I}}$ at microwave frequencies $(v \leq 353 \mathrm{GHz})$ and $\mathrm{mm}$ wavelengths. We present the results of the data analysis and estimate the uncertainties, including possible systematic effects.

\subsection{Measuring $\beta_{d, m m}^{l}$}

We use the three full mission Planck maps, at 100, 217, and $353 \mathrm{GHz}$, to derive a mean $\beta_{\mathrm{d}, \mathrm{mm}}^{\mathrm{I}}$ using the three-template fit, assuming an MBB spectrum for the dust emission (Sect. 5.1). 

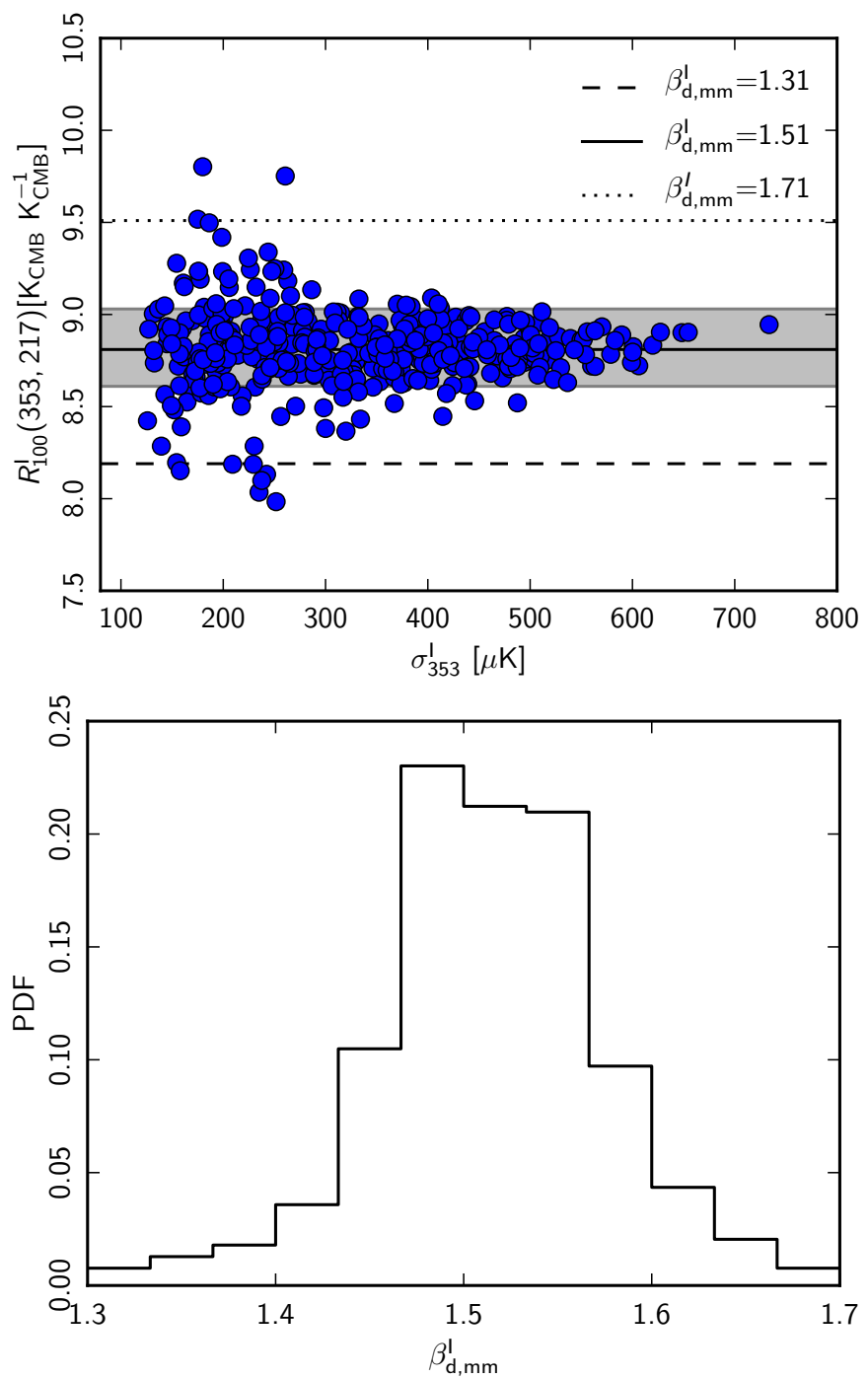

Fig. 3. Top: colour ratio $R_{100}^{\mathrm{I}}(353,217)$ versus dispersion of the $353 \mathrm{GHz}$ template $\sigma_{353}^{\mathrm{I}}$ for each sky patch. The $1 \sigma$ dispersion of the $R_{100}^{\mathrm{I}}(353,217)$ values is shown as the shaded grey area. The mean ratio corresponds to a spectral index of 1.51 (solid line) for a mean $T_{\mathrm{d}}=19.6 \mathrm{~K}$. Bottom: probability distribution function (PDF) of the $\beta_{\mathrm{d}, \mathrm{mm}}^{\mathrm{I}}$ values derived from $R_{100}^{\mathrm{I}}(353,217)$ using the specific value of $T_{\mathrm{d}}$ for each sky patch. The measured $1 \sigma$ dispersion of $\beta_{\mathrm{d}, \mathrm{mm}}^{\mathrm{I}}$ is 0.07 .

The 217 and $353 \mathrm{GHz}$ maps have the highest signal-to-noise ratio for dust emission at microwave frequencies, whereas the $100 \mathrm{GHz}$ map is used as a reference frequency to subtract the CMB contribution at the CC level. We estimate $R_{100}^{\mathrm{I}}(353,217)$ for each sky patch using the relation given by Eq. (18). The values of $R_{100}^{\mathrm{I}}(353,217)$ are plotted in top panel of Fig. 3 as a function of $\sigma_{353}^{\mathrm{I}}$, which allows us to identify the statistical noise and systematic effects due to uncertainties on the CC coefficients. Our Monte Carlo simulations (Appendix B) show that the uncertainties on $R_{100}^{\mathrm{I}}(353,217)$ scale approximately as the inverse square-root of $\sigma_{353}^{\mathrm{I}}$, and that the scatter in the measured $R_{100}^{\mathrm{I}}(353,217)$ for sky patches with low $\sigma_{353}^{\mathrm{I}}$ is due to data noise.

For each sky patch, we derive $\beta_{\mathrm{d}, \mathrm{mm}}^{\mathrm{I}}$ from $R_{100}^{\mathrm{I}}(353,217)$ by inverting Eq. (20) for the values of $T_{\mathrm{d}}$ derived in Sect. 5.2. The histogram of $\beta_{\mathrm{d}, \mathrm{mm}}^{\mathrm{I}}$ for all sky patches is presented in the bottom panel of Fig. 3. The mean value of $\beta_{\mathrm{d}, \mathrm{mm}}^{\mathrm{I}}$ from the $400 \mathrm{sky}$ patches is 1.514 (round-off to 1.51 ) with $1 \sigma$ dispersion of 0.065

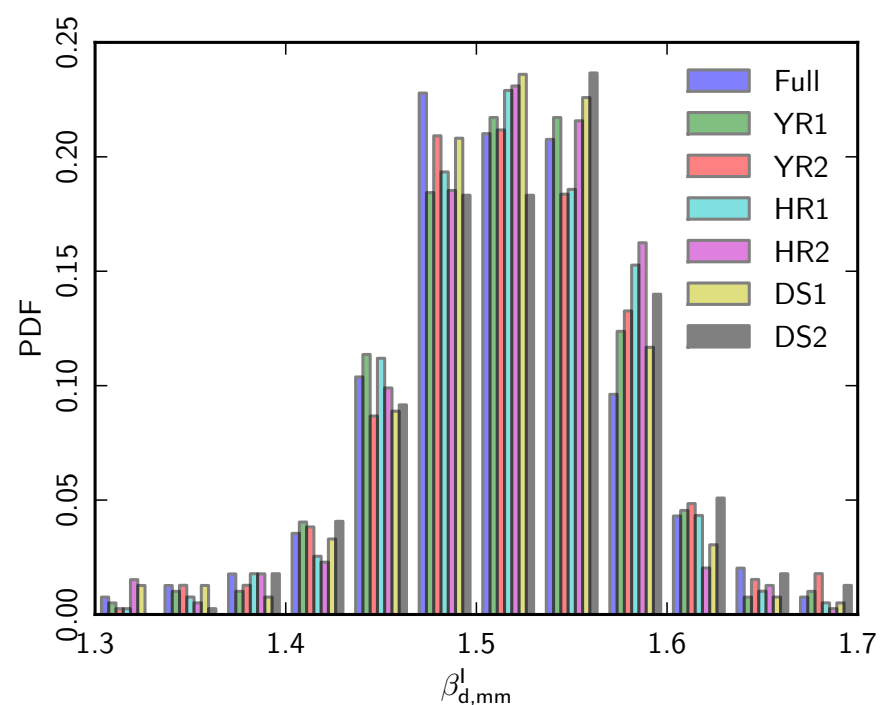

Fig. 4. Same plot as in the bottom panel of Fig. 3, including our results for the subsets of the Planck data listed in Table 2. The bin per bin measurements of $\beta_{\mathrm{d}, \mathrm{mm}}^{\mathrm{I}}$ using subsets of the Planck data are compatible with the one obtained using the full mission data (Sect. 6.1).

Table 2. Dust spectral indices for intensity derived applying the threetemplate fit on distinct subsets of the Planck data (Sect. 2.1.2).

\begin{tabular}{lll}
\hline \hline Data sets $\ldots \ldots \ldots \ldots \ldots$ & $\beta_{\mathrm{d}, \mathrm{mm}}^{\mathrm{I}}$ \\
\hline Full $\ldots \ldots \ldots \ldots \ldots \ldots$ & 1.514 \\
YR1 $\ldots \ldots \ldots \ldots \ldots \ldots$ & 1.514 \\
YR2 $\ldots \ldots \ldots \ldots \ldots \ldots$ & 1.519 \\
HR1 $\ldots \ldots \ldots \ldots \ldots \ldots$ & 1.515 \\
HR2 $\ldots \ldots \ldots \ldots \ldots \ldots \ldots$ & 1.518 \\
DS1 $\ldots \ldots \ldots \ldots \ldots \ldots \ldots$ & 1.514 \\
DS2 $\ldots \ldots \ldots \ldots \ldots \ldots \ldots$ & 1.520 \\
\hline
\end{tabular}

Notes. Here the index "Full" refers to the full mission Planck 2014 data, which is used in Sect. 6.1 to produce Fig. 3. The scatter of the seven measurements for the subsets of the Planck data is within the $1 \sigma$ statistical uncertainty on the mean $\beta_{\mathrm{d}, \mathrm{mm}}^{\mathrm{I}}$.

(round-off to 0.07). The statistical uncertainty on the mean $\beta_{\mathrm{d}, \mathrm{Im}}^{\mathrm{I}}$ is 0.01 , which is computed from the $1 \sigma$ deviation divided by the square root of the number of independent sky patches $\left(400 / N_{\text {visit }}\right)$ used. This estimate of the statistical error bar on $\beta_{\mathrm{d}, \mathrm{mm}}^{\mathrm{I}}$ takes into account the uncertainties associated with the chance correlation between the dust template and emission components (CO lines, point sources, the CIB anisotropies and the ZLE) not fitted with templates. It also includes uncertainties on the subtraction of the CMB contribution.

\subsection{Uncertainties on $\beta_{d, m m}^{l}$}

We use the full mission Planck intensity maps as a reference data for the mean dust spectral index value. To assess the systematic uncertainties on the mean spectral index, we repeat our CC analysis on maps made with subsets of the Planck data (Sect. 2.1.2), keeping the same ZLE-subtracted Planck $353 \mathrm{GHz}$ map as a template. For each set of maps, we compute the mean $\beta_{\mathrm{d}, \mathrm{mm}}^{\mathrm{I}}$ from $R_{100}^{\mathrm{I}}(353,217)$ values. Table 2 lists the $\beta_{\mathrm{d}, \mathrm{mm}}^{\mathrm{I}}$ values derived from the three-template fit applied to each data sub-set. The six measurements of $\beta_{\mathrm{d}, \mathrm{mm}}^{\mathrm{I}}$ from various data splits are within the $1 \sigma$ 


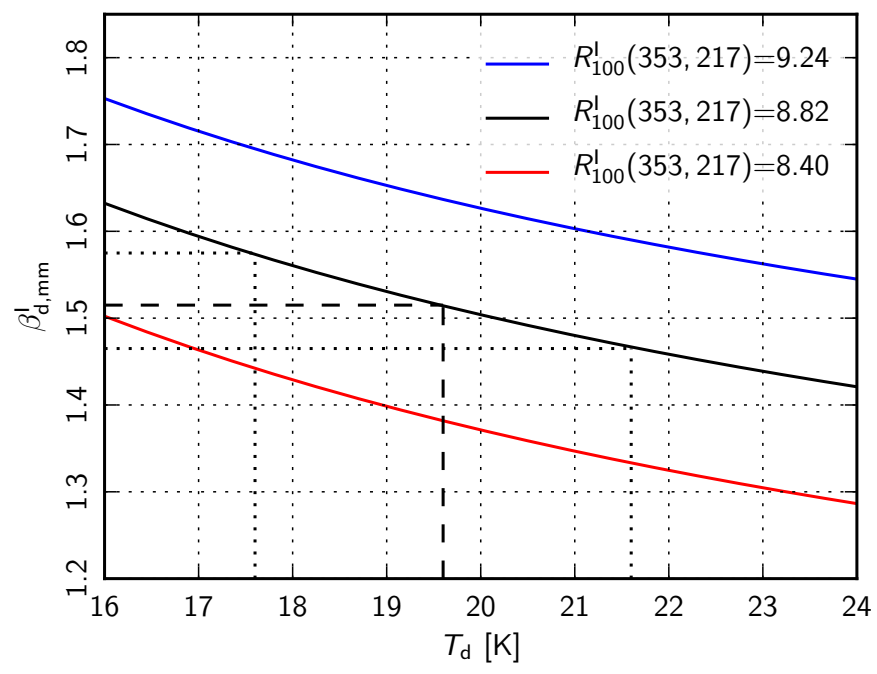

Fig. 5. Variation of $\beta_{\mathrm{d}, \mathrm{mm}}^{\mathrm{I}}$ with $T_{\mathrm{d}}$ for constant values of $R_{100}^{\mathrm{I}}(353,217)$. The dashed line corresponds to a mean $T_{\mathrm{d}}=19.6 \mathrm{~K}$ and $\beta_{\mathrm{d}, \mathrm{mm}}^{\mathrm{I}}=1.51$ for the best-fit value of $R_{100}^{\mathrm{I}}(353,217)=8.82$. The two dotted line lines correspond to a change in $T_{\mathrm{d}}$ of $\pm 2 \mathrm{~K}$ about its mean value, resulting in change of $\beta_{\mathrm{d}, \mathrm{mm}}^{\mathrm{I}}$ value of \pm 0.05 .

statistical uncertainties on the mean intensity dust spectral index. We find a mean dust spectral index $\beta_{\mathrm{d}, \mathrm{mm}}^{\mathrm{I}}=1.51 \pm 0.01$ (stat.). This spectral index is very close to the mean index of 1.50 at sub-mm wavelengths we derived from MBB fits to the Planck data at $v \geq 353 \mathrm{GHz}$ in Sect. 5.2.

\subsection{Dependence of $\beta_{d, m m}^{l}$ on the choice of $\beta_{d, \text { submm }}^{l}$}

In Fig. 5 we plot $R_{100}^{\mathrm{I}}(353,217)$ as a function of $\beta_{\mathrm{d}, \mathrm{mm}}^{\mathrm{I}}$ and $T_{\mathrm{d}}$. The central black line corresponds to the median value of $R_{100}^{\mathrm{I}}(353,217)$ obtained using the $\mathrm{CC}$ analysis. Figure 5 shows that varying $T_{\mathrm{d}}$ by $\pm 2 \mathrm{~K}, \mathrm{a} \pm 2.5 \sigma$ deviation from the mean value, changes $\beta_{\mathrm{d}, \mathrm{mm}}^{\mathrm{I}}$ by \pm 0.05 . To estimate $\beta_{\mathrm{d}, \mathrm{mm}}^{\mathrm{I}}$, we use $T_{\mathrm{d}}$, which in turn depends on $\beta_{\mathrm{d} \text {,submm }}^{\mathrm{I}}$. We repeat our analysis with two different starting values of $\beta_{\mathrm{d} \text {,submm }}^{\mathrm{I}}$, which are within $1 \sigma$ systematics uncertainties derived in Sect. 5.2. For the values of $\beta_{\mathrm{d}, \mathrm{submm}}^{\mathrm{I}}=1.34$ and 1.66 , we find $\beta_{\mathrm{d}, \mathrm{mm}}^{\mathrm{I}}=1.50$ and 1.53 , respectively. This is due to the fact that in Rayleigh-Jeans limit, the effect of $T_{\mathrm{d}}$ is low, and the shape of the spectrum is dominated by $\beta_{\mathrm{d}, \mathrm{mm}}^{\mathrm{I}}$. Our determination of mean $\beta_{\mathrm{d}, \mathrm{mm}}^{\mathrm{I}}$ is robust and independent of the initial choice of $\beta_{\mathrm{d} \text {,submm }}^{\mathrm{I}}$ used for the analysis.

\subsection{Alternative approach of measuring $\beta_{d, m m}^{l}$}

To derive the dust spectral index from $R_{100}^{\mathrm{I}}(353,217)$, we assume an MBB spectrum for the dust emission between 100 and $353 \mathrm{GHz}$ (Sect. 5.1). To validate this assumption, we repeat our $\mathrm{CC}$ analysis with Planck maps corrected for $\mathrm{CMB}$ anisotropies using the CMB map from the spectral matching independent component analysis (SMICA, Planck Collaboration XII 2014). We infer $\beta_{\mathrm{d}, \mathrm{mm}}^{\mathrm{I}}$ (SMICA) directly from the ratio between the 353 and $217 \mathrm{GHz}$ CC coefficients without subtracting the $100 \mathrm{GHz} \mathrm{CC}$ coefficient, i.e.,

$R_{\text {SMICA }}^{\mathrm{I}}(353,217)=\frac{\left[\alpha_{353}^{\prime}\right]_{(353-\mathrm{SMICA})}^{1 \mathrm{~T}}}{\left[\alpha_{217}^{\prime}\right]_{(353-\mathrm{SMICA})}^{1 \mathrm{~T}}}$,

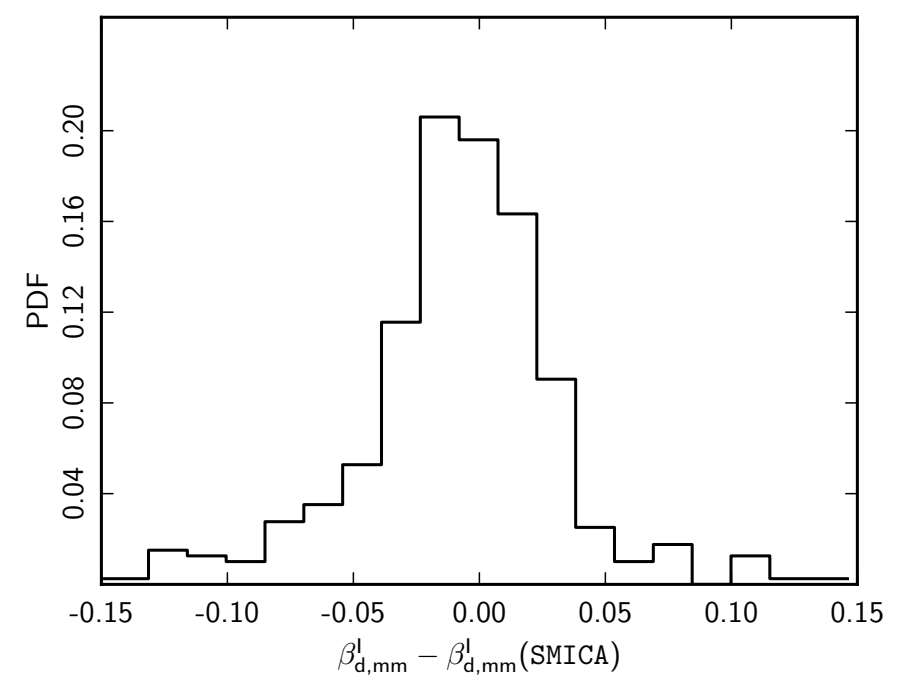

Fig. 6. Histogram of the difference between the spectral indices $\beta_{\mathrm{d}, \mathrm{mm}}^{\mathrm{I}}$ from Eq. (20) and $\beta_{\mathrm{d}, \mathrm{Im}}^{\mathrm{I}}$ (SMICA) from Eq. (24), where we applied the $\mathrm{CC}$ analysis to Planck maps corrected for $\mathrm{CMB}$ anisotropies with the SMICA CMB map.

where $\alpha^{\prime}$ refers to the CC coefficients computed with maps corrected for CMB anisotropies using the SMICA map. The histogram of the difference between the two sets of spectral indices $\beta_{\mathrm{d}, \mathrm{mm}}^{\mathrm{I}}$ and $\beta_{\mathrm{d}, \mathrm{mm}}^{\mathrm{I}}$ (SMICA) is presented in Fig. 6. The mean difference between the two estimates is zero.

\subsection{Comparison with other studies}

Our determination of the spectral index $\beta_{\mathrm{d}, \mathrm{mm}}^{\mathrm{I}}$ of the dust emission in intensity at intermediate Galactic latitudes may be compared with the results from similar analyses of the Planck data. In Planck Collaboration Int. XVII (2014), the CC analysis has been applied to the Planck data at high Galactic latitudes $(b<$ $-30^{\circ}$ ) using an HI map as a dust template free from CIB and CMB anisotropies. This is a suitable template to derive the spectral dependence of dust emission at high Galactic latitudes. The same methodology of colour ratios has been used in that work. The mean dust spectral index, $\beta_{\mathrm{d}, \mathrm{mm}}^{\mathrm{I}}=1.53 \pm 0.03$, from Planck Collaboration Int. XVII (2014) agrees with the mean value we find in this paper. In an analysis of the diffuse emission in the Galactic plane, the spectral index of dust at millimetre wavelengths is found to increase from $\beta_{\mathrm{d}, \mathrm{mm}}^{\mathrm{I}}=1.54$, for lines of sight where the medium is mostly atomic, to $\beta_{\mathrm{d}, \mathrm{mm}}^{\mathrm{I}}=1.66$, where the medium is predominantly molecular (Planck Collaboration Int. XIV 2014). The three studies indicate that the spectral in$\operatorname{dex} \beta_{\mathrm{d}, \mathrm{mm}}^{\mathrm{I}}$ is remarkably similar over the diffuse ISM observed at high and intermediate Galactic latitudes, and in the Galactic plane.

\section{Spectral energy distribution of dust intensity}

In this section, we derive the mean SED of dust emission for intensity with its uncertainties from our CC analysis. The detailed spectral modelling of the dust SED is discussed in Sect. 7.2.

\subsection{Mean dust SED}

We use the full mission Planck maps for the spectral modelling of the dust SED in intensity. The mean SED is obtained by averaging the $\mathrm{CC}$ coefficients after $\mathrm{CMB}$ subtraction 
Planck Collaboration: Frequency dependence of thermal emission from Galactic dust in intensity and polarization
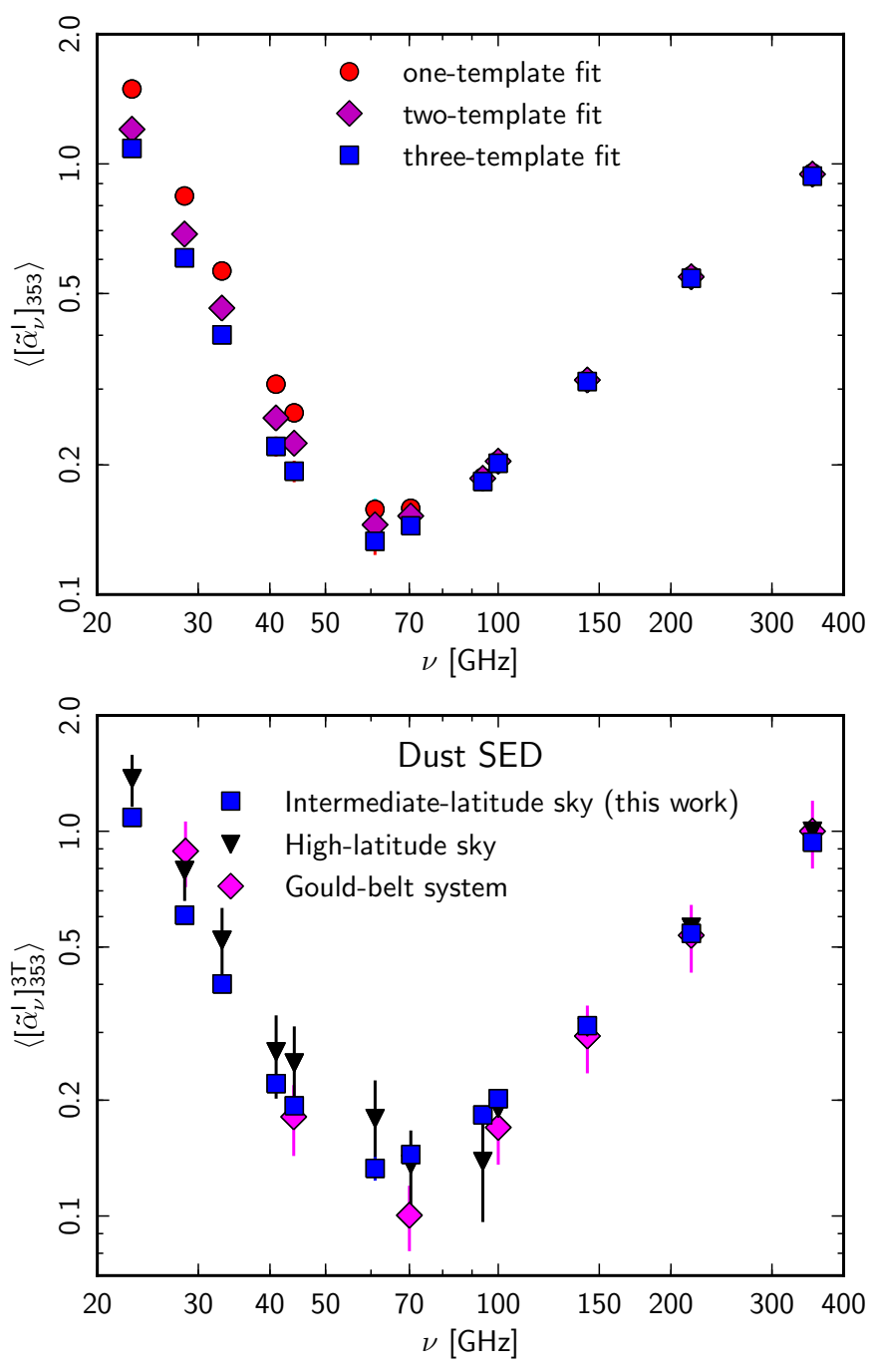

Fig. 7. Top: the three $\mathrm{SEDs}$ in $\mathrm{K}_{\mathrm{RJ}}$ units, normalized to 1 at $353 \mathrm{GHz}$, obtained by averaging, $\mathrm{CMB}$ corrected, $\mathrm{CC}$ coefficients from the fits with one (cyan circles), two (blue circles) and three templates (red circles). The uncertainties at each frequency are estimated using the subsets of Planck and WMAP data. Bottom: our SED from the three-template fit (red circles) is compared with the dust SED in Planck Collaboration Int. XII (2013) for the Gould Belt system (squares) and that at high latitude sky (inverted triangles) obtained applying the dust-HI correlation analysis in Planck Collaboration Int. XVII (2014) to the full Planck mission data.

(see Sect. 5.3) over all sky patches. The mean SED is expressed in $\mathrm{K}_{\mathrm{RJ}}$ units, normalized to 1 at $353 \mathrm{GHz}$. The three SEDs obtained from the fits with one, two, and three templates are shown in Fig. 7. The SED values obtained with the three templates fit are listed in Table 3. The three SEDs are identical at the highest frequencies. They differ at $v<100 \mathrm{GHz}$ due to the non-zero correlation between the $353 \mathrm{GHz}$ dust template with synchrotron and free-free emission. At $23 \mathrm{GHz}$, after $\mathrm{CMB}$ correction the CC coefficient from the fit with three templates is lower by 9 and $35 \%$ from those derived from the fits with two and one template, respectively. The $9 \%$ difference accounts for the freefree emission correlated with dust and the $35 \%$ difference for the combination of both synchrotron and free-free emission correlated with dust. At these low frequencies, the fit with three templates provides the best separation of the dust from the synchrotron and free-free emission. It is this SED that we call the dust SED hereafter.
Our dust SED is similar to that measured for the Gould Belt system (Planck Collaboration Int. XII 2013) and at high Galactic latitudes (Planck Collaboration Int. XVII 2014). It shows the thermal dust emission at $v \geq 70 \mathrm{GHz}$ and is dominated by AME at lower frequencies. At WMAP frequencies, our dust SED is similar to that obtained using the FDS $94 \mathrm{GHz}$ map as a dust template in Davies et al. (2006) and Ghosh et al. (2012). The mean dust SED derived using the three-template fit depends on the correction of the DDD $\mathrm{H} \alpha$ map for dust scattering and extinction (Dickinson et al. 2003; Witt et al. 2010; Brandt \& Draine 2012; Bennett et al. 2013). In Appendix C, we study the impact of both assumptions on the mean dust SED, which are within a few percent at frequencies below $70 \mathrm{GHz}$ and have no impact on frequencies above $100 \mathrm{GHz}$.

The total uncertainty on the dust SED includes the intercalibration uncertainties on the data, the statistical uncertainties estimated from the variations of the $\mathrm{CC}$ coefficients across the sky patches, and the uncertainties due to the CMB subtraction, as discussed for $\beta_{\mathrm{d}, \mathrm{mm}}^{\mathrm{I}}$ in Sect. 6.1. The inter-calibration uncertainties $\left(c_{v}\right)$ for Planck and WMAP data are given in Table 3. The statistical uncertainties are computed from the $1 \sigma$ dispersion of the CC coefficients over the 400 sky patches divided by the square root of the number of independent sky patches $\left(400 / N_{\text {visit }}\right)$. All three types of uncertainty, listed in Table 3, are added together in quadrature to compute the total uncertainty on the mean dust SED. They are shown in Fig. 7, but most do not appear because they are smaller than the size of the symbols.

\subsection{Parametric modelling for intensity dust SED}

A spectral fit of the dust SED is required to separate the thermal dust emission from the AME. We continue to use an MBB spectrum for the thermal dust emission, and consider two models with different spectra for the AME.

- Model DI+AI: in this first approach, we use the analytical model of the AME (AI) introduced by Bonaldi et al. (2007), which is a parabola in the $\log I_{v}-\log v$ plane, parameterized by the peak frequency $\left(v_{\mathrm{p}}\right)$ and the slope $-m_{60}$ at $60 \mathrm{GHz}$. The AME model $\left(M_{\mathrm{a}}\right)$ normalized at $23 \mathrm{GHz}$ (in $\mathrm{K}_{\mathrm{RJ}}$ units) is given by:

$$
\begin{aligned}
& \log M_{\mathrm{a}}=-\left(\frac{m_{60} \log v_{\mathrm{p}}}{\log \left(v_{\mathrm{p}} / 60 \mathrm{GHz}\right)}+2\right) \log \left(\frac{v}{23 \mathrm{GHz}}\right) \\
& +\frac{m_{60}}{2 \log \left(v_{\mathrm{p}} / 60 \mathrm{GHz}\right)}\left[(\log (v / 1 \mathrm{GHz}))^{2}-(\log 23)^{2}\right] .
\end{aligned}
$$

This model is a good fit to spectra of dipole emission from small spinning dust particles computed with the SPDUST code (Ali-Haïmoud et al. 2009; Silsbee et al. 2011). The second component is the MBB spectrum of the thermal dust emission (DI) with free parameter $\beta_{\mathrm{d}, \mathrm{mm}}^{\mathrm{I}}$. The total model is written in $\mathrm{K}_{\mathrm{RJ}}$ units normalized to 1 at the frequency $v_{\text {ref }}=353 \mathrm{GHz}$ :

$\left\langle\left[\tilde{\alpha}_{v}^{\mathrm{I}}\right]_{353}^{3 \mathrm{~T}}\right\rangle=A_{\mathrm{a}}^{\mathrm{I}} M_{\mathrm{a}}+\left(\frac{v}{v_{\text {ref }}}\right)^{\beta_{\mathrm{d}, \mathrm{mm}}^{\mathrm{I}}-2} \frac{B_{v}\left(T_{\mathrm{d}}\right)}{B_{v_{\text {ref }}}\left(T_{\mathrm{d}}\right)}$,

where $A_{\mathrm{a}}^{\mathrm{I}}$ is the amplitude of AME and $\beta_{\mathrm{d}, \mathrm{mm}}^{\mathrm{I}}$ is the spectral index of thermal dust emission at microwave frequencies. We fix $T_{\mathrm{d}}=19.6 \mathrm{~K}$ from Sect. 5.2. The four free parameters of the model are $A_{\mathrm{a}}^{\mathrm{I}}, m_{60}, \nu_{\mathrm{p}}$ and $\beta_{\mathrm{d}, \mathrm{mm}}^{\mathrm{I}}$.

- Model DI+AII: in this second approach, the AME component (AII) is a linear combination of two spinning dust components arising from the typical cold neutral medium (CNM) 
Table 3. Mean microwave SEDs obtained from the fit with one, two, and three templates using the CC analysis.

\begin{tabular}{|c|c|c|c|c|c|c|c|c|c|c|c|c|}
\hline \multirow[b]{2}{*}{ Quantity } & \multicolumn{12}{|c|}{$\begin{array}{c}\text { Experiment } \\
\text { Frequency }[\mathrm{GHz}]\end{array}$} \\
\hline & $\begin{array}{l}\text { WMAP } \\
23\end{array}$ & $\begin{array}{l}\text { Planck } \\
28.4\end{array}$ & $\begin{array}{l}\text { WMAP } \\
33\end{array}$ & $\begin{array}{l}\text { WMAP } \\
41\end{array}$ & $\begin{array}{c}\text { Planck } \\
44.1\end{array}$ & $\begin{array}{l}\text { WMAP } \\
61\end{array}$ & $\begin{array}{c}\text { Planck } \\
70.4\end{array}$ & $\begin{array}{l}\text { WMAP } \\
94\end{array}$ & $\begin{array}{l}\text { Planck } \\
100\end{array}$ & $\begin{array}{l}\text { Planck } \\
143\end{array}$ & $\begin{array}{l}\text { Planck } \\
217\end{array}$ & $\begin{array}{c}\text { Planck } \\
353\end{array}$ \\
\hline$\left\langle\left[\tilde{\alpha}_{v}^{\mathrm{I}}\right]_{353}^{3 \mathrm{~T}}\right\rangle \ldots$ & 1.1202 & 0.5813 & 0.3955 & 0.2223 & 0.1857 & 0.1335 & 0.1361 & 0.1745 & 0.2108 & 0.3058 & 0.5837 & 1.0000 \\
\hline$\sigma_{\text {stat }} \ldots \ldots \ldots$ & 0.0319 & 0.0175 & 0.0126 & 0.0073 & 0.0056 & 0.0038 & 0.0023 & 0.0038 & 0.0023 & 0.0029 & 0.0045 & 0.0073 \\
\hline$c_{\nu}[\%]$. & 1.0 & 1.0 & 1.0 & 1.0 & 1.0 & 1.0 & 0.5 & 1.0 & 0.5 & 0.5 & 0.5 & 1.0 \\
\hline$\sigma_{\mathrm{cmb}}$ & 0.0086 & 0.0083 & 0.0085 & 0.0083 & 0.0081 & 0.0079 & 0.0037 & 0.0070 & 0.0035 & 0.0026 & 0.0015 & 0.0007 \\
\hline$\sigma_{\text {tot }}$ & 0.0346 & 0.0201 & 0.0156 & 0.0113 & 0.0100 & 0.0089 & 0.0044 & 0.0081 & 0.0043 & 0.0041 & 0.0055 & 0.0119 \\
\hline$\sigma_{\text {tot }}$ & 32.3 & 29.0 & 25.3 & 19.7 & 18.6 & 15.1 & 31.0 & 21.6 & 49.3 & 74.3 & 106.9 & 83.9 \\
\hline$C$. & 1.0732 & 1.0000 & 1.0270 & 1.0480 & 1.0000 & 1.0450 & 0.9810 & 0.9927 & 1.0877 & 1.0191 & 1.1203 & 1.1114 \\
\hline$U$ & 0.9864 & 0.9487 & 0.9723 & 0.9577 & 0.9328 & 0.9091 & 0.8484 & 0.7998 & 0.7942 & 0.5921 & 0.3343 & 0.0751 \\
\hline$\left\langle\left[\tilde{\alpha}_{v}^{\mathrm{I}}\right]_{353}^{1 \mathrm{~T}}\right\rangle \ldots$. & 1.5120 & 0.7952 & 0.5469 & 0.3046 & 0.2494 & 0.1556 & 0.1469 & 0.1755 & 0.2100 & 0.3037 & 0.5817 & 1.0000 \\
\hline$\sigma_{\text {tot }}^{1 \mathrm{~T}} \ldots \ldots \ldots$ & 0.0565 & 0.0304 & 0.0219 & 0.0139 & 0.0118 & 0.0089 & 0.0053 & 0.0077 & 0.0047 & 0.0042 & 0.0050 & 0.0113 \\
\hline$\left\langle\left[\tilde{\alpha}_{v}^{\mathrm{I}}\right]_{353}^{2 \mathrm{~T}}\right\rangle \ldots$ & 1.2274 & 0.6530 & 0.4515 & 0.2561 & 0.2135 & 0.1443 & 0.1419 & 0.1756 & 0.2108 & 0.3050 & 0.5823 & 1.0000 \\
\hline$\sigma_{\text {tot }}^{2 \mathrm{~T}} \ldots \ldots \ldots$ & 0.0369 & 0.0218 & 0.0168 & 0.0116 & 0.0102 & 0.0084 & 0.0045 & 0.0075 & 0.0042 & 0.0039 & 0.0051 & 0.0115 \\
\hline
\end{tabular}

Notes. $\left\langle\left[\tilde{\alpha}_{v}^{I}\right]_{353}^{3 \mathrm{~T}}\right\rangle \equiv$ mean dust SED in $\mathrm{K}_{\mathrm{RJ}}$ units, normalized to 1 at $353 \mathrm{GHz}$, from the fit with three templates. The values are not colour corrected. $\sigma_{\text {stat }} \equiv$ statistical uncertainty on the mean dust SED. $c_{v} \equiv$ uncertainties on the inter-calibration [\%] between Planck and WMAP frequencies (Planck Collaboration I 2014; Bennett et al. 2013). $\sigma_{\mathrm{cmb}} \equiv$ uncertainty on the mean dust SED introduced by the CMB subtraction multiplied by the inter-calibration factor $c_{v} . \sigma_{\text {tot }} \equiv$ total uncertainty on the mean dust SED. $\mathrm{S} / \mathrm{N} \equiv$ signal-to-noise ratio on the mean dust SED. $C \equiv$ colour-correction factors computed with a linear combination of the power-law model and the MBB parameters listed in Table 4. $U \equiv$ unit conversion factors from thermodynamic $\left(\mathrm{K}_{\mathrm{CMB}}\right)$ to Rayleigh-Jeans $\left(\mathrm{K}_{\mathrm{RJ}}\right)$ temperature. $\left\langle\left[\tilde{\alpha}_{v}^{I}\right]_{353}^{1 \mathrm{~T}}\right\rangle \equiv$ mean intensity SED in $\mathrm{K}_{\mathrm{RJ}}$ units, normalized to $1 \mathrm{at} 353 \mathrm{GHz}$, derived from the correlation of the maps with the one-template fit. The values are not colour corrected. $\sigma_{\text {tot }}^{1 \mathrm{~T}} \equiv$ total uncertainty on the mean intensity SED with the one-template fit. $\left\langle\left[\tilde{\alpha}_{v}^{I}\right]_{353}^{2 \mathrm{~T}}\right\rangle \equiv$ mean intensity SED in $\mathrm{K}_{\mathrm{RJ}}$ units, normalized to 1 at $353 \mathrm{GHz}$, derived from the correlation of the maps with the two-template fit. The values are not colour corrected. $\sigma_{\text {tot }}^{2 \mathrm{~T}} \equiv$ total uncertainty on the mean intensity SED with the two-template fit.

and warm neutral medium (WNM). In our analysis, we use the predicted SPDUST (v2) spectra (Ali-Haïmoud et al. 2009; Silsbee et al. 2011) of the CNM and WNM spinning dust components. Following the work of Hoang et al. (2011) and Ghosh et al. (2012), we shift both the WNM and CNM spectra in frequency space to fit the observed dust SED. The same DI model of the thermal dust emission is considered for this model. In this case, the spectral model is given in $\mathrm{K}_{\mathrm{RJ}}$ units, normalized to 1 at the frequency $v_{\text {ref }}$, by:

$$
\begin{aligned}
\left\langle\left[\tilde{\alpha}_{v}^{\mathrm{I}}\right]_{353}^{3 \mathrm{~T}}\right\rangle= & A_{\mathrm{WNM}}^{\mathrm{I}} D_{\mathrm{WNM}}\left(v-\Delta v_{\mathrm{WNM}}\right) \\
& +A_{\mathrm{CNM}}^{\mathrm{I}} D_{\mathrm{CNM}}\left(v-\Delta v_{\mathrm{CNM}}\right) \\
& +\left(\frac{v}{v_{\mathrm{ref}}}\right)^{\beta_{\mathrm{d}, \mathrm{mm}}^{\mathrm{I}}-2} \frac{B_{v}\left(T_{\mathrm{d}}\right)}{B_{v_{\mathrm{ref}}}\left(T_{\mathrm{d}}\right)}
\end{aligned}
$$

where $A_{\mathrm{WNM}}^{\mathrm{I}}$ is the amplitude of WNM spectrum normalized at $23 \mathrm{GHz}, A_{\mathrm{CNM}}^{\mathrm{I}}$ is the amplitude of CNM spectrum normalized at $41 \mathrm{GHz}, D_{\mathrm{WNM}}$ is the SPDUST WNM spectrum, $D_{\mathrm{CNM}}$ is the SPDUST CNM spectrum, $\Delta \nu_{\mathrm{WNM}}$ is the shift in the WNM spectrum, $\Delta v_{\mathrm{CNM}}$ is the shift in the CNM spectrum, $v_{\text {ref }}=353 \mathrm{GHz}$ is the reference frequency, $\beta_{\mathrm{d}, \mathrm{mm}}^{\mathrm{I}}$ is the spectral index of the thermal dust emission. We fix $\beta_{\mathrm{d}, \mathrm{mm}}^{\mathrm{I}}=1.51$ from Sect. 6.2 and $T_{\mathrm{d}}=19.6 \mathrm{~K}$ from Sect. 5.2. The four free parameters of the model are the WNM amplitude, the WNM frequency shift, the CNM amplitude and the CNM frequency shift.

The fits of the dust SED with models DI+AI and DI+AII are shown in Fig. 8. The best fit model parameters are listed in Table 4.
Table 4. Results of the spectral fits to the mean dust SED in intensity using Planck and WMAP maps.

\begin{tabular}{lclc}
\hline \hline Parameters & DI+AI & Parameters & DI+AII \\
\hline$\beta_{\mathrm{d}, \mathrm{mm}}^{\mathrm{I}} \cdots$ & $1.52 \pm 0.01$ & $A_{\mathrm{WNM}}^{\mathrm{I}} \cdots$ & $1.12 \pm 0.04$ \\
$A_{\mathrm{a}}^{\mathrm{I}} \ldots \ldots$ & $1.14 \pm 0.04$ & $\Delta \nu_{\mathrm{WNM}}[\mathrm{GHz}]$ & $-1.7 \pm 0.8$ \\
$v_{\mathrm{p}}[\mathrm{GHz}]$ & $9.5 \pm 6.9$ & $A_{\mathrm{CNM}}^{\mathrm{I}} \cdots$ & $0.07 \pm 0.01$ \\
$m_{60} \ldots \ldots$ & $1.81 \pm 0.38$ & $\Delta v_{\mathrm{CNM}}[\mathrm{GHz}]$ & $22.2 \pm 1.6$ \\
$\chi^{2} / N_{\text {d.o.f. }}$. & $2.4 / 8$ & $\chi^{2} / N_{\text {d.o.f. }} \cdot$ & $8.7 / 8$ \\
\hline
\end{tabular}

Notes. The parameters listed in this table are described in Eqs. (26) and (27) where the dust SED is expressed in $\mathrm{K}_{\mathrm{RJ}}$ units and normalized to 1 at $353 \mathrm{GHz}$. The fixed model parameter is $T_{\mathrm{d}}=19.6 \mathrm{~K}$ for two intensity models.

\section{Dust spectral index for polarization}

We now move to the analysis of the polarization data. Like in Sect. 6, we estimate the polarized dust spectral index $\left(\beta_{\mathrm{d}, \mathrm{mm}}^{\mathrm{P}}\right)$ at microwave frequencies $(v \leq 353 \mathrm{GHz})$. We present the results of our data analysis and tests of its robustness against systematic uncertainties.

\subsection{Measuring $\beta_{d, m m}^{P}$}

Here we use the full mission Planck polarization maps, keeping the polarized detector set maps (DS1 and DS2) at $353 \mathrm{GHz}$ as fixed templates, to derive a mean $\beta_{\mathrm{d}, \mathrm{mm}}^{\mathrm{P}}$ using the one-template fit. Using the polarization CC coefficients, $\left[\alpha_{v}^{\mathrm{P}}\right]_{353}^{1 \mathrm{~T}}$, we compute $R_{100}^{\mathrm{P}}(353,217)$ for each sky patch using Eq. $(18)$. Figure 9 shows the values of $R_{100}^{\mathrm{P}}(353,217)$ versus the local dispersion of the 
Planck Collaboration: Frequency dependence of thermal emission from Galactic dust in intensity and polarization
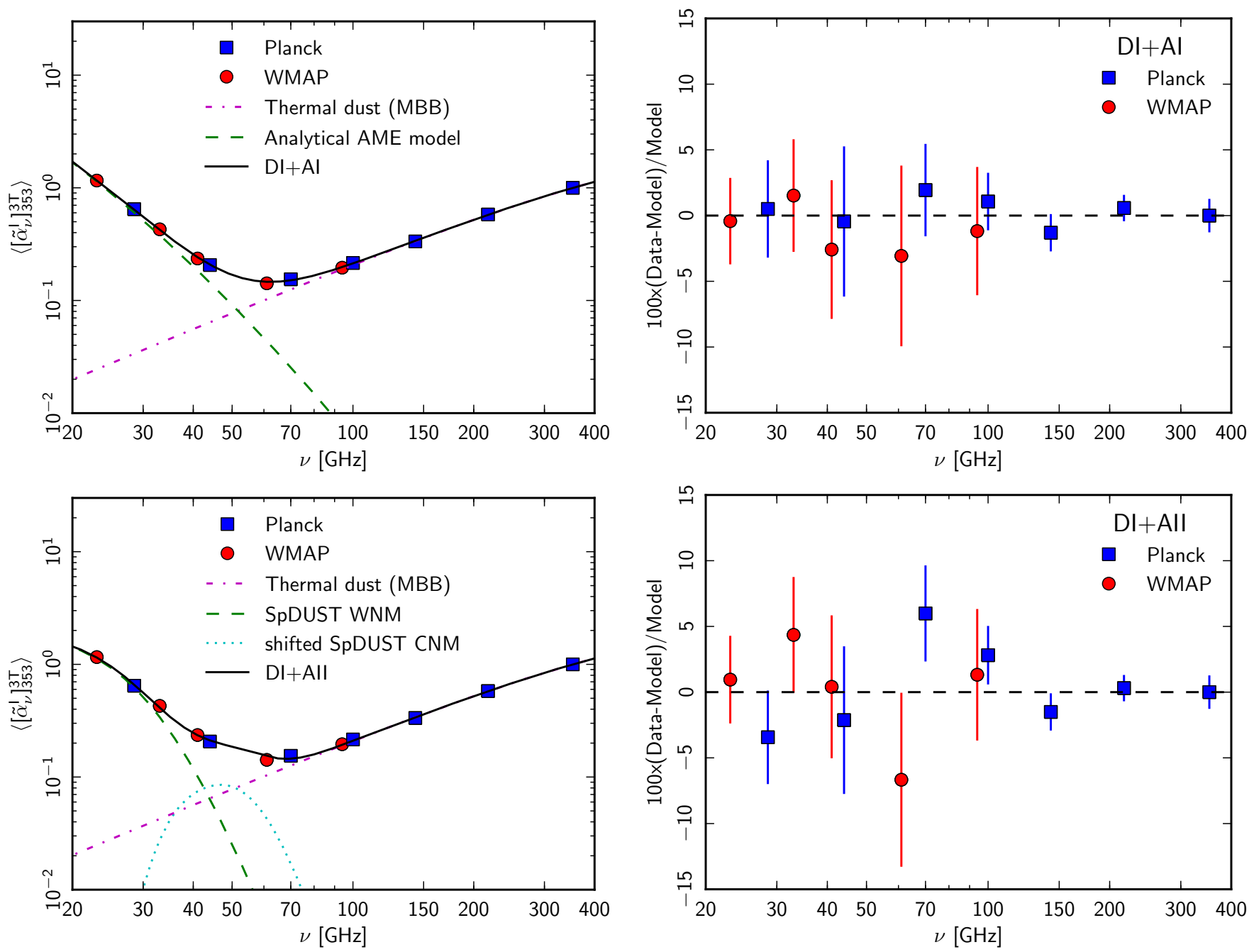

Fig. 8. Mean dust SED in $K_{R J}$ units, normalized to 1 at $353 \mathrm{GHz}$, with different spectral fits and the respective residuals. The two parametric model fits are DI+AI (top left), and DI+AII (bottom left), as presented in Sect. 7.2. Right: residuals after removing the best fit model listed in Table 4, from the mean dust SED. The two spectral models provide good fit to the data, with residuals compatible with zero.

polarized map at $353 \mathrm{GHz}\left(\sigma_{353}^{\mathrm{P}}\right)$ for all the sky patches. To compute $\sigma_{353}^{\mathrm{P}}$, we use a $1^{\circ}$ smoothed map of $P_{353}$ derived in Planck Collaboration Int. XIX (2015). We derive $\beta_{\mathrm{d}, \mathrm{mm}}^{\mathrm{P}}$ for each sky patch from $R_{100}^{\mathrm{P}}(353,217)$, taking into account the local estimate of $T_{\mathrm{d}}$ derived from $R^{\mathrm{I}}(3000,857)$ (Sect. 5.2). We assume that the temperature of the dust grains contributing to the polarization is the same as that determined for the dust emission in intensity. This is not necessarily true if the polarization is associated with specific dust grains, e.g., the silicates versus carbon dust (Martin 2007; Draine \& Fraisse 2009). This should be kept in mind in thinking of physical interpretations. Here we use the spectral indices $\beta_{\mathrm{d}, \mathrm{mm}}^{\mathrm{P}}$ and $\beta_{\mathrm{d}, \mathrm{mm}}^{\mathrm{I}}$ as a mathematical way to quantify the difference between the dust SED for intensity and polarization.

The scatter on the $R_{100}^{\mathrm{P}}(353,217)$ values increases for $\sigma_{353}^{\mathrm{P}}<$ $20 \mu \mathrm{K}$ due to data noise. The histogram of $\beta_{\mathrm{d}, \mathrm{mm}}^{\mathrm{P}}$ from the 400 sky patches is presented in Fig. 9. The distribution of $\beta_{\mathrm{d}, \mathrm{mm}}^{\mathrm{P}}$ has a mean value of 1.592 (round-off to 1.59 ), with a $1 \sigma$ dispersion of 0.174 (round-off to 0.17 ). This dispersion is the same if we use the mean dust temperature of $19.6 \mathrm{~K}$ for all sky patches. The statistical uncertainty on the mean $\beta_{\mathrm{d}, \mathrm{mm}}^{\mathrm{P}}$ is computed from the $1 \sigma$ dispersion divided by the square root of the number of independent sky patches $\left(400 / N_{\text {visit }}\right)$ used, which is 0.02 .
The mean value of the dust spectral index for polarization is different from that for intensity, $1.51 \pm 0.01$ (Sect. 6) over the same sky area. In the next section, we check whether the difference of spectral indices in intensity and polarization is a robust result against systematics present in the polarization data.

\subsection{Uncertainties in $\beta_{d, m m}^{P}$}

For the mean polarized dust spectral index, we use the results from full mission Planck polarization maps with the two detector set maps as fixed templates (Sect. 8.1). To estimate the systematic uncertainty for the mean $\beta_{\mathrm{d}, \mathrm{mm}}^{\mathrm{P}}$, we apply the $\mathrm{CC}$ analysis on multiple subsets of the Planck data, including the combination of yearly maps (YR1 and YR2), the full mission half-ring maps (HR1 and HR2), the combination of odd surveys (S1+S3) and even surveys (S2+S4), and the detector set maps (DS1 and DS2; see Sect. 2.1.2 for more details). We use these subsets of the data as maps and templates at $353 \mathrm{GHz}$. Table 5 lists the derived mean $\beta_{\mathrm{d}, \mathrm{mm}}^{\mathrm{P}}$, for all the sky patches from each combination of the data subsets. The dispersion of the $\beta_{\mathrm{d}, \mathrm{mm}}^{\mathrm{P}}$ values in Table 5, 0.02, is consistent with the $1 \sigma$ dispersion on the mean polarization spectra index from statistical uncertainties estimated in Sect. 8.1, making it difficult to separate the 

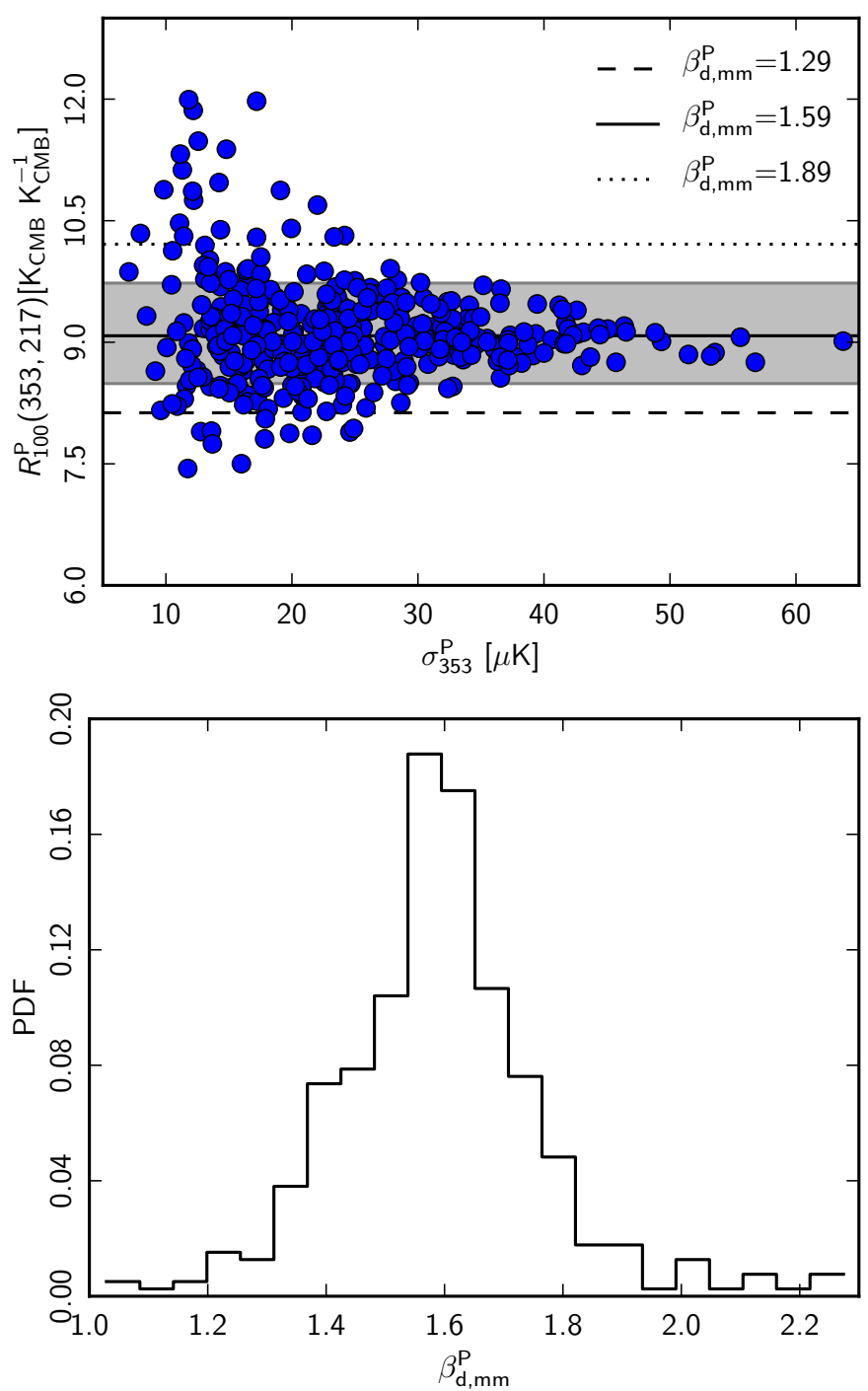

Fig. 9. Top: colour ratio $R_{100}^{\mathrm{P}}(353,217)$ against the local dispersion of the polarization template at $353 \mathrm{GHz}$. Bottom: histogram of the $\beta_{\mathrm{d}, \mathrm{mm}}^{\mathrm{P}}$ values inferred from $R_{100}^{\mathrm{P}}(353,217)$ for all sky patches. The mean value of the spectral index for polarization is $1.59 \pm 0.02$, which is different from that for intensity $1.51 \pm 0.01$ (Fig. 3).

contributions from the statistical noise and the data systematics. Therefore, we use the $1 \sigma$ dispersion from the subsets of the Planck data, as listed in Table 5, as a combine statistical and systematic uncertainties on the mean value of $\beta_{\mathrm{d}, \mathrm{mm}}^{\mathrm{P}}$. Thus, we find $\beta_{\mathrm{d}, \mathrm{mm}}^{\mathrm{P}}=1.59 \pm 0.02$ (stat.+ syst.). The small difference, 0.08 , between $\beta_{\mathrm{d}, \mathrm{mm}}^{\mathrm{P}}$ and $\beta_{\mathrm{d}, \mathrm{mm}}^{\mathrm{I}}$ has a $3.6 \sigma$ significance, taking into account the total uncertainty on both $\beta_{\mathrm{d}, \mathrm{mm}}^{\mathrm{P}}$ and $\beta_{\mathrm{d}, \mathrm{mm}}^{\mathrm{I}}$.

\section{Spectral energy distribution of dust polarization}

We now derive the mean SED for the dust polarization and extend to polarization the parametric modelling already made on the dust SED in intensity (Sect. 7.2).

\subsection{Mean polarized SED}

We use the full mission Planck maps for the spectral modelling of the dust SED in polarization. Like in Sect. 7.1 for dust emission in intensity, the mean SED for polarization is obtained

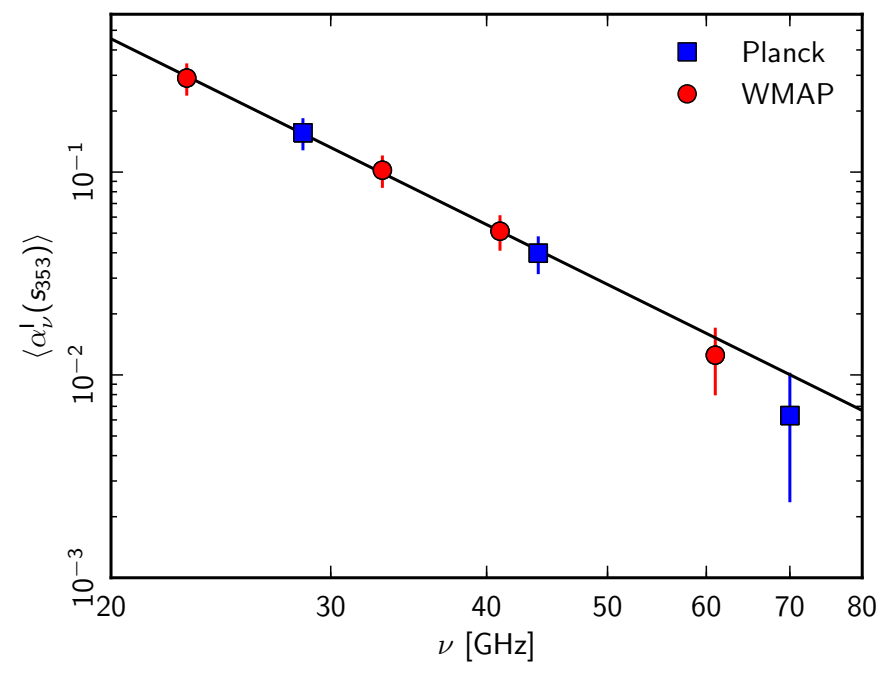

Fig. 10. Spectral energy distribution of the dust-correlated synchrotron emission in intensity. The SED is the ratio between the synchrotron emission at frequency $v$ and the dust emission at $353 \mathrm{GHz}$ emission in units of $\mathrm{K}_{\mathrm{RJ}}$. The mean spectral index for the intensity is $\beta_{\mathrm{s}}^{\mathrm{I}}=$ $-3.04 \pm 0.36$.

Table 5. Polarized dust spectral indices derived using multiple subsets and templates of the Planck data.

\begin{tabular}{|c|c|c|}
\hline Templates & Data sets & $\beta_{\mathrm{d}, \mathrm{mm}}^{\mathrm{P}}$ \\
\hline & Full & 1.592 \\
\hline & HR1 & 1.595 \\
\hline \multirow{3}{*}{ DS1 and DS $2 \ldots$} & HR2 & 1.595 \\
\hline & YR1 & 1.619 \\
\hline & YR2 & 1.592 \\
\hline \multirow{2}{*}{$\ldots \ldots \ldots \ldots$} & Full & 1.602 \\
\hline & HR1 & 1.603 \\
\hline \multirow[t]{3}{*}{ YR1 and YR2 ... } & HR2 & 1.606 \\
\hline & DS1 & 1.564 \\
\hline & DS2 & 1.627 \\
\hline$\ldots$ & Full & 1.613 \\
\hline & DS1 & 1.579 \\
\hline \multirow{2}{*}{$\mathrm{HR} 1$ and $\mathrm{HR} 2 \ldots$} & DS2 & 1.639 \\
\hline & YR1 & 1.639 \\
\hline$\ldots \ldots \ldots \ldots$ & YR2 & 1.614 \\
\hline \multirow[t]{2}{*}{$\ldots \ldots \ldots \ldots \ldots$} & Full & 1.578 \\
\hline & DS1 & 1.560 \\
\hline \multirow{2}{*}{$\mathrm{S} 1+\mathrm{S} 3$ and $\mathrm{S} 2+\mathrm{S} 4$} & DS2 & 1.590 \\
\hline & HR1 & 1.581 \\
\hline$\ldots \ldots \ldots \ldots$ & HR2 & 1.588 \\
\hline
\end{tabular}

Notes. The full mission Planck polarization data along with the DS1 and DS2 templates (first entry in the Table below) is used in Sect. 8.1 to produce Fig. 9 . The scatter of the 20 measurements is consistent with the $1 \sigma$ statistical uncertainty on the mean value of $\beta_{\mathrm{d}, \mathrm{mm}}^{\mathrm{P}}$.

by averaging the polarization $\mathrm{CC}$ coefficients after $\mathrm{CMB}$ subtraction (see Sect. 5.3) over all sky patches and is expressed in $\mathrm{K}_{\mathrm{RJ}}$ units. The polarization SED is derived from the onetemplate fit, keeping the templates fixed to the polarized detector set maps (DS1 and DS2) at $353 \mathrm{GHz}$ (see Sect. 4.3 for more details). We compute the mean polarization SED and its uncertainties in a similar manner to that discussed in Sect. 7. The mean polarized SED and associated uncertainties are listed in Table 6, and is shown in Fig. 11. 
Table 6. Mean microwave SED for polarization computed using the CC analysis.

\begin{tabular}{|c|c|c|c|c|c|c|c|c|c|c|c|c|}
\hline \multirow[b]{2}{*}{ Quantity } & \multicolumn{12}{|c|}{$\begin{array}{c}\text { Frequency [GHz] } \\
\text { Experiment }\end{array}$} \\
\hline & $\begin{array}{l}\text { WMAP } \\
23\end{array}$ & $\begin{array}{c}\text { Planck } \\
28.4\end{array}$ & $\begin{array}{l}\text { WMAP } \\
33\end{array}$ & $\begin{array}{l}\text { WMAP } \\
41\end{array}$ & $\begin{array}{c}\text { Planck } \\
44.1\end{array}$ & $\begin{array}{l}\text { WMAP } \\
61\end{array}$ & $\begin{array}{l}\text { Planck } \\
70.4\end{array}$ & $\begin{array}{l}\text { WMAP } \\
94\end{array}$ & $\begin{array}{c}\text { Planck } \\
100\end{array}$ & $\begin{array}{c}\text { Planck } \\
143\end{array}$ & $\begin{array}{c}\text { Planck } \\
217\end{array}$ & $\begin{array}{c}\text { Planck } \\
353\end{array}$ \\
\hline$\left\langle\left[\tilde{\alpha}_{v}^{\mathrm{P}}\right]_{353}^{1 \mathrm{~T}}\right\rangle$ & 0.9481 & 0.4038 & 0.3351 & 0.1793 & 0.1525 & 0.1179 & 0.1129 & 0.1852 & 0.1900 & 0.3029 & 0.5624 & 1.0000 \\
\hline$\sigma_{\text {stat }} \ldots$ & 0.1201 & 0.0538 & 0.0402 & 0.0292 & 0.0190 & 0.0198 & 0.0118 & 0.0261 & 0.0050 & 0.0048 & 0.0062 & 0.0068 \\
\hline$c_{v}[\%]$ & 1.0 & 1.0 & 1.0 & 1.0 & 1.0 & 1.0 & 0.5 & 1.0 & 0.5 & 0.5 & 0.5 & 1.0 \\
\hline$\sigma_{\mathrm{cmb}}$ & 0.0006 & 0.0006 & 0.0006 & 0.0006 & 0.0006 & 0.0005 & 0.0003 & 0.0005 & 0.0002 & 0.0002 & 0.0001 & 0.0000 \\
\hline$\sigma_{\text {tot }}$ & 0.1204 & 0.0539 & 0.0403 & 0.0293 & 0.0190 & 0.0199 & 0.0118 & 0.0262 & 0.0051 & 0.0050 & 0.0067 & 0.0114 \\
\hline$S / N$ & 7.9 & 7.5 & 8.3 & 6.1 & 8.0 & 5.9 & 9.6 & 7.1 & 37.1 & 60.4 & 83.6 & 87.7 \\
\hline
\end{tabular}

Notes. $\left\langle\left[\tilde{\alpha}_{v}^{P}\right]_{353}^{1 \mathrm{~T}}\right\rangle \equiv$ Mean polarization SED in $\mathrm{K}_{\mathrm{RJ}}$ units, normalized to 1 at $353 \mathrm{GHz}$, from the correlation with the $353 \mathrm{GHz}$ templates. The values are not colour corrected. $\sigma_{\text {stat }} \equiv$ Statistical uncertainty on the mean polarization SED. $c_{v} \equiv$ Uncertainties on the inter-calibration [\%] between Planck and WMAP frequencies (Planck Collaboration I 2014; Bennett et al. 2013). $\sigma_{\mathrm{cmb}} \equiv$ Uncertainty on the mean polarized SED introduced by the CMB-subtraction multiplied by the inter-calibration factor $c_{v} . \sigma_{\text {tot }} \equiv$ Total uncertainty on the mean polarized SED.
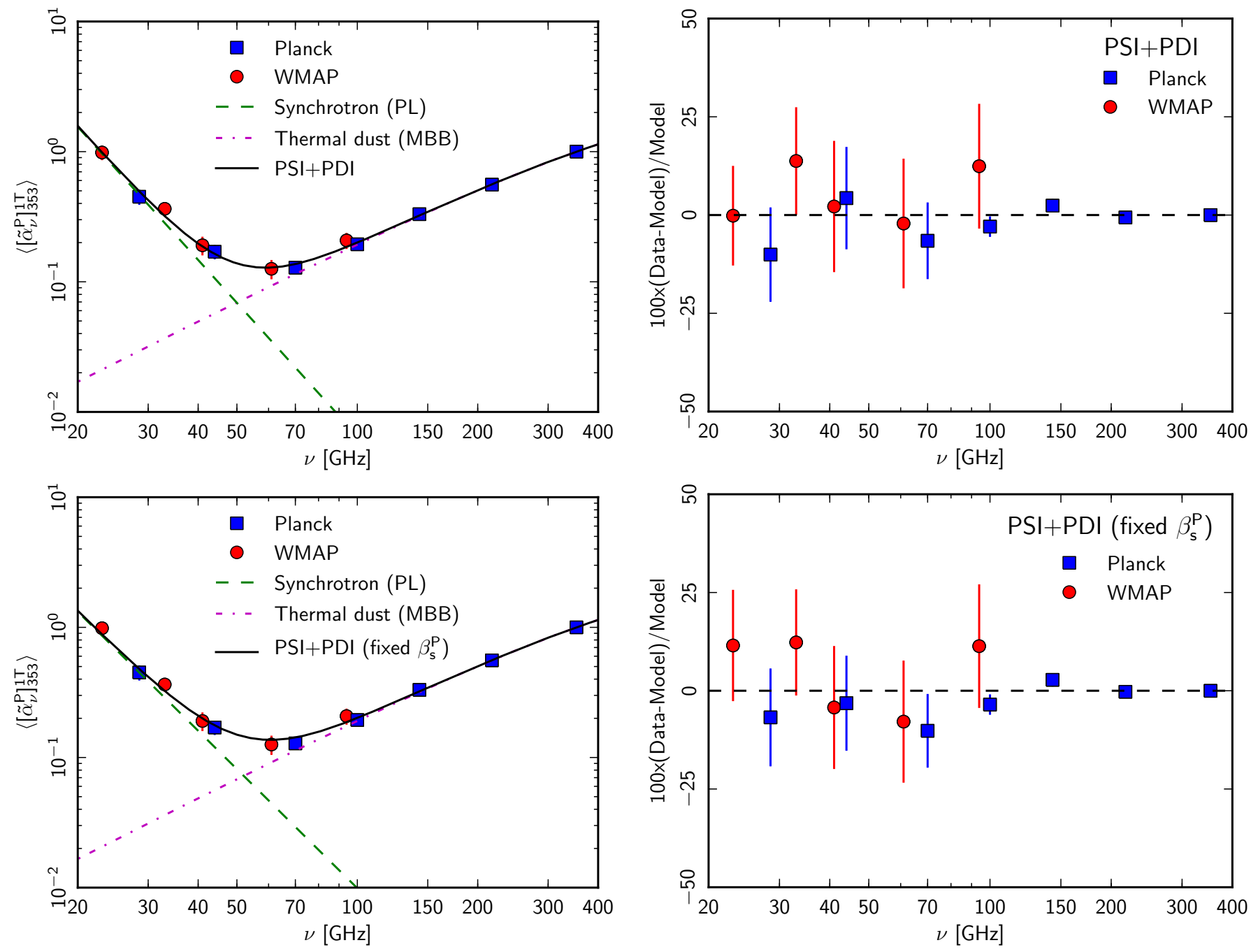

Fig. 11. Mean polarized SED in $\mathrm{K}_{\mathrm{RJ}}$ units, normalized to 1 at $353 \mathrm{GHz}$ correlated with the Stokes $Q$ and $U 353 \mathrm{GHz}$ maps. The polarized spectral model with and without the constraint on $\beta_{\mathrm{s}}^{\mathrm{P}}$ match the observed data points.

The polarization SED first decreases with decreasing frequency, then turns up below $60 \mathrm{GHz}$. This is the first time that such a behavior has been observed for polarized emission correlated with dust polarization, though it has been seen before for the total sky polarization (Bennett et al. 2013).

\subsection{Low frequency rise of the polarization SED}

In this section, we show that a synchrotron component correlated with dust is the most likely interpretation for the low frequency rise of the polarization SED. 


\subsubsection{Synchrotron polarization correlated with dust}

The polarized dust and synchrotron emissions may be written as

$\left[Q_{v}^{\mathrm{s}}, U_{v}^{\mathrm{s}}\right]=p_{\mathrm{s}} I_{v}^{\mathrm{s}}\left[\cos 2 \psi_{\mathrm{s}}, \sin 2 \psi_{\mathrm{s}}\right]$,

$\left[Q_{353}^{\mathrm{d}}, U_{353}^{\mathrm{d}}\right]=p_{\mathrm{d}} I_{353}^{\mathrm{d}}\left[\cos 2 \psi_{\mathrm{d}}, \sin 2 \psi_{\mathrm{d}}\right]$,

where $p_{\mathrm{s}}$ and $p_{\mathrm{d}}$ are the polarization fractions, and $\psi_{\mathrm{s}}$ and $\psi_{\mathrm{d}}$ are the polarization angles, for synchrotron and dust, respectively. After correlation with the $353 \mathrm{GHz} I, Q$ and $U$ templates, we have

$\left\langle\alpha_{v}^{\mathrm{P}}\left(s_{353}\right)\right\rangle \leq \frac{p_{\mathrm{s}}}{p_{\mathrm{d}}}\left\langle\alpha_{v}^{\mathrm{I}}\left(s_{353}\right)\right\rangle$,

where $\left\langle\alpha_{v}^{\mathrm{I}}\left(s_{353}\right)\right\rangle$ and $\left\langle\alpha_{v}^{\mathrm{P}}\left(s_{353}\right)\right\rangle$ are the mean SEDs of the synchrotron emission correlated with dust in intensity and polarization. The upper limit in Eq. (30) is obtained when the synchrotron and dust polarization angles are identical, which is not what is observed comparing the Planck $353 \mathrm{GHz}$ and WMAP $23 \mathrm{GHz}$ polarization data (Planck Collaboration Int. XIX 2015). Both emission processes trace the same large-scale Galactic magnetic field (GMF), but they give different weights to different parts of the line of sight. The CC analysis only keeps the synchrotron emission that arises from the same volume of interstellar space as the dust emission. For example, it is expected to filter out the synchrotron emission from the Galactic halo, where there is little dust. Thus, to validate our interpretation of the low frequency rise of the polarization SED with synchrotron and no AME polarization, we need to show that the upper limit in Eq. (30) holds.

The intrinsic polarization of synchrotron emission is about $75 \%$ for typical relativistic electron spectra (Rybicki \& Lightman 1979; Longair 1994), whereas the analysis of Planck polarization maps indicates that the intrinsic polarization of dust at $353 \mathrm{GHz}$ can reach about $20 \%$ (Planck Collaboration Int. XIX 2015; Planck Collaboration Int. XX 2015). To compute the synchrotron SED in intensity, $\left\langle\alpha_{v}^{\mathrm{I}}\left(s_{353}\right)\right\rangle$, we combine the $353 \mathrm{GHz}$ correlated CC coefficients, corrected for the CMB (Sect. 5.3), obtained from the fits with one and two templates (Sect. 4.2). The SED of the synchrotron emission correlated with dust is then obtained by taking the difference between the CC coefficients in Eqs. (8) and (11), and averaging over all sky patches. It is shown in Fig. 10. We fit this synchrotron SED with a powerlaw (PL) model. The normalized amplitude of synchrotron emission at $23 \mathrm{GHz}$ is $A_{\mathrm{s}}^{\mathrm{I}}=0.30$. This is the ratio between the 23 and $353 \mathrm{GHz}$ emission in units of $\mathrm{K}_{\mathrm{RJ}}$. The mean synchrotron spectral index derived from the fit is $\beta_{\mathrm{s}}^{\mathrm{I}}=-3.04 \pm 0.36$. The uncertainty on $\beta_{\mathrm{s}}$ is overestimated as the uncertainties on the synchrotron SED are highly correlated across all WMAP and Planck frequencies. However, this is not critical for our study because we do not use the uncertainty on $\beta_{\mathrm{s}}$ in the paper. The derived mean $\beta_{\mathrm{s}}^{\mathrm{I}}$ of the dust-correlated synchrotron emission is consistent with the spectral index of 408-MHz-correlated synchrotron emission obtained using WMAP data (Miville-Deschênes et al. 2008; Dickinson et al. 2009; Gold et al. 2011; Ghosh et al. 2012), and the spectral index of the polarized synchrotron emission (Fuskeland et al. 2014).

Using $\left\langle\alpha_{23}^{\mathrm{I}}\left(s_{353}\right)\right\rangle$, we find the theoretical upper limit on $\left\langle\alpha_{23}^{\mathrm{P}}\left(s_{353}\right)\right\rangle$ to be

$\left\langle\alpha_{23}^{\mathrm{P}}\left(s_{353}\right)\right\rangle \leq\left(\frac{0.75}{0.20}\right) \times 0.30=1.1$.

The measured value of $\left\langle\left[\tilde{\alpha}_{v}^{\mathrm{P}}\right]_{353}^{1 \mathrm{~T}}\right\rangle$ is 0.95 (shown in Fig. 11), which is within the upper limit. The difference between the measured and theoretical upper limit in Eq. (30) can be explained by the fact that polarization angles traced by synchrotron and dust emission are not perfectly aligned. We point out that that statement refers to the synchrotron emission correlated with the dust template in intensity, which is not one to one correlated with dust in polarization.

\subsubsection{Upper limit on AME polarization}

We can set an upper limit on the polarization fraction of AME by assuming that the synchrotron and dust polarization are totally uncorrelated. Within this hypothesis, the low frequency rise of the polarization SED is entirely due to polarized AME. Since both AME and thermal dust emission are associated with interstellar matter, it is reasonable to assume that the polarization angles are the same for AME and dust. We obtain the $353 \mathrm{GHz}$ correlated AME polarization at $23 \mathrm{GHz}$ as,

$$
\begin{aligned}
& \left\langle\left[\tilde{\alpha}_{23}^{\mathrm{P}}\right]_{353}^{1 \mathrm{~T}}\right\rangle=\left\langle\alpha_{23}^{\mathrm{P}}\left(a_{353}\right)\right\rangle=\frac{p_{\mathrm{a}}}{p_{\mathrm{d}}}\left\langle\alpha_{23}^{\mathrm{I}}\left(a_{353}\right)\right\rangle \\
& \text { i.e., } 0.95=\frac{p_{\mathrm{a}}}{p_{\mathrm{d}}}\left\langle\alpha_{23}^{\mathrm{I}}\left(a_{353}\right)\right\rangle \\
& \text { so } p_{\mathrm{a}}=\frac{p_{\mathrm{d}} \times 0.95}{\left\langle\alpha_{23}^{\mathrm{I}}\left(a_{353}\right)\right\rangle} \\
& =\frac{p_{\mathrm{d}} \times 0.95}{A_{\mathrm{a}}^{\mathrm{I}}}
\end{aligned}
$$

We use the mean AME amplitude, $A_{\mathrm{a}}^{\mathrm{I}}=1.14$, from model DI+AI and Table 4, together with $p_{\mathrm{d}}=20 \%$ (Planck Collaboration Int. XIX 2015; Planck Collaboration Int. XX 2015), to derive an upper limit on the intrinsic polarization fraction of AME of about $16 \%$. This is much higher than upper limits reported from the analysis of compact sources (Dickinson et al. 2011; López-Caraballo et al. 2011; Rubiño-Martín et al. 2012) and theoretical predictions (Lazarian \& Draine 2000; Hoang et al. 2013). Thus AME is unlikely to be the sole explanation for the low frequency rise of the polarization SED, even if we cannot exclude some contribution from AME.

\subsection{Parametric modelling for polarized dust SED}

In this section we present a spectral model that fits the observed polarization SED. We model the polarization SED with a combination of polarized synchrotron and dust components. This model does not include AME. We account for the rise of the SED towards the lowest frequencies with the synchrotron component. For the synchrotron component we use the PL model with two parameters: the amplitude; and the spectral index. The PL model of synchrotron emission is related to the power-law energy distribution of the cosmic-ray electron spectrum (Abdo et al. 2009; Ackermann et al. 2010, 2012). The model is the superposition of a power-law synchrotron spectrum and the MBB for the thermal dust emission. We refer to this model as PSI+PDI. It is described by the equation:

$\left\langle\left[\tilde{\alpha}_{v}^{\mathrm{P}}\right]_{353}^{1 \mathrm{~T}}\right\rangle=A_{\mathrm{s}}^{\mathrm{P}}\left(\frac{v}{v_{\mathrm{b}}}\right)^{\beta_{\mathrm{s}}^{\mathrm{P}}}+\left(\frac{v}{v_{\text {ref }}}\right)^{\beta_{\mathrm{d}, \mathrm{mm}}^{\mathrm{P}}-2} \frac{B_{v}\left(T_{\mathrm{d}}\right)}{B_{v_{\text {ref }}}\left(T_{\mathrm{d}}\right)}$,

where $A_{\mathrm{s}}^{\mathrm{P}}$ is the amplitude of polarized synchrotron components in $\mathrm{K}_{\mathrm{RJ}}$ units, and $\beta_{\mathrm{d}, \mathrm{mm}}^{\mathrm{P}}$ is the polarized dust spectral index. The polarized dust SED, expressed in $\mathrm{K}_{\mathrm{RJ}}$ units, is normalized to 1 at $353 \mathrm{GHz}$. Like for the two intensity models, we fix $T_{\mathrm{d}}=19.6 \mathrm{~K}$. We fit three parameters: the synchrotron amplitude, 
Planck Collaboration: Frequency dependence of thermal emission from Galactic dust in intensity and polarization

Table 7. Results of the spectral fits to the mean polarized dust SED obtained using Planck and WMAP data.

\begin{tabular}{ccc}
\hline \hline Parameters $^{a}$ & Unconstrained $\beta_{\mathrm{s}}^{\mathrm{P}}$ & Fixed $\beta_{\mathrm{s}}^{\mathrm{P}}$ \\
\hline$A_{\mathrm{s}}^{\mathrm{P}} \cdots \cdots$ & $0.97 \pm 0.10$ & $0.86 \pm 0.06$ \\
$\beta_{\mathrm{s}}^{\mathrm{P}} \cdots \cdots$ & $-3.40 \pm 0.28$ & -3.04 \\
$\beta_{\mathrm{d}, \mathrm{mm}}^{\mathrm{P}} \cdots$ & $1.57 \pm 0.01$ & $1.58 \pm 0.01$ \\
$\chi^{2} / N_{\text {dof }} \cdots$ & $6.6 / 9$ & $8.6 / 10$ \\
\hline
\end{tabular}

Notes. ${ }^{(a)}$ The parameters of the model PSI+PDI are described in Eq. (33) for fixed $T_{\mathrm{d}}=19.6 \mathrm{~K}$, where the dust $\mathrm{SED}$ is expressed in $\mathrm{K}_{\mathrm{RJ}}$ units and normalized to 1 at $353 \mathrm{GHz}$.

the synchrotron and dust spectral index. We also fit this model with an additional constraint that the spectral index of $353 \mathrm{GHz}$ correlated synchrotron component is the same for intensity and polarization. By doing so we have one less parameter to fit and increase the number of degrees of freedom by one.

The fits to the polarization SED for model with and without the constraint on $\beta_{\mathrm{s}}^{\mathrm{P}}$ are shown in Fig. 11. The parameters for the best-fit models are listed in Table 7. The two models provide very similar fits to the observed polarized SED. If we force the spectral indices of the synchrotron for intensity and polarization to be equal, we find an equally good fit to the polarization SED. These results are further discussed in the next section.

\section{Comparison of the dust SEDs for intensity and polarization}

We now compare the dust SEDs for intensity and polarization and discuss the frequency dependence of the polarization fraction within the context of existing dust models.

\subsection{Spectral dependence of the polarization fraction}

Figure 12 presents the dust SEDs for intensity and polarization derived from the spectral decomposition in emission components (Sects. 7.2 and 9.3). The SED for intensity, obtained after AME subtraction, is shown in the top panel of Fig. 12 from 70 to $353 \mathrm{GHz}$. The two sets of data points computed for each of the two AME models are very close to each other. The dust SED for polarization, obtained after subtraction of the synchrotron component, is shown in the middle panel of Fig. 12. The data points are plotted for the two spectral indices in Table 7, which differ by 0.4 . The small difference between the corresponding points shows that the subtraction of the synchrotron component has a very small impact on the dust polarization SED even at $70 \mathrm{GHz}$. This indicates that our dust polarization SED is robust with respect to uncertainties on the spectral index of polarized synchrotron, including a possible steepening of the spectrum with increasing frequencies as discussed in Gold et al. (2011). The polarization fraction, shown in the bottom panel of Fig. 12, is normalized with respect to the data point at $353 \mathrm{GHz}$. The uncertainties on $p$ include the uncertainties from both $P$ and $I$. The data suggest that there is a small decrease in $p$ by $21 \pm 6 \%$ from 353 to $70 \mathrm{GHz}$.

\subsection{The contribution of carbon dust and silicates to polarization}

The results of this work set new observational constraints on dust models including silicate and carbon grains with possibly
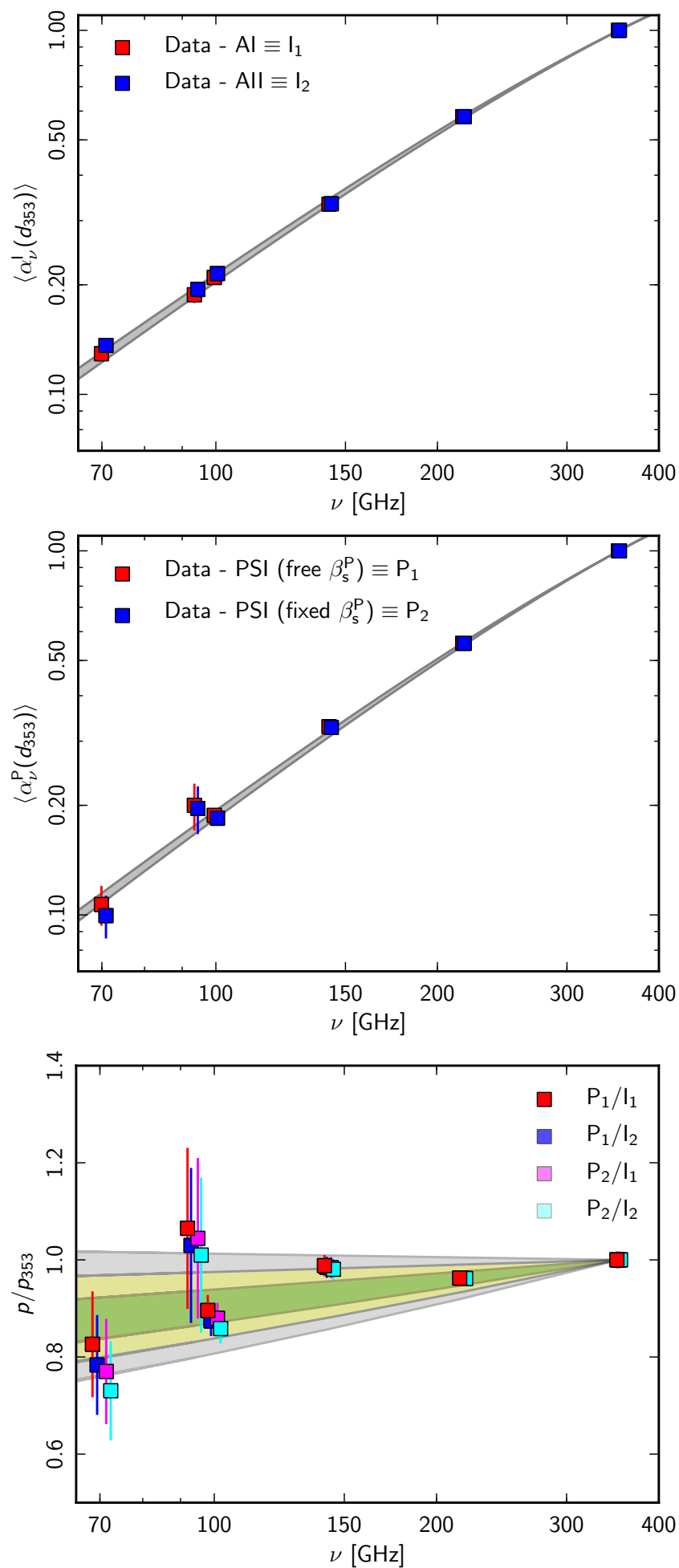

Fig. 12. Frequency dependence of the dust SED in intensity without AME (top), in polarization without synchrotron (middle), and the polarization fraction (bottom) for the four different combinations of dust models. The SEDs are plotted in units of $\mathrm{K}_{\mathrm{RJ}}$ and normalized to 1 at $353 \mathrm{GHz}$. The shaded areas represent $1 \sigma$ (green), $2 \sigma$ (yellow), and $3 \sigma$ (grey) statistical uncertainty on the mean normalized polarization fraction. We used the mean spectra from the same 400 sky patches for both the intensity and polarization analysis.

different polarization properties (Hildebrand et al. 1999; Martin 2007). We discuss the spectral dependence of $p$ using the models from Draine \& Li (2007), Compiègne et al. (2011), and Jones et al. (2013). In these three models, the thermal dust emission 
is the electric dipole emission from two types of grains, silicates and carbon grains, with distinct optical properties and thereby temperatures. The first two models use the same optical properties for silicates, but distinct properties for carbon grains; Draine \& Li (2007) use the optical properties of graphite, while Compiègne et al. (2011) use results from laboratory measurements of amorphous carbon. The spectral index for carbon grains is 2 in the Draine \& Li (2007) model and 1.6 in Compiègne et al. (2011). Over microwave frequencies, the opacity of silicates scales as $v^{1.6}$ in both models. Jones et al. (2013) use optical properties of amorphous carbon grains, which depend on the hydrogen fraction and degree of aromatization (Jones 2012). The spectral index of the carbon dust at $353 \mathrm{GHz}$ varies between 1.2 and 2.3, depending on the nature of the carbon grains (see Fig. 14 of Jones et al. 2013, for more details).

SEDs from the first two models have been compared to the Planck dust SED in intensity in Planck Collaboration Int. XVII (2014) and Planck Collaboration Int. XXIX (2014). The differences between the model and the data are within $5-15 \%$ at $v<353 \mathrm{GHz}$. Draine \& Fraisse (2009) have used the Draine \& Li (2007) dust model to compute spectra for dust polarization. They predict a systematic increase of the polarization fraction $p$ at microwave frequencies when only silicates contribute to dust polarization. This prediction is not what we report in this paper. However, model predictions for the spectral dependency of $p$ are related to the difference in spectral index between carbon and silicate grains, which is not known. Thus, a difference between carbon and silicate polarization may be the correct physical interpretation of the spectral dependence of $p$, even if the data do not match the Draine \& Fraisse (2009) model.

Calculations of the polarized SED for the Compiègne et al. (2011) and Jones et al. (2013) models are needed to assess quantitatively this interpretation. In the Compiègne et al. (2011) model, the spectra from silicates and carbon grains are very similar at long wavelengths and we do not expect $p$ to depend on wavelength when only silicates contribute to the polarization. In the Jones et al. (2013) model the contribution from carbon dust grains could be dominant at $v<353 \mathrm{GHz}$.

\subsection{Microwave dust emission}

The dust SED in polarization and the spectral dependence of $p$ allows us to discuss two possible interpretations of the dust microwave emission.

Meny et al. (2007) introduced a physical description of FIR/microwave dust emission, where the microwave dust opacity of amorphous grains is dominated by low energy transitions associated with disorder in the structure of the solids on atomic scales. This contribution is modelled by transitions in two-level systems (TLS). The TLS model is supported by experimental results on silicates (Agladze et al. 1996; Boudet et al. 2005; Coupeaud et al. 2011), which indicate that the opacity of amorphous silicate grains flattens towards long wavelengths. The TLS model has been used to model dust emission spectra by Paradis et al. (2011). It was also proposed as a possible interpretation of the flattening of the dust SED in intensity from FIR to mm wavelengths, which was reported in two earlier Planck papers on the diffuse dust emission in the Galactic plane in Planck Collaboration Int. XIV (2014) and at high Galactic latitudes in Planck Collaboration Int. XVII (2014). Our analysis based on new Planck data does not confirm this flattening, but does not dismiss it either because the error-bars on the submm spectral index from calibration uncertainties remain significant: $0.16(1 \sigma)$ from Sect. 5.2. Within the TLS model, a flattening of the dust
SED in intensity would be due to a decrease in the spectral index of silicate grains at long wavelengths. Since silicate grains are polarized, the flattening of the dust SED should also be seen in polarization. This expectation is not supported by the results of our data analysis, namely the difference in spectral indices of the thermal dust emission in intensity and polarization $\left(\beta_{\mathrm{d}, \mathrm{mm}}^{\mathrm{I}}\right.$ and $\beta_{\mathrm{d}, \mathrm{mm}}^{\mathrm{P}}$ ) reported in Sect. 8.2.

MDE has been introduced by Draine \& Hensley (2012) to explain the flattening of the dust SED at sub-mm wavelengths in the Small Magellanic Cloud (Planck Collaboration XVII 2011). MDE could also contribute to the long wavelength emission of Galactic dust. Model spectra of MDE are presented in Draine \& Hensley (2013) for Galactic dust. The contribution of MDE could be significant at frequencies smaller than a few hundred $\mathrm{GHz}$ and increasing towards smaller frequencies. If the magnetic particles are inclusions randomly-oriented within interstellar grains, their emission is polarized in a direction perpendicular to that of the dipolar electric emission (Draine \& Hensley 2013). In this case, we expect this emission component to reduce the polarization of the dust emission. This could possibly account for the observed decrease in $p$ from 353 to $70 \mathrm{GHz}$.

A fit of the SEDs in polarization and intensity with the models of Paradis et al. (2011) and Draine \& Hensley (2013) would be necessary to test these two interpretations quantitatively.

\section{Conclusion}

We have characterized the frequency dependence of dust emission in intensity and polarization by analysing Planck data over $39 \%$ of the sky at intermediate Galactic latitudes. We use the Planck $353 \mathrm{GHz} I, Q$, and $U$ maps as templates for dust emission in intensity and polarization. We cross-correlate them with the Planck and WMAP data, at 12 frequencies from 23 to $353 \mathrm{GHz}$. The main results of the data analysis are as follows.

- The mean spectral index of the dust emission measured between 100 and $353 \mathrm{GHz}$ is $\beta_{\mathrm{d}, \mathrm{mm}}^{\mathrm{I}}=1.51 \pm 0.01$. This value agrees with that reported by Planck Collaboration Int. XVII (2014) for the high Galactic latitude sky and Planck Collaboration Int. XIV (2014) for diffuse emission in the Galactic plane. The microwave spectral index $\beta_{\mathrm{d}, \mathrm{mm}}^{\mathrm{I}}$ is close to that at submm wavelengths, which is derived from fits to the Planck data at $v \geq 353 \mathrm{GHz}$ with the full-mission Planck data.

- We determine the mean dust SED in intensity from 23 to $353 \mathrm{GHz}$. We separate the dust and AME contributions to the SED. The dust contribution is well fit by combining the modified blackbody spectrum with a spectral index of 1.51 with the mean temperature of $19.6 \mathrm{~K}$. The two parametric models we use for the AME yield the same SED for the dust for frequencies $v \geq 70 \mathrm{GHz}$.

- The mean spectral index for dust polarization, measured between 100 and $353 \mathrm{GHz}$, is $\beta_{\mathrm{d}, \mathrm{mm}}^{\mathrm{P}}=1.59 \pm 0.02$, assuming the temperature of aligned dust grains contributing to the polarization is the same as that determined from the dust emission in intensity. We show that the small difference with $\beta_{\mathrm{d}, \mathrm{mm}}^{\mathrm{I}}$, the spectral index measured in a similar way for dust intensity, is a robust result against systematic uncertainties estimated comparing results of our data analysis obtained on various subsets of the Planck data.

- We determine the SED of the dust-correlated polarized emission from 23 to $353 \mathrm{GHz}$. This SED decreases with decreasing frequency and turns up below $60 \mathrm{GHz}$, very much like 
the dust SED in intensity due to AME. We show that the low frequency rise of the polarization SED may be explained by synchrotron polarization correlated with dust.

- We use a parametric model to separate the synchrotron and dust polarization and to characterize the spectral dependence of the dust polarization fraction. The polarization fraction $p$ of the dust emission decreases by $(21 \pm 6) \%$ from 353 to $70 \mathrm{GHz}$. We discuss this result within the context of existing dust models. It could indicate differences in polarization efficiency among components of interstellar dust (e.g., carbon and silicate grains).

Our observational results provide inputs to quantify and optimize the separation between Galactic and CMB polarization (Planck Collaboration Int. XXX 2015). Our CC analysis yields a spectral decomposition of the diffuse emission into its main components: thermal emission from dust; free-free; synchrotron; and AME. This spectral decomposition may be combined with power spectra of the templates, as discussed in Appendix D, to quantify the Galactic emission in intensity as a function of the observed frequency and multipole (see Figs. 27 and 28 in Planck Collaboration I 2014). For polarization, we are currently missing a synchrotron polarization template that is free from Faraday rotation. Polarized synchrotron templates, which are expected from C-BASS (King et al. 2010) at $5 \mathrm{GHz}$ and QUIJOTE (Hoyland et al. 2012) between 10 and $20 \mathrm{GHz}$, will be important for future correlation analyses.

Acknowledgements. The Planck Collaboration acknowledges the support of: ESA; CNES and CNRS/INSU-IN2P3-INP (France); ASI, CNR, and INAF (Italy); NASA and DoE (USA); STFC and UKSA (UK); CSIC, MICINN and J.A. (Spain); Tekes, AoF and CSC (Finland); DLR and MPG (Germany); CSA (Canada); DTU Space (Denmark); SER/SSO (Switzerland); RCN (Norway); SFI (Ireland); FCT/MCTES (Portugal); and DEISA (EU). A detailed description of the Planck Collaboration and a list of its members can be found at http://www.rssd.esa.int/index.php?project=PLANCK\&page= Planck_Collaboration. The research leading to these results has received funding from the European Research Council under the European Union's Seventh Framework Programme (FP7/2007-2013)/ERC grant agreement No. 267934. We acknowledge the use of the Legacy Archive for Microwave Background Data Analysis (LAMBDA), part of the High Energy Astrophysics Science Archive Center (HEASARC). HEASARC/LAMBDA is a service of the Astrophysics Science Division at the NASA Goddard Space Flight Center. Some of the results in this paper have been derived using the HEALPix package.

\section{References}

Abdo, A. A., Ackermann, M., Ajello, M., et al. 2009, Phys. Rev. Lett., 102, 181101

Ackermann, M., Ajello, M., Atwood, W. B., et al. 2010, Phys. Rev. D, 82, 092004 Ackermann, M., Ajello, M., Allafort, A., et al. 2012, Phys. Rev. Lett., 108, 011103

Agladze, N. I., Sievers, A. J., Jones, S. A., Burlitch, J. M., \& Beckwith, S. V. W. 1996, ApJ, 462, 1026

Ali-Haïmoud, Y., Hirata, C. M., \& Dickinson, C. 2009, MNRAS, 395, 1055

Banday, A. J., Gorski, K. M., Bennett, C. L., et al. 1996, ApJ, 468, L85

Banday, A. J., Dickinson, C., Davies, R. D., Davis, R. J., \& Górski, K. M. 2003 , MNRAS, 345, 897

Bennett, C. L., Larson, D., Weiland, J. L., et al. 2013, ApJS, 208, 20

Bonaldi, A., Ricciardi, S., Leach, S., et al. 2007, MNRAS, 382, 1791

Boudet, N., Mutschke, H., Nayral, C., et al. 2005, ApJ, 633, 272

Brandt, T. D., \& Draine, B. T. 2012, ApJ, 744, 129

Chiar, J. E., Adamson, A. J., Whittet, D. C. B., et al. 2006, ApJ, 651, 268

Chon, G., Challinor, A., Prunet, S., Hivon, E., \& Szapudi, I. 2004, MNRAS, 350, 914

Compiègne, M., Verstraete, L., Jones, A., et al. 2011, A\&A, 525, A103

Coupeaud, A., Demyk, K., Meny, C., et al. 2011, A\&A, 535, A124

Davies, R. D., Dickinson, C., Banday, A. J., et al. 2006, MNRAS, 370, 1125

de Oliveira-Costa, A., Tegmark, M., Gutierrez, C. M., et al. 1999, ApJ, 527, L9

Dickinson, C., Davies, R. D., \& Davis, R. J. 2003, MNRAS, 341, 369

Dickinson, C., Eriksen, H. K., Banday, A. J., et al. 2009, ApJ, 705, 1607

Dickinson, C., Peel, M., \& Vidal, M. 2011, MNRAS, 418, L35

Dobler, G., \& Finkbeiner, D. P. 2008, ApJ, 680, 1222
Draine, B. T., \& Fraisse, A. A. 2009, ApJ, 696, 1

Draine, B. T., \& Hensley, B. 2012, ApJ, 757, 103

Draine, B. T., \& Hensley, B. 2013, ApJ, 765, 159

Draine, B. T., \& Lazarian, A. 1998, ApJ, 508, 157

Draine, B. T., \& Lazarian, A. 1999, in BAAS, 31, 890

Draine, B. T., \& Li, A. 2007, ApJ, 657, 810

Erickson, W. C. 1957, ApJ, 126, 480

Finkbeiner, D. P., Davis, M., \& Schlegel, D. J. 1999, ApJ, 524, 867

Fuskeland, U., Wehus, I. K., Eriksen, H. K., \& Næss, S. K. 2014, ApJ, 790, 104

Gardner, F. F., \& Whiteoak, J. B. 1966, ARA\&A, 4, 245

Ghosh, T., Banday, A. J., Jaffe, T., et al. 2012, MNRAS, 422, 3617

Gold, B., Odegard, N., Weiland, J. L., et al. 2011, ApJS, 192, 15

Gorski, K. M., Banday, A. J., Bennett, C. L., et al. 1996, ApJ, 464, L11

Górski, K. M., Hivon, E., Banday, A. J., et al. 2005, ApJ, 622, 759

Haslam, C. G. T., Salter, C. J., Stoffel, H., \& Wilson, W. E. 1982, A\&AS, 47, 1

Hauser, M. G., Arendt, R. G., Kelsall, T., et al. 1998, ApJ, 508, 25

Hildebrand, R. H., Dotson, J. L., Dowell, C. D., Schleuning, D. A., \& Vaillancourt, J. E. 1999, ApJ, 516, 834

Hoang, T., Lazarian, A., \& Draine, B. T. 2011, ApJ, 741, 87

Hoang, T., Lazarian, A., \& Martin, P. G. 2013, ApJ, 779, 152

Hoyland, R. J., Aguiar-González, M., Aja, B., et al. 2012, in SPIE Conf. Ser., 8452

Jones, A. P. 2012, A\&A, 542, A98

Jones, A. P., Fanciullo, L., Köhler, M., et al. 2013, A\&A, 558, A62

Kalberla, P. M. W., Burton, W. B., Hartmann, D., et al. 2005, A\&A, 440, 775

King, O. G., Copley, C., Davies, R., et al. 2010, in SPIE Conf. Ser., 7741

Kogut, A., Banday, A. J., Bennett, C. L., et al. 1996, ApJ, 464, L5

Kogut, A., Dunkley, J., Bennett, C. L., et al. 2007, ApJ, 665, 355

Lagache, G. 2003, A\&A, 405, 813

Lazarian, A., \& Draine, B. T. 2000, ApJ, 536, L15

Lehtinen, K., Juvela, M., \& Mattila, K. 2010, A\&A, 517, A79

Leitch, E. M., Readhead, A. C. S., Pearson, T. J., \& Myers, S. T. 1997, ApJ, 486, L23

Liu, H., Mertsch, P., \& Sarkar, S. 2014, ApJ, 789, L29

Longair, M. S. 1994, High energy astrophysics, Vol. 2, Stars, the Galaxy and the interstellar medium (Cambridge University Press)

López-Caraballo, C. H., Rubiño-Martín, J. A., Rebolo, R., \& Génova-Santos, R. 2011, ApJ, 729, 25

Macellari, N., Pierpaoli, E., Dickinson, C., \& Vaillancourt, J. E. 2011, MNRAS, 418, 888

Martin, P. G. 2007, in EAS Pub. Ser. 23, eds. M.-A. Miville-Deschênes, \& F. Boulanger, 165

Mennella, A., Butler, R. C., Curto, A., et al. 2011, A\&A, 536, A3

Meny, C., Gromov, V., Boudet, N., et al. 2007, A\&A, 468, 171

Miville-Deschênes, M.-A., Ysard, N., Lavabre, A., et al. 2008, A\&A, 490, 1093

Page, L., Hinshaw, G., Komatsu, E., et al. 2007, ApJS, 170, 335

Paradis, D., Bernard, J.-P., Mény, C., \& Gromov, V. 2011, A\&A, 534, A118

Planck Collaboration I. 2011, A\&A, 536, A1

Planck Collaboration XVII. 2011, A\&A, 536, A17

Planck Collaboration XX. 2011, A\&A, 536, A20

Planck Collaboration XXIV. 2011, A\&A, 536, A24

Planck Collaboration XXV. 2011, A\&A, 536, A25

Planck Collaboration I. 2014, A\&A, 571, A1

Planck Collaboration II. 2014, A\&A, 571, A2

Planck Collaboration III. 2014, A\&A, 571, A3

Planck Collaboration IV. 2014, A\&A, 571, A4

Planck Collaboration VI. 2014, A\&A, 571, A6

Planck Collaboration VII. 2014, A\&A, 571, A7

Planck Collaboration VIII. 2014, A\&A, 571, A8

Planck Collaboration IX. 2014, A\&A, 571, A9

Planck Collaboration X. 2014, A\&A, 571, A10

Planck Collaboration XI. 2014, A\&A, 571, A11

Planck Collaboration XII. 2014, A\&A, 571, A12

Planck Collaboration XIII. 2014, A\&A, 571, A13

Planck Collaboration XIV. 2014, A\&A, 571, A14

Planck Collaboration XV. 2014, A\&A, 571, A15

Planck Collaboration XVI. 2014, A\&A, 571, A16

Planck Collaboration XXX. 2014, A\&A, 571, A30

Planck Collaboration Int. IX. 2013, A\&A, 554, A139

Planck Collaboration Int. XII. 2013, A\&A, 557, A53

Planck Collaboration Int. XIV. 2014, A\&A, 564, A45

Planck Collaboration Int. XV. 2014, A\&A, 565, A103

Planck Collaboration Int. XVII. 2014, A\&A, 566, A55

Planck Collaboration Int. XIX. 2015, A\&A, 576, A104

Planck Collaboration Int. XX. 2015, A\&A, 576, A105

Planck Collaboration Int. XXIX. 2014, A\&A, submitted [arXiv: 1409.2495]

Planck Collaboration Int. XXX. 2015, A\&A, in press,

DOI: $10.1051 / 0004-6361 / 201425034$ 
Planck HFI Core Team 2011, A\&A, 536, A4

Reach, W. T., Wall, W. F., \& Odegard, N. 1998, ApJ, 507, 507

Rubiño-Martín, J. A., López-Caraballo, C. H., Génova-Santos, R., \& Rebolo, R. 2012, Adv. Astron., 2012, 351836

Rybicki, G. B., \& Lightman, A. P. 1979, Radiative processes in astrophysics (Wiley-Interscience)

Seon, K.-I., \& Witt, A. N. 2012, ApJ, 758, 109

Silsbee, K., Ali-Haïmoud, Y., \& Hirata, C. M. 2011, MNRAS, 411, 2750

Smith, C. H., Wright, C. M., Aitken, D. K., Roche, P. F., \& Hough, J. H. 2000, MNRAS, 312, 327

Tauber, J. A., Mandolesi, N., Puget, J.-L., et al. 2010, A\&A, 520, A1

Vaillancourt, J. E. 2002, ApJS, 142, 53

Vaillancourt, J. E., \& Matthews, B. C. 2012, ApJS, 201, 13

Vaillancourt, J. E., Dowell, C. D., Hildebrand, R. H., et al. 2008, ApJ, 679, L25

Witt, A. N., Gold, B., Barnes, III, F. S., et al. 2010, ApJ, 724, 1551

Wolff, M. J., Clayton, G. C., Kim, S.-H., Martin, P. G., \& Anderson, C. M. 1997 ApJ, 478, 395

Ysard, N., Miville-Deschênes, M. A., \& Verstraete, L. 2010, A\&A, 509, L1

1 APC, AstroParticule et Cosmologie, Université Paris Diderot, CNRS/IN2P3, CEA/lrfu, Observatoire de Paris, Sorbonne Paris Cité, 10 rue Alice Domon et Léonie Duquet, 75205 Paris Cedex 13, France

2 African Institute for Mathematical Sciences, 6-8 Melrose Road, Muizenberg, Cape Town, South Africa

3 Agenzia Spaziale Italiana Science Data Center, via del Politecnico snc, 00133, Roma, Italy

4 Agenzia Spaziale Italiana, Viale Liegi 26 Roma, Italy

5 Astrophysics Group, Cavendish Laboratory, University of Cambridge, J J Thomson Avenue, Cambridge CB3 OHE, UK

6 Astrophysics \& Cosmology Research Unit, School of Mathematics, Statistics \& Computer Science, University of KwaZulu-Natal, Westville Campus, Private Bag X54001, 4000 Durban, South Africa

7 Atacama Large Millimeter/submillimeter Array, ALMA Santiago Central Offices, Alonso de Cordova 3107, Vitacura, Casilla 7630355 Santiago, Chile

8 CITA, University of Toronto, 60 St. George St., Toronto, ON M5S 3H8, Canada

9 CNR - ISTI, Area della Ricerca, via G. Moruzzi 1, Pisa, Italy

10 CNRS, IRAP, 9 Av. colonel Roche, BP 44346, 31028 Toulouse Cedex 4, France

11 California Institute of Technology, Pasadena, California, USA

12 Centro de Estudios de Física del Cosmos de Aragón (CEFCA), Plaza San Juan, 1, planta 2, 44001 Teruel, Spain

13 Computational Cosmology Center, Lawrence Berkeley National Laboratory, Berkeley, California, USA

14 DSM/Irfu/SPP, CEA-Saclay, 91191 Gif-sur-Yvette Cedex, France

15 DTU Space, National Space Institute, Technical University of Denmark, Elektrovej 327, 2800 Kgs. Lyngby, Denmark

16 Département de Physique Théorique, Université de Genève, 24 quai E. Ansermet, 1211 Genève 4, Switzerland

17 Departamento de Física Fundamental, Facultad de Ciencias, Universidad de Salamanca, 37008 Salamanca, Spain

18 Departamento de Física, Universidad de Oviedo, Avda. Calvo Sotelo s/n, Oviedo, Spain

19 Department of Astronomy and Astrophysics, University of Toronto, 50 Saint George Street, Toronto, Ontario, Canada

20 Department of Astrophysics/IMAPP, Radboud University Nijmegen, PO Box 9010, 6500 GL Nijmegen, The Netherlands

21 Department of Physics \& Astronomy, University of British Columbia, 6224 Agricultural Road, Vancouver, British Columbia, Canada

22 Department of Physics and Astronomy, Dana and David Dornsife College of Letter, Arts and Sciences, University of Southern California, Los Angeles, CA 90089, USA

23 Department of Physics and Astronomy, University College London, London WC1E 6BT, UK

24 Department of Physics, Gustaf Hällströmin katu 2a, University of Helsinki, Helsinki, Finland
25 Department of Physics, Princeton University, Princeton, New Jersey, USA

26 Department of Physics, University of California, Santa Barbara, California, USA

27 Department of Physics, University of Illinois at Urbana-Champaign, 1110 West Green Street, Urbana, Illinois, USA

28 Dipartimento di Fisica e Astronomia G. Galilei, Università degli Studi di Padova, via Marzolo 8, 35131 Padova, Italy

29 Dipartimento di Fisica e Scienze della Terra, Università di Ferrara, via Saragat 1, 44122 Ferrara, Italy

30 Dipartimento di Fisica, Università La Sapienza, P. le A. Moro 2, Roma, Italy

31 Dipartimento di Fisica, Università degli Studi di Milano, via Celoria, 16, Milano, Italy

32 Dipartimento di Fisica, Università degli Studi di Trieste, via A. Valerio 2, Trieste, Italy

33 Dipartimento di Fisica, Università di Roma Tor Vergata, via della Ricerca Scientifica, 1 Roma, Italy

34 Discovery Center, Niels Bohr Institute, Blegdamsvej 17, Copenhagen, Denmark

35 Dpto. Astrofísica, Universidad de La Laguna (ULL), 38206 La Laguna, Tenerife, Spain

36 European Southern Observatory, ESO Vitacura, Alonso de Cordova 3107, Vitacura, Casilla 19001 Santiago, Chile

37 European Space Agency, ESAC, Planck Science Office, Camino bajo del Castillo, s/n, Urbanización Villafranca del Castillo, Villanueva de la Cañada, Madrid, Spain

38 European Space Agency, ESTEC, Keplerlaan 1, 2201 AZ Noordwijk, The Netherlands

39 Helsinki Institute of Physics, Gustaf Hällströmin katu 2, University of Helsinki, Helsinki, Finland

40 INAF-Osservatorio Astronomico di Padova, Vicolo dell'Osservatorio 5, Padova, Italy

41 INAF-Osservatorio Astronomico di Roma, via di Frascati 33, Monte Porzio Catone, Italy

42 INAF-Osservatorio Astronomico di Trieste, via G.B. Tiepolo 11, Trieste, Italy

43 INAF/IASF Bologna, via Gobetti 101, Bologna, Italy

44 INAF/IASF Milano, via E. Bassini 15, Milano, Italy

45 INFN, Sezione di Bologna, via Irnerio 46, 40126 Bologna, Italy

46 INFN, Sezione di Roma 1, Università di Roma Sapienza, P.le Aldo Moro 2, 00185, Roma, Italy

47 INFN/National Institute for Nuclear Physics, via Valerio 2, 34127 Trieste, Italy

48 IPAG: Institut de Planétologie et d'Astrophysique de Grenoble, Université Joseph Fourier, Grenoble 1/CNRS-INSU, UMR 5274, 38041 Grenoble, France

49 Imperial College London, Astrophysics group, Blackett Laboratory, Prince Consort Road, London, SW7 2AZ, UK

50 Infrared Processing and Analysis Center, California Institute of Technology, Pasadena, CA 91125, USA

51 Institut d'Astrophysique Spatiale, CNRS (UMR 8617) Université Paris-Sud 11, Bâtiment 121, Orsay, France

52 Institut d'Astrophysique de Paris, CNRS (UMR 7095), 98bis Bd Arago, 75014 Paris, France

53 Institute for Space Sciences, Bucharest-Magurale, Romania

54 Institute of Astronomy, University of Cambridge, Madingley Road, Cambridge CB3 0HA, UK

55 Institute of Theoretical Astrophysics, University of Oslo, Blindern, Oslo, Norway

56 Instituto de Astrofísica de Canarias, C/Vía Láctea s/n, La Laguna, Tenerife, Spain

57 Instituto de Astronomia, Geofísica e Ciências Atmosféricas, Universidade de São Paulo, SP 05508-090 São Paulo, Brazil

58 Instituto de Física de Cantabria (CSIC-Universidad de Cantabria), Avda. de los Castros s/n, Santander, Spain

59 Jet Propulsion Laboratory, California Institute of Technology, 4800 Oak Grove Drive, Pasadena, California, USA 
60 Jodrell Bank Centre for Astrophysics, Alan Turing Building, School of Physics and Astronomy, The University of Manchester, Oxford Road, Manchester, M13 9PL, UK

61 Kavli Institute for Cosmology Cambridge, Madingley Road, Cambridge, CB3 0HA, UK

62 LAL, Université Paris-Sud, CNRS/IN2P3, Orsay, France

63 LERMA, CNRS, Observatoire de Paris, 61 avenue de l'Observatoire, Paris, France

${ }^{64}$ Laboratoire AIM, IRFU/Service d'Astrophysique - CEA/DSM CNRS - Université Paris Diderot, Bât. 709, CEA-Saclay, 91191 Gifsur-Yvette Cedex, France

65 Laboratoire Traitement et Communication de l'Information, CNRS (UMR 5141) and Télécom ParisTech, 46 rue Barrault, 75634 Paris Cedex 13, France

${ }^{66}$ Laboratoire de Physique Subatomique et de Cosmologie, Université Joseph Fourier Grenoble I, CNRS/IN2P3, Institut National Polytechnique de Grenoble, 53 rue des Martyrs, 38026 Grenoble Cedex, France

67 Laboratoire de Physique Théorique, Université Paris-Sud 11 \& CNRS, Bâtiment 210, 91405 Orsay, France

${ }^{68}$ Lawrence Berkeley National Laboratory, Berkeley, California, USA

69 Max-Planck-Institut für Astrophysik, Karl-Schwarzschild-Str. 1, 85741 Garching, Germany

70 National University of Ireland, Department of Experimental Physics, Maynooth, Co. Kildare, Ireland

71 Niels Bohr Institute, Blegdamsvej 17, Copenhagen, Denmark
72 Observational Cosmology, Mail Stop 367-17, California Institute of Technology, Pasadena, CA, 91125, USA

73 Optical Science Laboratory, University College London, Gower Street, London, UK

${ }^{74}$ SISSA, Astrophysics Sector, via Bonomea 265, 34136 Trieste, Italy

75 School of Physics and Astronomy, Cardiff University, Queens Buildings, The Parade, Cardiff, CF24 3AA, UK

76 Space Sciences Laboratory, University of California, Berkeley, California, USA

77 Special Astrophysical Observatory, Russian Academy of Sciences, Nizhnij Arkhyz, Zelenchukskiy region, 369167 KarachaiCherkessian Republic, Russia

78 Sub-Department of Astrophysics, University of Oxford, Keble Road, Oxford OX1 3RH, UK

79 UPMC Univ. Paris 06, UMR 7095, 98bis boulevard Arago, 75014 Paris, France

${ }^{80}$ Université de Toulouse, UPS-OMP, IRAP, 31028 Toulouse Cedex 4, France

81 Universities Space Research Association, Stratospheric Observatory for Infrared Astronomy, MS 232-11, Moffett Field, CA 94035, USA

82 University of Granada, Departamento de Física Teórica y del Cosmos, Facultad de Ciencias, Granada, Spain

${ }^{83}$ University of Granada, Instituto Carlos I de Física Teórica y Computacional, Granada, Spain

84 Warsaw University Observatory, Aleje Ujazdowskie 4, 00-478 Warszawa, Poland 


\section{Appendix A: Derivation of the CC coefficients}

This appendix details how we compute the dust SED using the $\mathrm{CC}$ analysis in Sect. 4.2. For simplicity, we present the simplest case for intensity from the fit with one template.

We minimize the $\chi_{\mathrm{I}}^{2}$ between the data and the $353 \mathrm{GHz}$ template maps, as expressed in Eq. (7). The CC coefficient is then given as

$\left[\alpha_{v}^{\mathrm{I}}\right]_{353}^{1 \mathrm{~T}}=\frac{\sum_{k=1}^{N_{\text {pix }}} \hat{I}_{v}(k) \cdot \hat{I}_{353}(k)}{\sum_{k=1}^{N_{\text {pix }}} \hat{I}_{353}(k)^{2}}$,

where $\hat{I}_{v}$ and $\hat{I}_{353}$ are the data and $353 \mathrm{GHz}$ template with mean values (computed over the $N_{\text {pix }}$ ) subtracted. The observed Planck map at a given frequency is written as the sum of the CMB signal, the Galactic signals (synchrotron, free-free, dust and AME) and noise as

$I_{v}(k)=I^{\mathrm{c}}(k)+I_{v}^{\mathrm{d}}(k)+I_{v}^{\mathrm{s}}(k)+I_{v}^{\mathrm{f}}(k)+I_{v}^{\mathrm{e}}(k)+I_{v}^{\mathrm{n}}(k)$,

where the superscripts $\mathrm{c}, \mathrm{d}, \mathrm{s}, \mathrm{f}, \mathrm{e}$, and $\mathrm{n}$ represent the CMB, dust, synchrotron, free-free, AME, and noise, respectively. Combining Eqs. (A.1) and (A.2) we find

$$
\begin{aligned}
& {\left[\alpha_{v}^{\mathrm{I}}\right]_{353}^{1 \mathrm{~T}}=N \sum_{k=1}^{N_{\text {pix }}}\left[\hat{I}^{\mathrm{c}}(k)+\hat{I}_{v}^{\mathrm{d}}(k)+\hat{I}_{v}^{\mathrm{s}}(k)+\hat{I}_{v}^{\mathrm{f}}(k)+\hat{I}_{v}^{\mathrm{e}}(k)+\hat{I}_{v}^{\mathrm{n}}(k)\right]} \\
& \times\left[\hat{I}^{\mathrm{c}}(k)+\hat{I}_{353}^{\mathrm{d}}(k)+\hat{I}_{353}^{\mathrm{s}}(k)+\hat{I}_{353}^{\mathrm{f}}(k)+\hat{I}_{353}^{\mathrm{e}}(k)+\hat{I}_{353}^{\mathrm{n}}(k)\right],
\end{aligned}
$$

where $N=\left(1 / \sum_{k=1}^{N_{\text {pix }}} \hat{I}_{353}(k)^{2}\right)$ is the normalization factor. At HFI frequencies, we can neglect the contribution of the synchrotron, free-free, AME, and noise within our global mask. This reduces Eq. (A.3) to

$$
\begin{aligned}
{\left[\alpha_{v}^{\mathrm{I}}\right]_{353}^{1 \mathrm{~T}} } & =N \sum_{k=1}^{N_{\text {pix }}}\left(\hat{I}^{\mathrm{c}}(k)+\hat{I}_{v}^{\mathrm{d}}(k)\right) \times\left(\hat{I}^{\mathrm{c}}(k)+\hat{I}_{353}^{\mathrm{d}}(k)\right) \\
& =N \sum_{k=1}^{N_{\text {pix }}}\left[\hat{I}^{\mathrm{c}}(k)\left(\hat{I}^{\mathrm{c}}(k)+\hat{I}_{353}^{\mathrm{d}}(k)\right)+\hat{I}_{v}^{\mathrm{d}}(k)\left(\hat{I}^{\mathrm{c}}(k)+\hat{I}_{353}^{\mathrm{d}}(k)\right)\right] \\
& =\alpha\left(c_{353}\right)+N \sum_{k=1}^{N_{\text {pix }}} \hat{I}_{v}^{\mathrm{d}}(k)\left(\hat{I}^{\mathrm{c}}(k)+\hat{I}_{353}^{\mathrm{d}}(k)\right)
\end{aligned}
$$

The CMB contribution, $\alpha\left(c_{353}\right)$, is the $\mathrm{CC}$ coefficient obtained by correlating the CMB signal map with the $353 \mathrm{GHz}$ template, which is independent of frequency. The dust emission at a given frequency is a scaled version of $353 \mathrm{GHz}$ dust emission, $\hat{I}_{v}^{\mathrm{d}}(k)=$ $\alpha_{v}^{\mathrm{d}} \hat{I}_{353}^{\mathrm{d}}(k)$, where $\alpha_{v}^{\mathrm{d}}$ is the mean dust SED over the given sky patch. The CC coefficient is then

$$
\begin{aligned}
{\left[\alpha_{v}^{\mathrm{I}}\right]_{353}^{1 \mathrm{~T}} } & =\alpha\left(c_{353}\right)+\alpha_{v}^{\mathrm{d}} N \sum_{k=1}^{N_{\mathrm{pix}}} \hat{I}_{353}^{\mathrm{d}}(k)\left(\hat{I}^{\mathrm{c}}(k)+\hat{I}_{353}^{\mathrm{d}}(k)\right) \\
& =\alpha\left(c_{353}\right)+\alpha_{v}^{\mathrm{I}}\left(d_{353}\right) .
\end{aligned}
$$

where $\alpha_{v}^{\mathrm{I}}\left(d_{353}\right)$ is proportional to the mean dust SED. The colour ratio $R_{100}^{\mathrm{I}}(353,217)$ is then given by

$$
R_{100}^{\mathrm{I}}(353,217)=\frac{\left[\alpha_{353}^{\mathrm{I}}\right]_{353}^{1 \mathrm{~T}}-\left[\alpha_{100}^{\mathrm{I}}\right]_{353}^{1 \mathrm{~T}}}{\left[\alpha_{217}^{\mathrm{I}}\right]_{353}^{1 \mathrm{~T}}-\left[\alpha_{100}^{\mathrm{I}}\right]_{353}^{1 \mathrm{~T}}}=\frac{\alpha_{353}^{\mathrm{d}}-\alpha_{100}^{\mathrm{d}}}{\alpha_{217}^{\mathrm{d}}-\alpha_{100}^{\mathrm{d}}} .
$$

In the ratio the scaling between the $\alpha_{v}^{\mathrm{I}}\left(d_{353}\right)$ and $\alpha_{v}^{\mathrm{d}}$ goes away. The colour ratio only depends on the dust spectral properties and not on the CMB signal. The extension of Eq. (A.5) in the presence of AME, synchrotron, and free-free emission at the WMAP and LFI frequencies is given by Eq. (8).

The CMB contribution, $\alpha\left(c_{353}\right)$, in the presence of inverse noise-weighting can be written as

$\alpha\left(c_{353}\right)=\frac{\sum_{k=1}^{N_{\mathrm{pix}}} w_{\nu}(k) \hat{I}^{\mathrm{c}}(k)\left(\hat{I}^{\mathrm{c}}(k)+\hat{I}_{353}^{\mathrm{d}}(k)\right)}{\sum_{k=1}^{N_{\mathrm{pix}}} w_{v}(k) \hat{I}_{353}(k)^{2}}$,

where $w_{v}$ is a weighting factor given by $w_{v}=\left(1 / \sigma_{I_{v}}^{2}\right)$. If the weighting factor depends on the frequency, the CMB contribution is not strictly constant in $\mathrm{K}_{\mathrm{CMB}}$ units. This effect can reach up to $2 \%$, as the weighting factors for the WMAP and Planck maps are quite different. This is not negligible compared to the dust emission at microwave frequencies. That is the reason why we do not use inverse noise-weighting in our $\chi_{\mathrm{I}}^{2}$ minimization.

In Sect. 9.2.1 we compute the frequency dependence of synchrotron emission correlated with dust. The mean spectrum of this component is given by

$$
\begin{aligned}
\left\langle\alpha_{v}^{\mathrm{I}}\left(s_{353}\right)\right\rangle & =N\left\langle\sum_{k=1}^{N_{\text {pix }}} \hat{I}_{v}^{\mathrm{s}}(k)\left(\hat{I}^{\mathrm{c}}(k)+\hat{I}_{353}^{\mathrm{d}}(k)\right)\right\rangle \\
& =N\left\langle\sum_{k=1}^{N_{\text {pix }}} \hat{I}_{v}^{\mathrm{s}}(k) \hat{I}_{353}^{\mathrm{d}}(k)\right\rangle,
\end{aligned}
$$

assuming the CMB chance correlation term with synchrotron emission is zero over all the sky patches. We detect $\left\langle\alpha_{v}^{\mathrm{I}}\left(s_{353}\right)\right\rangle$ with high-significance in our analysis (Sect. 9.2.1), which cannot be just a chance correlation term. One would expect such a correlation, since synchrotron emission arises from the same ISM as dust emission.

Similarly for polarization, we minimize $\chi_{\mathrm{P}}^{2}$ between the data and $353 \mathrm{GHz}$ Stokes $Q$ and $U$ maps, as given by Eq. (13). The polarization $\mathrm{CC}$ coefficient is then given by

$\left[\alpha_{v}^{\mathrm{P}}\right]_{353}^{1 \mathrm{~T}}=N_{\mathrm{P}} \sum_{i=1}^{2} \sum_{k=1}^{N_{\mathrm{pix}}}\left[\hat{Q}_{v}(k) \cdot \hat{Q}_{353}^{i}(k)+\hat{U}_{v}(k) \cdot \hat{U}_{353}^{i}(k)\right]$,

where $N_{\mathrm{P}}$ is the normalization factor for polarization. Following the same logic as described for intensity, the polarization $\mathrm{CC}$ coefficient at the HFI frequencies can be written as

$$
\begin{aligned}
{\left[\alpha_{v}^{\mathrm{P}}\right]_{353}^{1 \mathrm{~T}}=} & \alpha^{\mathrm{P}}\left(c_{353}\right) \\
& +N_{\mathrm{P}} \sum_{i=1}^{2} \sum_{k=1}^{N_{\text {pix }}}\left[\hat{Q}_{v}^{\mathrm{d}}(k)\left(\hat{Q}^{\mathrm{c}}(k)+\hat{Q}_{353}^{\mathrm{d}}(k)+\hat{Q}_{353}^{\mathrm{n}_{\mathrm{i}}}(k)\right)\right] \\
& +N_{\mathrm{P}} \sum_{i=1}^{2} \sum_{k=1}^{N_{\text {pix }}}\left[\hat{U}_{v}^{\mathrm{d}}(k)\left(\hat{U}^{\mathrm{c}}(k)+\hat{U}_{353}^{\mathrm{d}}(k)+\hat{U}_{353}^{\mathrm{n}_{\mathrm{i}}}(k)\right)\right] .
\end{aligned}
$$

Assuming the dust polarization at a given frequency is a scaled version of $353 \mathrm{GHz}$ dust polarization yields $\hat{Q}_{v}^{\mathrm{d}}(k)=\alpha_{v}^{\mathrm{d}} \hat{Q}_{353}^{\mathrm{d}}(k)$ and $\hat{U}_{v}^{\mathrm{d}}(k)=\alpha_{v}^{\mathrm{d}} \hat{U}_{353}^{\mathrm{d}}(k)$. Putting this back to into Eq. (A.10) gives

$\left[\alpha_{v}^{\mathrm{P}}\right]_{353}^{1 \mathrm{~T}}=\alpha^{\mathrm{P}}\left(c_{353}\right)+\alpha_{v}^{\mathrm{P}}\left(d_{353}\right)$.

The polarized colour ratio does not depend on the CMB like Eq. (A.5). The polarized CMB contribution $\alpha^{\mathrm{P}}\left(c_{353}\right)$ is strictly constant in $\mathrm{K}_{\mathrm{CMB}}$ units if we do not apply any noise weighting, 
Planck Collaboration: Frequency dependence of thermal emission from Galactic dust in intensity and polarization

Table B.1. The ancillary data sets and models used in the Monte Carlo simulations.

\begin{tabular}{lcccc}
\hline \hline Components & Tracer & Model & Parameters & References \\
\hline CMB $\ldots \ldots \ldots \ldots$ & TT spectrum & & Planck best-fit parameters & Planck Collaboration XV (2014) \\
Thermal dust $\ldots \ldots \ldots$ & Hi template & MBB & $\left(\beta_{\mathrm{d}}, T_{\mathrm{d}}\right)=(1.5,19.6 \mathrm{~K})$ & Planck Collaboration Int. XVII (2014) \\
IR-HI excess $\ldots \ldots \ldots$ & Residual at $857 \mathrm{GHz}$ & $\mathrm{MBB}$ & $\left(\beta_{\mathrm{R}}, T_{\mathrm{R}}\right)=(2.0,19.6 \mathrm{~K})$ & Planck Collaboration Int. XVII (2014) \\
Free-free $\ldots \ldots \ldots$. & $\mathrm{H} \alpha$ map & $\mathrm{PL}$ & $\left(\beta_{\mathrm{f}}, T_{\mathrm{e}}\right)=(-2.14,7000 \mathrm{~K})$ & Dickinson et al. (2003) \\
CIB $\ldots \ldots \ldots \ldots$ & CIB spectrum & $\mathrm{MBB}$ & $\left(\beta_{\mathrm{CIB}}, T_{\mathrm{CIB}}\right)=(1.3,18.4 \mathrm{~K})$ & Planck Collaboration XXX (2014) \\
Statistical noise $\ldots \ldots$ & Variance maps & & $(I I, I Q, I U, Q Q, Q U, U U)$ & Planck Collaboration VI (2014) \\
Polarized thermal dust & Model & MBB & $\left(\beta_{\mathrm{d}}, T_{\mathrm{d}}\right)=(1.6,19.6 \mathrm{~K})$ & \\
\hline
\end{tabular}

similar to the intensity analysis. To deal with the noise, we first smooth all the maps to $1^{\circ}$ resolution and then perform correlation over local patches on the sky. To compute the uncertainty on the CC coefficients, we rely on Monte Carlo simulations, as discussed in Appendix B.

\section{Appendix B: Simulations}

This appendix presents the simulations of the sky emission in intensity and polarization at HFI frequencies that we use to test the $\mathrm{CC}$ analysis. The intensity and polarization emission components are listed in Table B.1. The simulations use a simplified model of dust emission in intensity and polarization that is good enough to provide a realistic framework to test the $\mathrm{CC}$ analysis. They are computed on HEALPix pixels at $N_{\text {side }}=128$ with a $1^{\circ}$ Gaussian beam. The Monte Carlo simulations serve two specific purposes. First, we use them to check that the CC analysis does not introduce any bias on our estimations of the mean dust spectral indices in intensity and polarization. Second, they provide realistic uncertainties on the $\mathrm{CC}$ coefficients, which we use in the spectral fit to separate out the dust and the CMB emission (Sect. 5.3).

\section{B.1. Intensity}

At HFI frequencies, the main diffuse emission components are the thermal dust, free-free, CMB, and CIB emission. The simulations also include instrumental noise. We now describe how we simulate each of these components.

The HI column density from the LAB survey (Kalberla et al. 2005) is taken as a proxy for thermal dust emission. We normalize the HI data to a suitable amplitude to match the observed Planck data at $353 \mathrm{GHz}$ and extrapolate to the other HFI frequencies using an MBB spectrum with a fixed spectral index $\beta_{\mathrm{d}}=1.5$ and temperature $T_{\mathrm{d}}=19.6 \mathrm{~K}$ over the whole sky. The HI data provide only a partial description of the thermal dust emission, as quoted in Planck Collaboration Int. XVII (2014). We include an additional dust component, spatially uncorrelated with the HI data, to mimic the residuals present after adopting the IR-HI correlation at $857 \mathrm{GHz}$. The additional dust-like emission is assumed to have an $\ell^{-3}$ power spectrum, with a normalized amplitude of $4 \pi \sigma_{857}^{2}$ for $\ell=2$, where $\sigma_{857}$ is the residual at $857 \mathrm{GHz}$ after applying the IR-HI correlation and removing the CIB contribution (Planck Collaboration Int. XVII 2014). The amplitude of the uncorrelated HI emission is normalized at $857 \mathrm{GHz}$, taken from Planck Collaboration Int. XVII (2014), and scaled to the HFI frequencies assuming $\beta_{\mathrm{R}}=2.0$ for a dust temperature of $T_{\mathrm{R}}=19.6 \mathrm{~K}$. We use the DDD $\mathrm{H} \alpha$ map as a proxy for freefree emission, which we compute at HFI frequencies for a spectral index $\beta_{\mathrm{f}}=-2.14$ (in $\mathrm{K}_{\mathrm{RJ}}$ units; Planck Collaboration Int. XIV 2014) and an electron temperature $T_{\mathrm{e}}=7000 \mathrm{~K}$ (Dickinson et al. 2003). No dust extinction correction is applied to the DDD $\mathrm{H} \alpha$ map.
For the $\mathrm{CMB}$, we compute Gaussian realizations of the CMB sky from the theoretical power spectrum of the Planck best-fit model (Planck Collaboration XV 2014). The CIB emission is generated using the best-fit model of CIB anisotropies at $353 \mathrm{GHz}$ obtained directly from the Planck data (Planck Collaboration XXX 2014). We assume $100 \%$ correlated CIB across all the HFI frequencies, assuming an MBB spectrum with $\beta_{\mathrm{CIB}}=1.3$ and $T_{\mathrm{CIB}}=18.4 \mathrm{~K}$. The Gaussian realizations of the instrumental noise are obtained at each frequency, using the noise variance maps (Planck Collaboration VI 2014). The noise realizations are simulated at the full resolution of the Planck data, before smoothing to $1^{\circ}$ resolution and reducing the pixelization from $N_{\text {side }}=2048$ to 128 .

We compute 1000 realizations of sky maps of the additional dust component, together with the CMB and CIB anisotropies. Independent realizations of the instrumental noise are generated for each sky simulation at a given frequency. The dust component computed from the HI map and the free-free emission traced by the $\mathrm{H} \alpha$ map are kept fixed.

We analyse the 1000 simulated maps with the CC method applied to the Planck intensity data. We compute the mean and standard deviation of the $R_{100}^{\mathrm{I}}(353,217)$ values for each sky patch. Both are plotted in Fig. B.1 versus the local dispersion of the $353 \mathrm{GHz}$ template, $\sigma_{353}^{\mathrm{I}}$. There is no bias on the estimation of the mean dust spectral index $\beta_{\mathrm{d}, \mathrm{mm}}^{\mathrm{I}}$. We recover a mean value equal to the index of 1.5 we used for the main HI-correlated dust component. The uncertainties on $R_{100}^{\mathrm{I}}(353,217)$, and hence on $\beta_{\mathrm{d}, \mathrm{mm}}^{\mathrm{I}}$, are associated with noise, CIB anisotropies, free-free emission, and the additional dust component. The $1 \sigma$ dispersion of $\beta_{\mathrm{d}, \mathrm{mm}}^{\mathrm{I}}$ across sky patches for a given Monte Carlo realization is 0.02. This is smaller than the scatter of 0.07 measured for the Planck data. We interpret the difference as evidence for a small intrinsic dispersion in the spectral index of the dust emission.

\section{B.2. Polarization}

The simulations of the polarized sky at HFI frequencies include polarized CMB, thermal dust emission, and noise. We compute 1000 realizations of the CMB Stokes $Q$ and $U$ maps using the best-fit Planck model (Planck Collaboration XV 2014), smoothed to $1^{\circ}$ resolution at HEALPix resolution $N_{\text {side }}=128$. Random realizations of Gaussian noise $Q$ and $U$ maps are generated at each pixel using the $3 \times 3$ noise covariance matrix defined at $N_{\text {side }}=2048$. The noise maps are then smoothed to $1^{\circ}$ resolution and projected on to a HEALPix map at $N_{\text {side }}=128$. We generate independent realizations of the instrumental noise to mimic the detector sets at $353 \mathrm{GHz}$ (Sect. 2.1.2).

For polarized thermal dust emission, we use the following model:

$\left[Q_{v}^{\mathrm{d}}, U_{v}^{\mathrm{d}}\right]=p_{\mathrm{d}}\left(\frac{v}{v_{\text {ref }}}\right)^{\beta_{\mathrm{d}, \mathrm{mm}}^{\mathrm{P}}} \frac{B_{v}\left(T_{\mathrm{d}}\right)}{B_{v_{\text {ref }}}\left(T_{\mathrm{d}}\right)} I_{v_{\text {ref }}}^{\mathrm{d}}\left[\cos 2 \psi_{\mathrm{d}}, \sin 2 \psi_{\mathrm{d}}\right]$. 

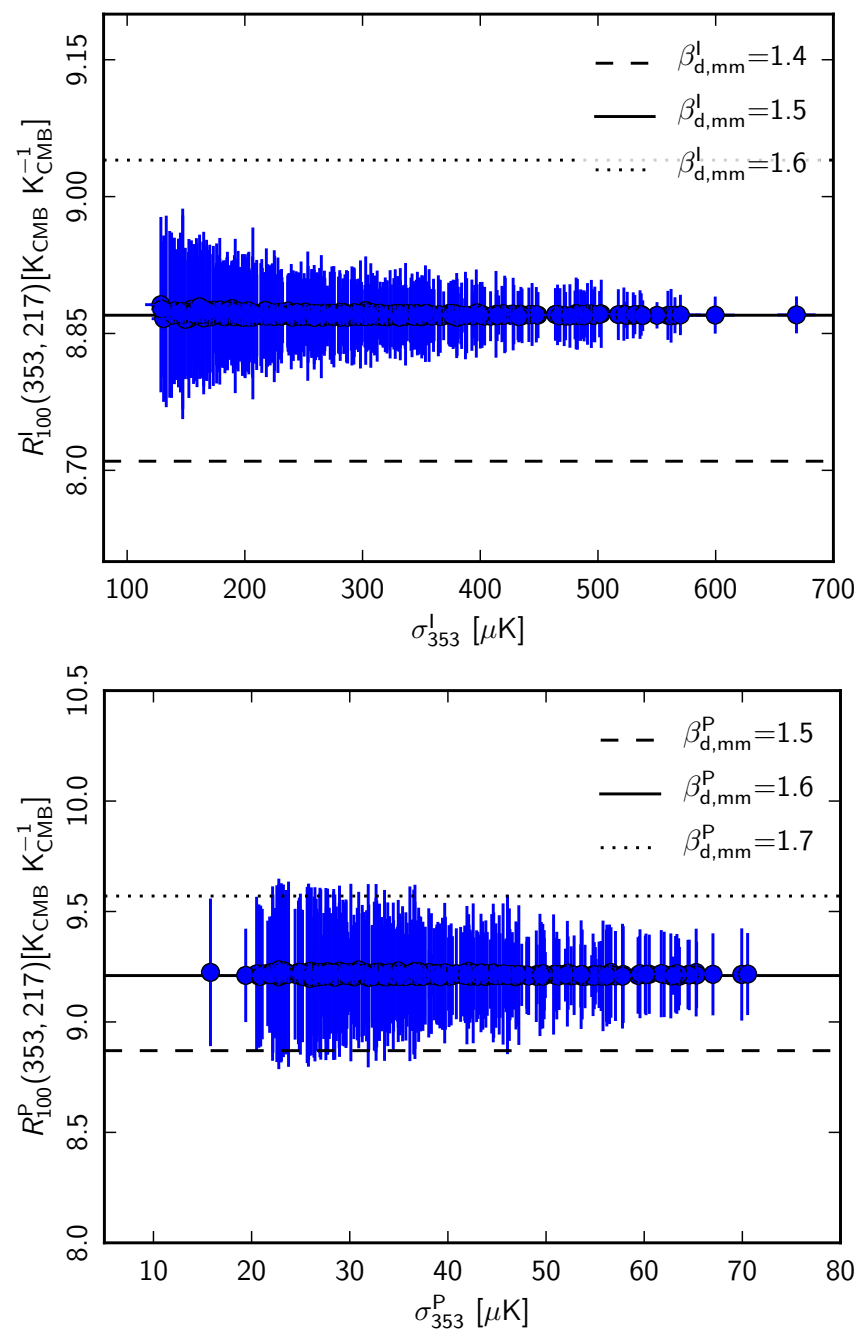

Fig. B.1. $R_{100}(353,217)$ colour ratios from the Monte Carlo simulations for intensity (top) and polarization (bottom). The two plots show that the $\mathrm{CC}$ analysis does not introduce any bias on the estimation of $\beta_{\mathrm{d}, \mathrm{mm}}$.

Here $p_{\mathrm{d}}, I_{\text {ref }}^{\mathrm{d}}$, and $\psi_{\mathrm{d}}$ are the polarization fraction, the dust intensity at reference frequency, and the polarization angle, respectively. The reference frequency is $v_{\text {ref }}=353 \mathrm{GHz}$. We fix $p_{\mathrm{d}}$ to a constant value of $10 \%$ over the whole sky. The $I_{\text {ref }}^{\mathrm{d}}$ map is that obtained by Planck Collaboration XI (2014) from the spectral fit to the high frequency Planck and IRAS $100 \mu \mathrm{m}$ data, with an MBB model. For the dust polarization, we use an MBB spectrum with $\beta_{\mathrm{d}, \mathrm{mm}}^{\mathrm{P}}=1.6$ and $T_{\mathrm{d}}=19.6 \mathrm{~K}$, constant over the whole sky. We derive $\psi_{\mathrm{d}}$ from the $1^{\circ}$ smoothed Planck Stokes maps using the relation

$\psi_{\mathrm{d}}=-0.5 \times \operatorname{atan} 2\left(U_{v_{2}}^{\mathrm{obs}}-U_{v_{1}}^{\mathrm{obs}}, Q_{v_{2}}^{\mathrm{obs}}-Q_{v_{1}}^{\mathrm{obs}}\right)$.

We choose $v_{1}$ and $v_{2}$ as the 143 and $353 \mathrm{GHz}$, respectively. The difference between the two frequencies removes the CMB contribution.

We analyse 1000 polarized simulated maps using the $\mathrm{CC}$ analysis as applied to the Planck data. We compute the mean and the standard deviation of the $R_{100}^{\mathrm{P}}(353,217)$ for each sky patch. The plot of $R_{100}^{\mathrm{P}}(353,217)$ versus $\sigma_{353}^{\mathrm{P}}$ is shown in Fig. B.1. We find no bias in the estimation of $R_{100}^{\mathrm{P}}(353,217)$ and hence in the measurement of $\beta_{\mathrm{d}, \mathrm{mm}}^{\mathrm{P}}$. The $1 \sigma$ dispersion of $\beta_{\mathrm{d}, \mathrm{mm}}^{\mathrm{P}}$ across sky patches for a given simulation is 0.07 . The $1 \sigma$ dispersion of $\beta_{\mathrm{d}, \mathrm{mm}}^{\mathrm{P}}$ from the simulations is smaller compared than that measured from the Planck data, because we use a simplified

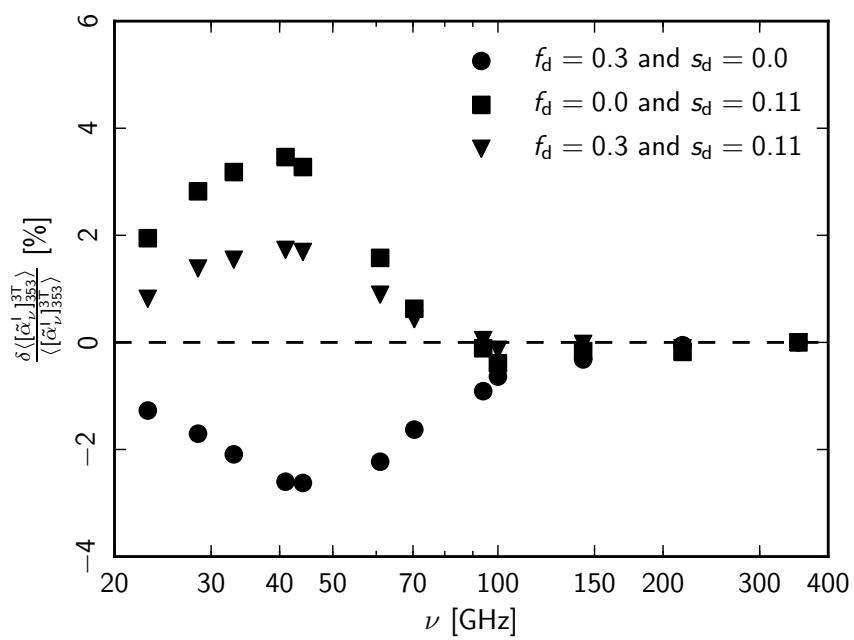

Fig. C.1. Fractional change in the mean dust SED with respect to the reference dust SED presented in this paper, for different combinations of $f_{\mathrm{d}}$ and $s_{\mathrm{d}}$ corrections on the DDD $\mathrm{H} \alpha$ template.

white noise model. However, some of the dispersion may come from the intrinsic dispersion of the polarized dust spectral index, and also additional Galactic polarized emission components, which we neglect in the simulations.

\section{Appendix C: Mean dust SED with dust extinction and scattering correction on the $\mathrm{H} \alpha$ template}

The mean dust SED for intensity presented in this paper is obtained using the three-template fit with no extinction and dust scattering correction from the DDD $\mathrm{H} \alpha$ template. The effect of dust extinction $\left(f_{\mathrm{d}}\right)$ on the $\mathrm{H} \alpha$ template is described in Eq. (3) of Dickinson et al. (2003), whereas the effect of dust scattering $\left(s_{\mathrm{d}}\right)$ on the $\mathrm{H} \alpha$ template is described in Eq. (26) of Bennett et al. (2013). The mean measured value of $s_{\mathrm{d}}$ is $0.11 \mathrm{R}\left(\mathrm{MJy} \mathrm{sr}^{-1}\right)^{-1}$ in high Galactic latitude regions (Lehtinen et al. 2010; Witt et al. 2010; Seon \& Witt 2012; Brandt \& Draine 2012; Bennett et al. 2013). To check the impact of the $f_{\mathrm{d}}$ and $s_{\mathrm{d}}$ corrected $\mathrm{H} \alpha$ template on the mean dust SED, we repeat the analysis with different combinations of $f_{\mathrm{d}}$ and $s_{\mathrm{d}}$. The three different combinations of $f_{\mathrm{d}}$ and $s_{\mathrm{d}}$ corrected $\mathrm{H} \alpha$ templates we choose are: $f_{\mathrm{d}}=0.3$ and $s_{\mathrm{d}}=0.0 \mathrm{R}\left(\mathrm{MJy} \mathrm{sr}^{-1}\right)^{-1} ; f_{\mathrm{d}}=0.0$ and $s_{\mathrm{d}}=0.11 \mathrm{R}\left(\mathrm{MJy} \mathrm{sr}^{-1}\right)^{-1}$; and $f_{\mathrm{d}}=0.3$ and $s_{\mathrm{d}}=0.11 \mathrm{R}\left(\mathrm{MJy} \mathrm{sr}^{-1}\right)^{-1}$. The fractional change in the mean dust SED with respect to the reference dust $\operatorname{SED}\left(f_{\mathrm{d}}=0.0\right.$ and $\left.s_{\mathrm{d}}=0.0 \mathrm{R}\left(\mathrm{MJy} \mathrm{sr}^{-1}\right)^{-1}\right)$ is presented in Fig. C.1. At higher frequencies $(v \geq 100 \mathrm{GHz})$, the impact of both dust extinction and scattering is negligible. However at frequencies $v \leq 50 \mathrm{GHz}$, the fractional change on the mean SED can go as high as $\pm 4 \%$. The $f_{\mathrm{d}}$ and $s_{\mathrm{d}}$ parameters are degenerate, although their effect on the derived best-fit parameter of models $\mathrm{DI}+\mathrm{AI}$ and DI+AII, listed in Table 4 , is very small.

\section{Appendix D: Power spectra of the templates}

In this section, we compute the temperature power spectra of the three templates at $1^{\circ}$ resolution: $408 \mathrm{MHz}$; DDD $\mathrm{H} \alpha$; and $353 \mathrm{GHz}$ dust template. The Planck $353 \mathrm{GHz}$ map contains a significant component of CMB anisotropies. Taking the SMICA map (Planck Collaboration XII 2014) as a proxy for the CMB map, we remove its contribution from the $353 \mathrm{GHz}$ total map. These spectra are combined with the SEDs from this paper to compute the contributions of each emission component to the microwave sky emission as a function of angular scales in Planck Collaboration I (2014, see their Figs. 27 and 28). 
Planck Collaboration: Frequency dependence of thermal emission from Galactic dust in intensity and polarization
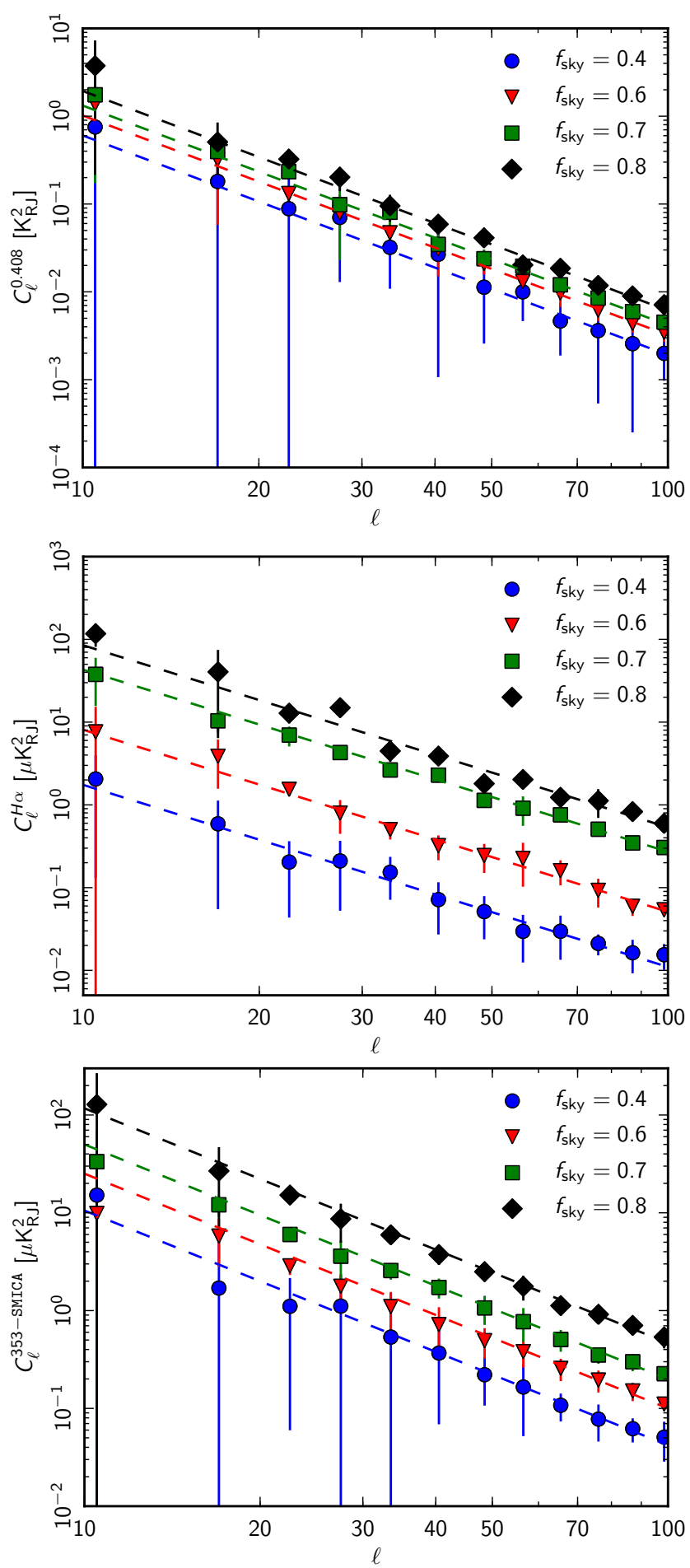

Fig. D.1. Power spectra of the $408 \mathrm{MHz}$, DDD $\mathrm{H} \alpha$, and SMICAsubtracted $353 \mathrm{GHz}$ templates, smoothed to $1^{\circ}$ resolution for different Galactic masks (or $f_{\text {sky }}$ ).

For the computation of the power spectra, we consider the four diffuse Galactic masks based on the percentage of the sky retained $\left(f_{\text {sky }}\right)$, i.e., G40, G60, G70 and G80 (Planck Collaboration XV 2014). The same set of masks have been used in the likelihood analysis of the 2013 Planck data release (Planck Collaboration XV 2014). The power spectra are computed only at low multipoles $(\ell<100)$ with PolSpice v2.9.0 (Chon et al. 2004), corrected for the masking, beam, and pixel window effect. Figure D. 1 presents binned power spectra of the three templates: $408 \mathrm{MHz}$, DDD H $\alpha$, and SMICA-subtracted $353 \mathrm{GHz}$ maps as a
Table D.1. Amplitudes of the power spectra, normalized at $\ell=100$, as a function of $f_{\text {sky }}$.

\begin{tabular}{lcccc}
\hline \hline Gal. & $f_{\text {sky }}$ & $\begin{array}{c}A_{0.408} \\
{\left[10^{9} \mu \mathrm{K}_{\mathrm{RJ}}^{2}\right]} \\
\text { masks }\end{array}$ & $\begin{array}{c}A_{\mathrm{H} \alpha} \\
{[\mu=-2.5)}\end{array}$ & $\begin{array}{c}\left.A_{353-\text { SMICA }}^{2}\right] \\
{\left[\mu \mathrm{K}_{\mathrm{RJ}}^{2}\right]} \\
(\alpha=-2.2)\end{array}$ \\
\hline G40 & 0.40 & $1.913 \pm 0.492$ & $0.011 \pm 0.002$ & $0.042 \pm 0.007$ \\
G60 & 0.60 & $3.223 \pm 0.314$ & $0.051 \pm 0.003$ & $0.101 \pm 0.009$ \\
G70 & 0.70 & $4.187 \pm 0.379$ & $0.271 \pm 0.016$ & $0.199 \pm 0.016$ \\
G80 & 0.80 & $6.139 \pm 0.489$ & $0.536 \pm 0.026$ & $0.466 \pm 0.029$ \\
\hline
\end{tabular}

function of the Galactic masks. The uncertainties on the binned power spectra include only the statistical variance and not the cosmic variance.

At low multipoles, $\ell<100$, the three power spectra are wellfit with a power-law model. Using this assumption, the measured power spectra are written as $C_{\ell}=A \times(\ell / 100)^{\alpha}$. Here $A$ represents the normalized amplitude at $\ell=100$ and $\alpha$ represents the slope of the power-law for a given template. We fix $\alpha$ based on the measured spectra and only fit for the amplitudes as a function of the Galactic masks. We find that the slope of the $408 \mathrm{MHz}$ spectra over all the Galactic masks is consistent with -2.5 . In case of DDD $\mathrm{H} \alpha$ template is -2.2 over the masks and the same for the SMICA-subtracted $353 \mathrm{GHz}$ template is -2.4 . The results of the power-law fit for the three templates and different Galactic masks are shown as a dashed lines in Fig. D.1. The amplitudes of each of the templates as a function of the Galactic masks (or $f_{\text {sky }}$ ) are listed in Table D.1.

The amplitudes of the given templates vary nonlinearly as

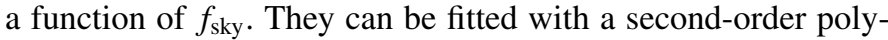
nomial in a $\log A-\log f_{\text {sky }}$ plane. Combining the $v, \ell$, and $f_{\text {sky }}$ dependence, we analytically model the power spectra of the diffuse synchrotron, free-free, and dust emission components for intensity. For amplitude normalization, we made an assumption on the nature of the synchrotron and free-free emission. We assume a single power-law model for the synchrotron emission from $408 \mathrm{MHz}$ to microwave frequencies, $v \leq 353 \mathrm{GHz}$. For free-free emission, we assume a single power-law model at microwave frequencies, with a mean electron temperature of $7000 \mathrm{~K}$ (Dickinson et al. 2003). The power spectra of the three diffuse emission components, in $\mu \mathrm{K}_{\mathrm{RJ}}^{2}$ units, are

$$
\begin{aligned}
C_{\ell}^{\mathrm{f}}= & 0.068 \times\left(\frac{f_{\text {sky }}}{0.6}\right)^{\left[6.10+3.90 \ln \left(f_{\text {sky }} / 0.6\right)\right]} \\
& \times\left(\frac{\ell}{100}\right)^{-2.2} \times\left(\frac{v}{v_{\mathrm{b}}}\right)^{-4.28},
\end{aligned}
$$

$$
\begin{aligned}
C_{\ell}^{\mathrm{s}}= & 2.96 \times 10^{9} \times\left(\frac{f_{\text {sky }}}{0.6}\right)^{\left[2.12+2.67 \ln \left(f_{\text {sky }} / 0.6\right)\right]} \\
& \times\left(\frac{\ell}{100}\right)^{-2.5} \times\left(\frac{v}{v_{\mathrm{c}}}\right)^{-6.0},
\end{aligned}
$$

$C_{\ell}^{\mathrm{d}}=0.086 \times\left(\frac{f_{\text {sky }}}{0.6}\right)^{\left[4.60+7.11 \ln \left(f_{\text {sky }} / 0.6\right)\right]} \times\left(\frac{\ell}{100}\right)^{-2.4} \times \mathcal{D}_{v}$

where $v_{\mathrm{b}}=23 \mathrm{GHz}, v_{\mathrm{c}}=0.408 \mathrm{GHz}, \mathcal{D}_{v}$ is a spectral model of the dust emission given by one of the two models presented in Eqs. (26), and (27). The derived analytical model of these power spectra are valid in the frequency range 20 to $353 \mathrm{GHz}$, and for $f_{\text {sky }}$ between 0.4 and 0.8 . 